\title{
Two-phase reheating: CMB constraints on inflation and dark matter phenomenology
}

\author{
Md Riajul Haque, ${ }^{1, *}$ Debaprasad Maity, ${ }^{1, \dagger}$ and Pankaj Saha ${ }^{2, \$}$ \\ ${ }^{1}$ Department of Physics, Indian Institute of Technology Guwahati, Guwahati, Assam 781039, India \\ ${ }^{2}$ Department of Physics, Indian Institute of Technology Madras, Chennai, Tamilnadu 600036, India
}

(Received 16 September 2020; accepted 21 September 2020; published 26 October 2020)

\begin{abstract}
We propose a two-phase reheating scenario where the initial preheating dynamics is described by an effective dynamics followed by the standard perturbative reheating. Some of the important universal results of lattice simulation during preheating have been considered as crucial inputs in our two-phase dynamics. In this framework, detailed phenomenological constraints have been obtained on the inflaton couplings with reheating fields and dark matter parameters in terms of the cosmic microwave background (CMB) constrained inflationary scalar spectral index. It is observed that the conventional reheating scenario generically predicts the maximum reheating temperature $T_{\mathrm{re}}^{\max } \simeq 10^{15} \mathrm{GeV}$, corresponding to an almost instantaneous transition from the end of inflation to radiation domination. This fact will naturally lead to the problem of nonperturbative inflaton decay, which is in direct conflict with the perturbative reheating itself. Taking into account this by incorporating effective nonperturbative dynamics as the initial phase, our model of two-phase reheating scenarios also predicts a model-independent maximum reheating temperature, which does not correspond to the instantaneous process. Furthermore, $T_{\mathrm{re}}^{\max }$ is predicted to lie within $\left(10^{13}, 10^{10}\right) \mathrm{GeV}$ if $\mathrm{CMB}$ constraints on inflaton couplings with a different reheating field are taken into account. We have further studied in detail the dark matter phenomenology in a model-independent manner and show how dark matter parameter space can be constrained through CMB parameters via the inflaton spectral index. Considering dark matter production during reheating via the freeze-in mechanism, its parameter space has been observed to be highly constrained by our two-phase reheating than the constraints predicted by the conventional reheating scenarios, which are believed to theoretically incomplete.
\end{abstract}

DOI: 10.1103/PhysRevD.102.083534

\section{INTRODUCTION}

The inflationary universe $[1,2]$ is currently the leading paradigm to explain the inhomogeneities in the cosmic microwave background (CMB) [3], which plays the crucial role of seed perturbations for the large-scale structure of the Universe [4]. Within the present setup, the inflationary phase must be followed by a phase known as reheating ${ }^{1}$ when the energy stored in the inflaton field is released to defrost the Universe [7-10]. Unlike inflation, the reheating phase is not constrained by direct observables; however, the modified expansion history of the Universe due to the

riaju176121018@iitg.ac.in

†debu@iitg.ac.in

pankaj@physics.iitm.ac.in

${ }^{1}$ Particle production during inflation is also considered in the so-called warm inflationary scenario $[5,6]$.

Published by the American Physical Society under the terms of the Creative Commons Attribution 4.0 International license. Further distribution of this work must maintain attribution to the author(s) and the published article's title, journal citation, and DOI. Funded by SCOAP ${ }^{3}$. presence of the reheating phase prior to the hot big bang evolution influences the relation between physical scales of the CMB mode today and that at the time of their Hubble exit during inflation (see Fig. 2 in this context). This was the basic idea of reheating constraints to inflationary models from the CMB [11]. Despite the thermalization process erasing many microphysical details of this phase, a better understanding of this phase is necessary and can shed light on how the inflationary mechanism is connected to the rest of the Universe dynamics [12-14], the production mechanism of baryonic asymmetry in the early Universe called baryogenesis [15-21], the origin of dark matter [22], and the generation of primordial gravitational waves [23-33] and constraining them, etc. There exist two approaches that can constrain the reheating phase through the inflationary models. Either we can model the expansion during the reheating phase using an effective equation of state parameter [11,34], or we can solve the Boltzmann equation system supplemented with the background expansion [35]. Both descriptions have their limitations and are not theoretically complete. However, the latter approach's advantage is that one can further generalize it by including matter components in addition to radiation, which could be 
physically motivated. For example, in the original work, the production of dark matter has been studied. The study revealed a very interesting link between the dark matter with CMB through inflation [35]. Later, this formalism has been extended in considering various models of inflation with a general power-law-type potential [36], and the nonperturbative effect from numerical lattice simulation has also been considered [37]. In this paper, we take up this issue of the nonperturbative phase known as preheating and formulate an effective approach that will be shown to lead to qualitatively different results than that of the usual reheating constraint analysis. Preheating is the phase when the occupation number of field quanta for both the inflaton and daughter field(s) grows exponentially due to parametric resonance [38]. The equation of state during the steady state of the preheating phase is crucial for model-independent reheating constraint analysis. Furthermore, some scenarios can lead to a nonstandard case such as a sudden blocking reheating process due to the Higgs field [39] or a breakdown of coherent oscillation without thermalization [40]. Considering those into our present scenario would be interesting to consider. Although the preheating phenomenon depends on the inflation model and its interaction with the daughter fields, certain universal behaviors have been observed to emerge irrespective of inflation models $[37,41,42]$. Namely, (i) the preheating phase is episodic with at least three distinct phases. (iii) The equation of state (EOS) of the system does not reach to that of the radiation for quadratic potentials $V(\phi) \propto \phi^{2}$. However, for another form $V(\phi) \propto \phi^{n}$ with $n \geq 4$, the EOS reaches $w \rightarrow 1 / 3$ at the end of preheating [37,42-44].

These results indicate that, while nonperturbative processes dominate the initial stage, the inflaton decay in later stages should be described by perturbative channels. A systematic study of reheating constraints incorporating the nonperturbative phase for various models and interactions is missing in the literature.

In this work, we will extend the formalism developed in Refs. [35,37] to include the nonperturbative effects in reheating constraint analysis. As we just mentioned, reheating happens in multiple stages, with the initial nonperturbative stage followed by the perturbative one. Let us now briefly describe the main idea of the present work: Endowed with the above few universal features, we will consider reheating as a two-stage process. We will model the initial nonperturbative stage (henceforth, phase I) governed by an effective fluid with an effective equation of state $\left(w_{\text {eff }}\right)$. One of the boundary conditions of the effective nonperturbative dynamics will be set by the inflation model potential expressed in terms of the scalar spectral index (in this regard, the reader may find this in parallel with the conventional works in Ref. [11]). This phase is assumed to be continued until the inflaton energy decaying into $50 \%$ of its initial energy and the subsequent perturbative stage (henceforth, phase II) follows. While evolving through phase I and connecting to phase II, we allow the system to satisfy an important consistency relation associated with the total energy conservation comprising inflaton and various daughter fields such as radiation and dark matter (which we considered separately). We will see that this consistency relation will restrict the possible values of $w_{\text {eff }}$ during phase I as opposed to the conventional analysis [11]. Furthermore, the perturbative decay in phase II will be restricted by the CMB constraints [35].

We have structured our paper as follows: In Sec. II, we discuss the general analysis of single-stage perturbative reheating, and in Sec. III, we will try to specify the possible limits on perturbative reheating considering some specified form of the interaction between inflation and radiation fields. Finally, in Sec. IV, we briefly describe our proposed two-phase reheating analysis and, in Sec. V, illustrate the strategy of our numerical study. After that, in Sec. VI, we will try to find out an analytical estimation of the maximum radiation temperature and reheating temperature. Next, in Sec. VII, we consider different inflationary models and analyze in the context of the two-phase scenario and compare it with conventional reheating dynamics. In Sec. VIII, we analyze the possible constraints on the coupling parameter corresponding to different inflatonradiation field interactions. Furthermore, in Sec. IX, we include additional dark matter components and discuss the viable restrictions on the dark matter parameter space.

\section{REHEATING CONSTRAINT ANALYSIS FOR PERTURBATIVELY DECAYING INFLATON}

Before we directly jump into constructing the two-phase reheating model, let us first elaborate on widely studied single-phase perturbative reheating with decaying inflaton following Ref. [35]. This not only explains the methodology of our analysis, but also helps us to identify the regime of its validity which will further motivate the reader, the need for considering a physically more acceptable twophase reheating process mentioned in the introduction. While discussing this, we will see one of the important results, that is, the existence of a maximum reheating temperature. Subsequently, the generalization to two-phase reheating will show how the aforesaid maximum reheating temperature reduces depending upon the initial condition. Let us start with the following Einstein equation for the cosmological scale factor and conservation of energy:

$$
\begin{aligned}
& \ddot{n}_{\mathrm{re}}=-2 \dot{n}_{\mathrm{re}}^{2}+\frac{1-3 w}{6 M_{p}^{2}} \rho_{\phi}, \\
& \dot{\rho}_{\phi}+3 \dot{n}_{\mathrm{re}}\left(\rho_{\phi}+p_{\phi}\right)+\dot{\rho}_{\mathrm{rad}}+4 \dot{n}_{\mathrm{re}} \rho_{\mathrm{rad}}=0,
\end{aligned}
$$

with the following Friedmann-Robertson-Walker (FRW) form of the metric:

$$
d s^{2}=-d t^{2}+a^{2}\left(d x^{2}+d y^{2}+d z^{2}\right),
$$


where $\rho$ 's are the energy densities of two different components. At any instant of time during reheating, we parametrize the duration of reheating by $e$-folding number $n_{\mathrm{re}}(t)=\ln \left(a / a_{i}\right)$, where $a$ is the cosmological scale factor. The time derivative of $n_{\mathrm{re}}$ is the Hubble expansion parameter $\dot{n}_{\mathrm{re}}=H$ during reheating. During reheating, we assume the effective equation of state of the inflaton $w=\left\langle p_{\phi} / \rho_{\phi}\right\rangle$ to be approximately constant. The fundamental difference between our present analysis followed from Ref. [35] and that in Ref. [11] is the consideration of Eq. (1), where we consider the multiple dynamical components. Considering the evolution of $\rho_{\phi}+\rho_{\text {rad }}=\rho_{\text {eff }}$ together, the effective equation of state during reheating can be defined as

$$
w_{\text {eff }}=\left\langle\frac{3 p_{\phi}+\rho_{\mathrm{rad}}}{3\left(\rho_{\phi}+\rho_{\mathrm{rad}}\right)}\right\rangle .
$$

Hence, $w_{\text {eff }}$ will essentially interpolates between two values $(w, 1 / 3)$ through nontrivial time dynamics for decaying inflaton $\rho_{\phi}$ and the growing radiation field $\rho_{\text {rad }}$. However, in Ref. [11], the authors have taken it to be constant during their analysis. Therefore, we not only employ realistic decay dynamics into the reheating constraint analysis but also provide a new framework to go beyond, which is our main purpose of the present paper.

Keeping the above points in mind, let us express the total energy density as

$\rho_{\mathrm{rad}}+\rho_{\phi}=e^{-4 n_{\mathrm{re}}}\left(\rho_{\phi}^{i}+(1-3 w) \int_{t_{i}}^{t} \rho_{\phi} e^{4 n_{\mathrm{re}}} d n_{\mathrm{re}}\right)$,

which is followed from the conservation Eq. (1). The index $i$ stands for the initial stage of reheating, which also marks the end of inflation. At the beginning of reheating we set $\rho_{\text {rad }}\left(t_{i}\right)=0$. For solving the above set of equations, the boundary condition is set by the inflaton energy density as $\dot{n}_{\mathrm{re}}\left(t_{i}\right)=H\left(t_{i}\right)=\sqrt{\rho_{\phi}^{i} / 3 M_{p}^{2}}$. The physical quantity of our interest is the ratio of the radiation energy density and the inflaton energy density. From Eq. (4), one gets

$\frac{\rho_{\mathrm{rad}}^{f}}{\rho_{\phi}^{i}}=e^{-4 N_{\mathrm{re}}}-\frac{\rho_{\phi}^{f}}{\rho_{\phi}^{i}}+(1-3 w) e^{-4 N_{\mathrm{re}}} \int_{i}^{f} \frac{\rho_{\phi}}{\rho_{\phi}^{i}} e^{4 n_{r}} d n_{\mathrm{re}}$,

where $f$ corresponds to the final value of radiation density. We define the total $e$-folding number during reheating as $N_{\text {re }}=n_{\text {re }}\left(t_{f}\right)$.

The main goal of this whole program of reheating constraint analysis is to understand the relation among early Universe inflaton dynamics, the intermediate reheating dynamics, and late time dynamics. A particular cosmological scale $k$ going out of the horizon during inflation will reenter the horizon during late time cosmological evolution. This fact will provide an important relation among different phases just mentioned as follows:

$$
\ln \left(\frac{a_{k} H_{k}}{a_{0} H_{0}}\right)=-N_{k}-N_{\mathrm{re}}-\ln \left(\frac{a_{\mathrm{re}} H_{k}}{a_{0} H_{0}}\right)
$$

where a particular scale $k$ satisfies the relation $k=a_{0} H_{0}=a_{k} H_{k} .\left(a_{\mathrm{re}}, a_{0}\right)$ are the cosmological scale factors at the end of reheating phase and at the present time, respectively. $\left(N_{k}, H_{k}\right)$ are the $e$-folding number and the Hubble parameter, respectively, during inflation. $H_{0}$ is the present value of the Hubble constant.

The usual approach is to define the effective equation of state of the total energy density during reheating and study its evolution. However, we consider only the radiation part during reheating and try to understand the evolution of its temperature $T_{\text {rad }}$ as a function of the scalar spectral index and finally connect the temperature with CMB one on the large scale [35]. The reheating temperature $T_{\text {re }}$ is identified with radiation temperature $T_{\text {rad }}$ at thermal equilibrium between the decaying inflaton and the radiation. From the entropy conservation of thermal radiation, the relation among $T_{\text {rad }}=$ $T_{\text {re }}$ at equilibrium, and $\left(T_{0}, T_{\nu 0}=(4 / 11)^{1 / 3} T_{0}\right)$, the temperature of the CMB photon and neutrino background at the present day, respectively, can be written as

$$
g_{\mathrm{re}} T_{\mathrm{re}}^{3}=\left(\frac{a_{0}}{a_{\mathrm{re}}}\right)^{3}\left(2 T_{0}^{3}+6 \frac{7}{8} T_{\nu 0}^{3}\right) .
$$

Using Eqs. (6) and (7), one arrives at the following wellknown relation:

$T_{\mathrm{re}}=\left(\frac{43}{11 g_{\mathrm{re}}}\right)^{1 / 3}\left(\frac{a_{0} T_{0}}{k}\right) H_{k} e^{-N_{k}} e^{-N_{\mathrm{re}}}=\mathcal{G}_{k} e^{-N_{\mathrm{re}}}$,

where $g_{\mathrm{re}} \sim 100$ is the effective number of relativistic degrees of freedom during the radiation phase. In our subsequent study, we identify the cosmological scale $k$ as the pivot scale set by Planck, $k / a_{0}=0.05 \mathrm{Mpc}^{-1}$, and compare our result with the corresponding estimated scalar spectral index $n_{s}=0.9682 \pm 0.0062$ [45].

\section{A. Example I: Exactly solvable case}

As has already been discussed in Ref. [35], one of the important outcomes of our formalism is the existence of a maximum possible reheating temperature. In this and the next section, we will elaborate on this considering a simple ansatz of decaying inflaton. We first consider an analytically solvable case where the inflaton is decaying as

$$
\begin{gathered}
\dot{\rho}_{\phi}+3 H(1+w) \rho_{\phi}=-\bar{\Gamma}_{\phi} H \rho_{\phi} \\
\Rightarrow \rho_{\phi}(t)=\rho_{\phi}^{i} e^{-3(1+w) n_{\mathrm{re}}} e^{-\bar{\Gamma}_{\phi} n_{\mathrm{re}}}
\end{gathered}
$$

$\bar{\Gamma}_{\phi}$ is a dimensionless constant, which parametrizes the decay of inflaton. This form of decay essentially modifies 
the Hubble friction term for the dynamics of inflaton during reheating. With the aforementioned ansatz for the decaying inflaton, the radiation density is analytically solved as

$$
\frac{\rho_{\mathrm{rad}}^{f}}{\rho_{\phi}^{i}}=\frac{\bar{\Gamma}_{\phi}}{\bar{\Gamma}_{\phi}+3 w-1}\left(e^{-4 N_{\mathrm{re}}}-e^{-3(1+w) N_{\mathrm{re}}-\bar{\Gamma}_{\phi} N_{\mathrm{re}}}\right) .
$$

The second term in the parentheses is quantifying the fractional amount of inflaton energy left after the reheating process is over. Expressing $\rho_{f}$ in term of the radiation temperature as $\rho_{\mathrm{rad}}^{f}=\pi^{2}\left(g_{\mathrm{re}} / 30\right) T_{\mathrm{rad}}^{4}$, Eq. (10) leads to the following maximum radiation temperature [46] for a given $\Gamma_{\phi}$ :

$T_{\mathrm{rad}}^{\max }=\left(\frac{30 \rho_{\phi}^{i} P}{\pi^{2} g_{\mathrm{re}}}\right)^{1 / 4}\left[x^{4 /\left(\bar{\Gamma}_{\phi}+3 w-1\right)}-x^{\left(3+3 w+\bar{\Gamma}_{\phi} /\left(\bar{\Gamma}_{\phi}+3 w-1\right)\right.}\right]^{1 / 4}$,

where $\left(x=4 /\left(3+3 w+\bar{\Gamma}_{\phi}\right)\right.$ and $P=\bar{\Gamma}_{\phi} /\left(\bar{\Gamma}_{\phi}+3 w-1\right)$. This also can be clearly seen from Fig. 1 for each value of $\bar{\Gamma}_{\phi}$. From the perturbative point of view, the value of $\Gamma_{\phi}$ should be $\leq 1$. However, if we naively extrapolate the above result for large $\bar{\Gamma}_{\phi}$, the most important result turned out to be the existence of a maximum possible temperature:

$$
\lim _{\bar{\Gamma}_{\phi} \gg 1} T_{\mathrm{rad}}^{\max }=\left(\frac{30 \rho_{\phi}^{i}}{\pi^{2} g_{\mathrm{re}}}\right)^{1 / 4} \simeq 2.9 \times 10^{15} \mathrm{GeV} .
$$

However, the numerical value of this maximum temperature turns out to be of the order of the same as the limiting perturbative value for $\bar{\Gamma}_{\phi}=1$ as shown in Fig. 1. Therefore, the above temperature can be naturally identified as the maximum possible reheating temperature. This also corresponds to the maximum possible value of scalar spectra index $n_{s}^{\max }$. Identifying the associated temperature of the produced radiation in Eq. (10) with Eq. (8), we arrive at the following exact expression for $\left(N_{\text {re }}, T_{\text {re }}\right)$ :

$$
\begin{array}{r}
T_{\mathrm{re}}=\mathcal{G}_{k}\left(1-\frac{1}{P} \frac{\pi^{2} g_{\mathrm{re}} \mathcal{G}_{k}^{4} \cdot{ }^{2} \cdot 5 V_{\mathrm{end}}}{1 /\left[4-3(1+w)-\bar{\Gamma}_{\phi}\right]},\right. \\
N_{\mathrm{re}}=\frac{1}{4-3(1+w)-\bar{\Gamma}_{\phi}} \ln \left[1-\frac{1}{P} \frac{\pi^{2} g_{\mathrm{re}} \mathcal{G}_{k}^{4}}{3^{2} .5 V_{\mathrm{end}}}\right] .
\end{array}
$$

In Fig. 1, we have considered three possible values of $\bar{\Gamma}_{\phi}$ for the quadratic inflaton potential. The special value is $\bar{\Gamma}_{\phi}=1$, for which the equilibrium condition between the inflaton and the radiation can be achieved at the maximum temperature shown as a black dot. The maximum value of the scalar spectral index turned out to be $n_{s}^{\max } \simeq 0.9654$. This analysis motivates us to subsequently analyze a more general case, and we will show that this conclusion still holds.

\section{B. Example II: Standard perturbative case}

In this section, we will consider the standard perturbatively decaying inflaton parameterizing by decay constant $\Gamma_{\phi}$ as follows:

$$
\begin{aligned}
\dot{\rho}_{\phi}+3 H(1+w) \rho_{\phi} & =-\Gamma_{\phi} \rho_{\phi}(1+w) \\
\Rightarrow \rho_{\phi}(t) & =\rho_{\phi}^{i} e^{-3(1+w) n_{\mathrm{re}}} e^{-\Gamma_{\phi}\left(t-t_{i}\right)(1+w)},
\end{aligned}
$$

where $\Gamma_{\phi}$ is the effective time-independent inflaton decay constant. It is the phenomenological term which acts as a damping force during the oscillating inflaton. This term can be related to the total decay rate of inflaton to radiation. However, we believe our conclusion will remain the same for time-dependent $\Gamma_{\phi}$, which we will study later. Before
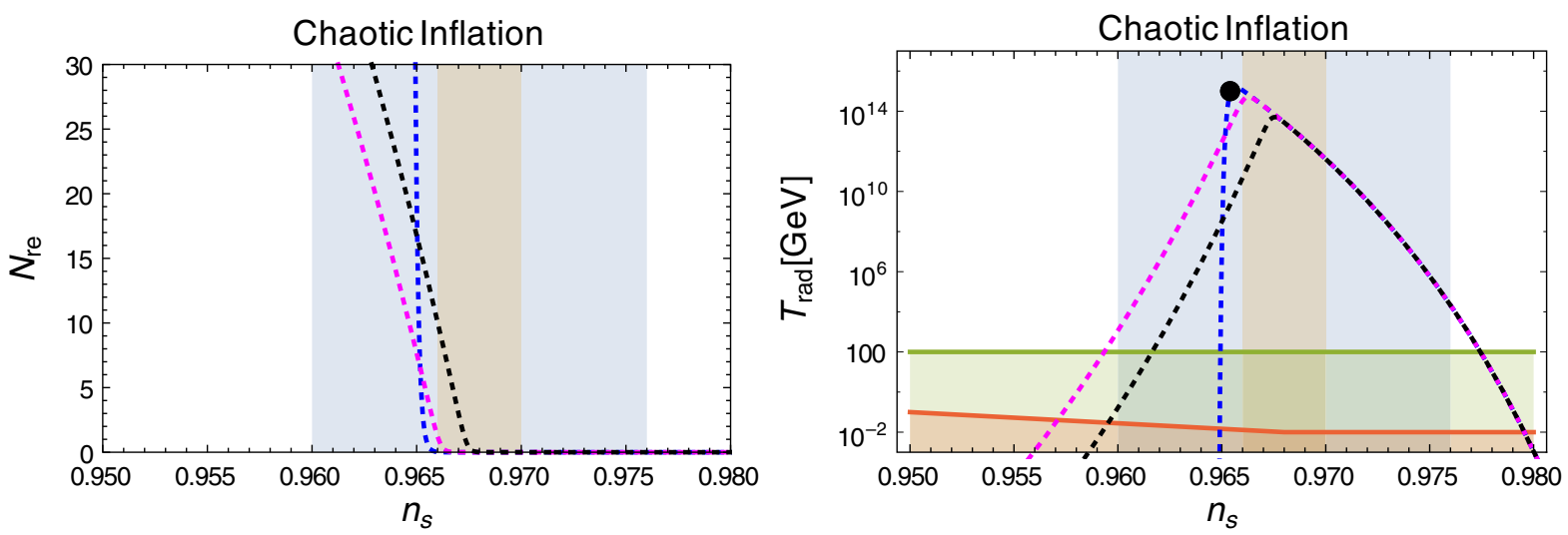

FIG. 1. Variations of $\left(N_{\text {re }}, T_{\text {rad }}\right)$ as a function of $n_{s}$ have been plotted for $\bar{\Gamma}_{\phi}=(1.0,0.01,0.000001)$ corresponding to blue, pink, and black curves, respectively. The light blue shaded region corresponds to the $1 \sigma$ bounds on $n_{s}$ from Planck. The brown shaded region corresponds to the $1 \sigma$ bounds of a further CMB experiment with sensitivity $\pm 10^{-3}[47,48]$, using the same central $n_{s}$ value as Planck. Temperatures below the horizontal red line is ruled out by big bang nucleosynthesis (BBN). The deep green shaded region is below the electroweak scale, assumed $100 \mathrm{GeV}$ for reference. 
doing any numerical analysis, let us examine the approximate solution which has already been discussed in the literature [46]. During the early stage of evolution, approximating $\rho_{\phi}^{i} e^{-\Gamma_{\phi} t} \simeq \rho_{\phi}^{i}$, the radiation density can be calculated as

$$
\frac{\rho_{\mathrm{rad}}^{f}}{\rho_{\phi}^{i}} \simeq \frac{2 \Gamma_{\phi} e^{-4 N_{\mathrm{re}}}}{(5-3 w) \dot{n}_{\mathrm{re}}\left(t_{i}\right)}\left(e^{[(5-3 w) / 2] N_{\mathrm{re}}}-1\right) .
$$

Similar to the exactly solvable case in Eq. (11), the above equation also leads to a maximum radiation temperature [46] for a given $\Gamma_{\phi}$ :

$$
T_{\mathrm{rad}}^{\max } \simeq\left(\frac{39^{2} M_{p}^{2}\left(\dot{n}_{\mathrm{re}}^{i}\right)^{2}}{\pi^{2} g_{\mathrm{re}}}\right)^{1 / 8} \sqrt{T_{\mathrm{re}}},
$$

where the relation $T_{\mathrm{re}}=0.45\left(200 / g_{\mathrm{re}}\right)^{1 / 4} \sqrt{\Gamma_{\phi} M_{p}}$ has been used. In the same way as our earlier exactly solvable case, the maximum possible reheating temperature could be obtained, if one identifies a special point where two temperatures meet, $T_{\text {rad }}^{\max }=T_{\text {re }}$. Our numerical analysis also shows the maximum reheating temperature at the aforementioned special point:

$$
T_{\mathrm{re}}^{\max } \simeq\left(\frac{39^{2} \rho_{\phi}^{i}}{3 \pi^{2} g_{\mathrm{re}}}\right)^{1 / 4} .
$$

Interestingly, the maximum reheating temperature $T_{\mathrm{re}}^{\max }$ can also be computed for another exactly solvable case with $w=1 / 3$. Corresponding result is as follows:

$$
\begin{aligned}
& \left.T_{\mathrm{rad}}^{\max }\right|_{w=1 / 3} \simeq\left(\frac{30 \rho_{\phi}^{i}}{\pi^{2} g_{\mathrm{re}}} \frac{\Gamma_{\phi}}{4 \dot{n}_{i}+\Gamma_{\phi}}\right)^{1 / 4}, \\
& \left.T_{\mathrm{re}}^{\max }\right|_{w=1 / 3}=\left.\lim _{\Gamma_{\phi} \gg 4 \dot{n}_{i}} T_{\mathrm{rad}}^{\max }\right|_{w=1 / 3}=\left(\frac{30 \rho_{\phi}^{i}}{\pi^{2} g_{\mathrm{re}}}\right)^{1 / 4} .
\end{aligned}
$$

This expression is exactly the same as previously discussed. For this special value of $w=1 / 3$, we also have the exact expression for all the reheating parameters $\left(T_{\text {re }}, N_{\text {re }}\right)$ as follows:

$$
\begin{gathered}
T_{\mathrm{re}}=\mathcal{G}_{k}\left(1-\sqrt{\frac{4 \rho_{\phi}^{i}}{3 M_{p}^{2} \Gamma_{\phi}^{2}}} \ln \left[1-\frac{\pi^{2} g_{\mathrm{re}} \mathcal{G}_{k}^{4}}{3^{2} \cdot 5 V_{\mathrm{end}}}\right]\right)^{-1 / 2}, \\
N_{\mathrm{re}}=\frac{1}{2} \ln \left[1-\sqrt{\frac{4 \rho_{\phi}^{i}}{3 M_{p}^{2} \Gamma_{\phi}^{2}}} \ln \left[1-\frac{\pi^{2} g_{\mathrm{re}} \mathcal{G}_{k}^{4}}{3^{2} \cdot 5 V_{\mathrm{end}}}\right]\right] .
\end{gathered}
$$

At this point, let us again emphasize the fact that, as long as we are in the perturbative regime, the relation among the scalar spectral index $n_{s}$ and the reheating temperature $T_{\text {re }}$ can be understood from our detail analysis above. However, the existence of a maximum reheating temperature will come if we extrapolate all our formulas for large $\Gamma_{\phi}>\sqrt{2 \rho_{i} /\left(3 M_{p}^{2}\right)}$. For low-scale inflation, $\Gamma_{\phi}$ could always be in the perturbative regime. For large-scale inflation, this could lead to the nonperturbative regime, which will be discussed in our subsequent section. We will discuss possible limits on the value of $\Gamma_{\phi}$ below which our analysis will be valid. To this end, it is important to point out that, in the effective reheating equation of state description [11], the maximum temperature can be explained in the limit of zero reheating $e$-folding number $N_{\text {re }}$. Therefore, the large $\Gamma_{\phi}$ limit in our analysis can be thought of as equivalent to the zero $N_{\text {re }}$ limit of the previously studied reheating constraint analysis. However, it is important to remember that those two facts are certainly not identical. Corresponding to our maximum temperature, we have a minimum reheating $e$-folding number. Our prediction of maximum reheating temperature $\sim 10^{15} \mathrm{GeV}$ and its model independence could be robust, and they are intimately connected with the observed CMB scale.

Nevertheless, the main point of our study is to understand the effect of decaying inflaton into the reheating constraint analysis. We think this is the appropriate procedure to understand the relation among $\left(T_{\mathrm{re}}, n_{s}\right)$. Another advantage of our procedure is that we can easily generalize our analysis to include any other decay products during reheating such as dark matter, which is observed to be a dominant matter component of our Universe [49-52], and that can shape the observed pattern in the CMB. Before this, our main motivation would be to incorporate the nonperturbative aspects of reheating into our formalism.

\section{REGIME OF VALIDITY OF PERTURBATIVE REHEATING}

In this section, we will try to mention the possible limits on the inflaton decay constant assuming some specific form of the interactions among the inflaton and the reheating field. As emphasized throughout the present work, we have assumed that the inflation decay to other components (for the present work, the radiation component) is effectively described by a phenomenological decay term $\Gamma_{\phi}$. In fact, this was the first attempt to reheat the Universe [53]. However, it was soon realized that, once the particle production initiates, the inflaton decay is subject to various nonperturbative resonance production and feedback mechanisms. Those processes can change the reheating scenario dramatically, though it has been argued in Ref. [12] that all such feedback mechanisms will have no effect on the CMB. Depending upon the coupling, the parametric resonance can be very efficient, which may complete the reheating era within a few $e$-foldings, and in such cases the CMB will have very little to tell about the reheating phase. Despite that, the situation may not be such helpless as noted in Ref. [54,55] that the interactions among the produced particles can delay the parametric resonance extending 
the $e$-folding number of reheating. This will eventually improve the situation of the $\mathrm{CMB}$ constraint on the reheating phase. It must also be noted that we can always choose the coupling constant small enough to evade the parametric resonance. Below, we will briefly mention the space of parameter region in which the perturbative treatment of reheating will be valid over the parametric resonance.

\section{A. Inflaton decaying into scalar particle}

\section{Scalar $\phi \chi^{2}$ interaction}

First, let us consider the case when inflaton decays into another scalar particle $\phi \rightarrow \chi \chi$ with the following interaction term $\mathcal{L}=-g \phi \chi^{2}$. In this case, the vacuum decay width for the decay process $\phi \rightarrow \chi \chi$ is given by [56]

$$
\Gamma_{\phi \rightarrow \chi \chi}=\frac{g^{2}}{8 \pi m_{\phi}} \sqrt{1-\left(\frac{2 m_{\chi}}{m_{\phi}}\right)^{2}} \simeq \frac{g^{2}}{8 \pi m_{\phi}},
$$

where $m_{\phi}$ and $m_{\chi}$ are the mass of the inflaton and produced particle, respectively, and $g$ is the coupling constant. The mode function $\chi_{k}$ of the decay product can be cast into the following Mathieu equation:

$$
\ddot{\chi_{k}}+\left(A_{k}-2 q \cos (2 z)\right) \chi_{k}=0,
$$

where $\quad z=\left(m_{\phi} t-2 z-\pi / 2\right), \quad A_{k}=4 k^{2} / m_{\phi}^{2}, \quad$ and $q=4 g \Phi / m_{\phi}^{2} . \Phi$ is the initial amplitude of the inflaton during oscillations. The Mathieu equation is known to show resonance solutions of the form $\chi_{k} \propto \exp \left(\mu_{k} z\right)$. The condition for the resonance to be efficient is formulated as

$$
q^{2} m \gtrsim H .
$$

This can be transformed into the following condition ${ }^{2}$ on the dimensionless coupling constant $\tilde{g}=g / m_{\phi}$ indicating the regime of perturbative validity [57]:

$$
\tilde{g} \leq \frac{V_{\text {end }}^{1 / 4}}{\phi_{\text {end }}}\left(\frac{m_{\phi}}{24 M_{p}}\right)^{1 / 2},
$$

which can further expressed in terms of the decay constant as

$$
\Gamma_{\phi} \leq \frac{V_{\text {end }}^{1 / 2}}{\phi_{\text {end }}^{2}}\left(\frac{m_{\phi}^{2}}{192 \pi M_{p}}\right) \Rightarrow \Gamma_{\phi}^{\mathrm{cri}}(\operatorname{model})=\frac{V_{\text {end }}^{1 / 2}}{\phi_{\text {end }}^{2}}\left(\frac{m_{\phi}^{2}}{192 \pi M_{p}}\right) .
$$

Therefore, we see that if the decay width satisfies the aforementioned condition, the perturbative reheating will

\footnotetext{
${ }^{2}$ In deriving this condition, the initial amplitude $\Phi$ has been replaced by $\phi_{\text {end }}$, which implies that this is essentially a lower bound on the decay width as in the case of preheating $\Phi<\phi_{\text {end }}$.
}

be the only mechanism and our perturbative analysis will be at work. Given a model $\Gamma_{\phi}^{\text {cri }}($ model $)$ is the point which qualitatively separates the perturbative and nonperturbative effects of inflaton decay.

\section{Scalar $\phi \chi^{3}$ interaction}

In this case, inflaton couples to another light scalar via interaction

$$
\mathcal{L}=-y \phi \chi^{3},
$$

where $y$ is the coupling constant. The vacuum decay rate of the inflaton field into three bodies $\phi \rightarrow \chi \chi \chi$ can be determined by a Dalitz plot [58] as

$$
\Gamma_{\phi \rightarrow \chi x \chi}=\frac{y^{2} m_{\phi}}{3 ! 64(2 \pi)^{3}} .
$$

At the tree level, the mode function $\chi_{k}$ following the same Mathieu equation and the consideration from the previous scalar $\phi \chi^{2}$ interaction can be correlated if one replaces $\tilde{g} m_{\phi} \Phi \rightarrow h^{2} \Phi^{2}$. Therefore, the condition to treat the dynamics of reheating perturbatively is roughly

$$
q \backsim \frac{y^{2} \Phi^{2}}{m_{\phi}^{2}} \leq 1
$$

To estimate the lower bound on the coupling for the resonance, we make a substitution $\Phi \rightarrow \phi_{\text {end }}$. The above condition for the effectiveness of perturbative reheating can be written in terms of the decay rate as

$\Gamma_{\phi} \leq \frac{m_{\phi}^{3}}{3 ! 64(2 \pi)^{3} \phi_{\text {end }}^{2}} \Rightarrow \Gamma_{\phi}^{\text {cri }}($ model $)=\frac{m_{\phi}^{3}}{3 ! 64(2 \pi)^{3} \phi_{\text {end }}^{2}}$.

Thereafter, in our analysis, we want to examine whether this above condition is consistent with our analysis or not for the different inflationary models.

\section{B. Inflaton decaying into a pair of fermions}

Let us now consider the case when the inflaton decays into a pair of massless fermions with the following Yukawa interaction:

$$
\mathcal{L}_{\text {int }}=-h \phi \bar{\psi} \psi,
$$

where $h$ is the dimensionless coupling constant. Now the vacuum decay rate is given by

$$
\Gamma_{\phi \rightarrow \bar{\psi} \psi}=\frac{h^{2} m_{\phi}}{8 \pi} .
$$


The condition for the validity of perturbative reheating in this case, as shown in Ref. [59], can be written as

$$
q=\frac{h^{2} \Phi^{2}}{m_{\phi}^{2}} \leq 1
$$

Hence, in connection with the decay rate, Eq. (33) is rewritten as

$$
\Gamma_{\phi} \leq \frac{m_{\phi}^{3}}{\phi_{\text {end }}^{2}(8 \pi)} \Rightarrow \Gamma_{\phi}^{\mathrm{cri}}(\text { model })=\frac{m_{\phi}^{3}}{\phi_{\mathrm{end}}^{2}(8 \pi)}
$$

In our proposed effective two-phase dynamical scenario, we will observe the existence of a similar critical inflaton decay constant associated with the reheating $e$-folding number. We will see how the aforementioned three different interacting model-dependent critical decay constants restrict in initial parameter space of the reheating dynamics. In the following sections, our attempt will be to build up a formalism which can effectively incorporate the nonperturbative dynamics at the initial stage of the reheating.

\section{REGIME OF EFFECTIVE NONPERTURBATIVE AND PERTURBATIVE REHEATING}

The standard and well-studied mechanism to consider the nonperturbative effect during reheating is called preheating. This stage is essentially the combination of a highly nonlinear process of parametric resonance and subsequent thermalization. It is a well-known fact that a generically nonperturbative preheating mechanism does not completely decay inflaton into the radiation field. Therefore, subsequent perturbative decay will be necessary to complete the reheating process. To the best of our knowledge, Ref. [35] has considered this issue for the first time and studied perturbative reheating, followed by the preheating considering a specific model of chaotic-type inflation. However, generically, the preheating mechanism is model dependent. Hence, combining the end of preheating and subsequent model-independent perturbative reheating is somewhat irreconcilable. Therefore, our objective in the following sections would be to make these two phases reconcilable.

Instead of dwelling on explicit nonperturbative computation during the preheating stage, we will adopt an effective model-independent approach following Ref. [11]. The basic idea is to assume the dynamics of preheating to be solely governed by an effective equation of state $\omega_{\text {eff }}$ supplemented with the total energy conservation law in terms of its constituents. As already emphasized in the introduction, the information about the actual nonperturbative dynamic will be encoded through considering its universal features into our effective dynamics.
As has been pointed out already, during the nonperturbative dynamics, inflaton decay is not complete, and typically it is around $50 \%$ of its total comoving energy, which is being transferred into the daughter fields. Furthermore, for inflation models with a quadratic potential near the minimum, the nonperturbative reheating does not lead to the equation of state, $\omega=\frac{1}{3}$, which is expected at the end point of reheating $[37,60]$. Our essential idea would be to correctly utilize those results as the end point conditions of our proposed effective dynamics in place of preheating. After the end of this, the usual Boltzmann perturbative reheating process will follow. The second phase completes the reheating process by leading to the correct state equation with relativistic degrees of freedom as the dominant components collectively called radiation. Figure 2 illustrates our methodology of calculation. Throughout this paper, we call this the two-phase reheating process.

\section{A. Phase I: (Effective nonperturbative phase)}

During the early stage of reheating, the phase will be described by total energy density $\rho_{T}=\rho_{R}+\rho_{\phi}$ and the constant effective equation state $w_{\text {eff }}$. Hence, the evolution will be described by

$$
\rho_{T}=\rho_{T e}\left(\frac{a_{\mathrm{end}}}{a}\right)^{3\left(1+w_{\mathrm{eff}}\right)}
$$

where $\rho_{T e}$ is the total energy density at the end of the inflation. $a_{\text {end }}$ is the scale factor at the end of the inflation. In this section, we will build up our formalism considering two matter components with $\rho_{\phi}$ and $\rho_{R}$ as the inflaton energy density and radiation energy density, respectively, at any instant of time. In the subsequent section, we will add dark matter as a third component as an extension. Nonetheless, from the total energy expression one can write down the following equation that follows from Eq. (35):

$$
\dot{\rho_{\phi}}+\dot{\rho_{R}}+3 H\left(1+w_{\text {eff }}\right)\left(\rho_{\phi}+\rho_{R}\right)=0 .
$$

To reduce the number of unknown parameters, to this end we will also utilize total energy conservation relation considering the individual equation of state of the inflaton $\left(\omega_{\phi}\right)$ and the radiation field $\left(\omega_{R}=1 / 3\right)$ described as

$$
\dot{\rho_{\phi}}+3 H\left(1+w_{\phi}\right) \rho_{\phi}+\dot{\rho_{R}}+4 H \rho_{R}=0
$$

Given the aforementioned constraint relation, one obtains the possible restricted value of the effective equation of state, $\omega_{\text {eff }}$. To find those restrictions, we combine the above two Eqs. (36) and (37) and obtain the following consistency relation: 


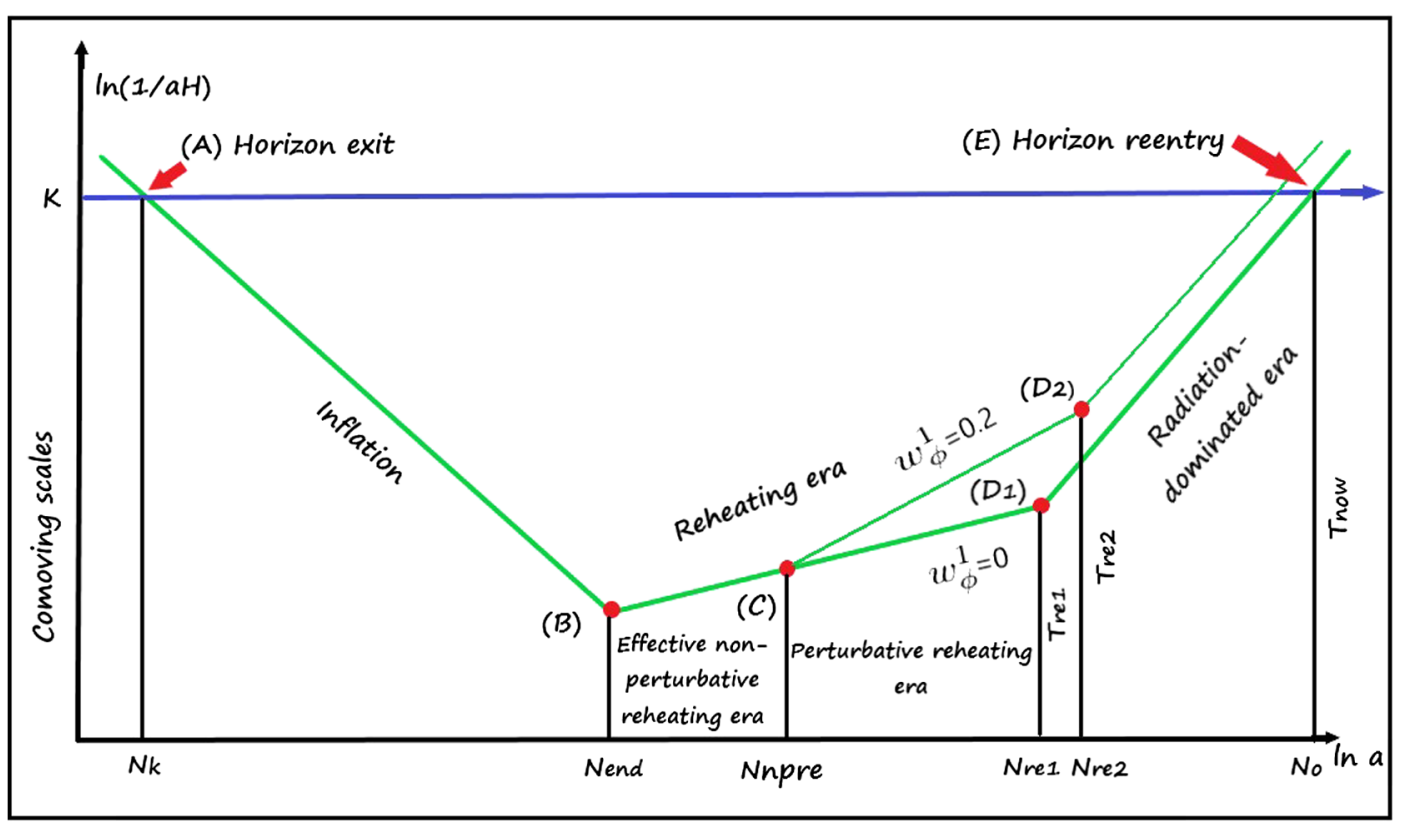

FIG. 2. The evolution of the comoving Hubble scale $\left(\frac{1}{a H}\right)$ connects the inflationary phase with the CMB. The end of the inflation is denoted by point $B$, and the ending of the radiation-dominated era is denoted by the points $D_{1}$ and $D_{2}$. The inflationary phase and radiation-dominated era connect through the reheating phase, which contains two different regions, the effective nonperturbative reheating era and the perturbative reheating era. $C$ denotes the ending point of the nonperturbative reheating era. The points $D_{1}$ and $D_{2}$ are the ending point of the perturbative reheating era for two different inflaton equations of state during perturbative reheating $\omega_{\phi}^{1}=(0,0.2)$, respectively. For the perturbative reheating era with the inflaton equation of state $\omega_{\phi}^{1}=(0,0.2)$, the $e$-folding numbers, basically, the duration of the perturbative process, are different. For a particular value of the spectral index (lower values of $n_{s}$, toward $\left.n_{s}^{\min }\right)$, the decay width calculating by considering $\omega_{\phi}^{1}=0.2$ is quite lower in comparison with $\omega_{\phi}^{1}=0$. That is why, for that particular values of $n_{s}$, the duration of the perturbative era is quite wider for $\omega_{\phi}^{1}=0.2$ in connection with $\omega_{\phi}^{1}=0\left(N_{\text {re2 }}>N_{r e 1}\right)$.

$$
\frac{\rho_{R}}{\rho_{\phi}+\rho_{R}}=\frac{3\left(w_{\phi}-w_{\mathrm{eff}}\right)}{3 w_{\phi}-1} .
$$

Right at the end of inflation or the beginning of the preheating phase, the energy density of the radiation part will naturally be close to zero $\rho_{R} \simeq 0$. As time evolves, $\rho_{R}$ increases due to decaying inflaton. This initial condition automatically restricts the possible values of $w_{\text {eff }}$ to be very close to that of the inflaton equation of state $w_{\phi}$. Of course, a more appropriate approach would be to assume the inflaton equation of state evolving from the value very close to $\omega_{\phi}$ to the value required in the next phase. We will comment on this issue at the appropriate place during our discussion. However, from the usual numerical lattice simulation, the preheating phase's significant duration is dominated by the inflaton. Hence, the equation of state will naturally be close to that of the inflaton field. Thus, our effective dynamics approach toward preheating truly captures all these necessary properties of the nonperturbative dynamics. As $w_{\text {eff }}$ turned out to be no longer a free parameter and constrained by the above consistency relation (38), our following analysis will be based on this important result. Throughout our study, we consider models for which the inflaton equation of state during phase I $\omega_{\phi}=0$, and, consequently, following Eq. (38) we choose two values of effective equation of state $\omega_{\text {eff }}=$ $\left(10^{-6}, 10^{-3}\right)$. This choice will automatically fix the initial radiation densities during phase I as $\rho_{R} /\left(\rho_{\phi}+\rho_{R}\right)=$ $\left(3 \times 10^{-6}, 3 \times 10^{-3}\right)$. We will see that the maximum reheating temperature crucially depends upon these initial conditions.

\section{B. Phase II: (Perturbative phase)}

Once the preheating dynamics ends, the usual Boltzmann perturbative reheating follows. During this period, various components of the total energy density satisfy the following standard Boltzmann equations [61]:

$$
\begin{gathered}
\dot{\rho}_{\phi}+3 H\left(1+w_{\phi}^{1}\right) \rho_{\phi}+\Gamma_{\phi} \rho_{\phi}\left(1+w_{\phi}^{1}\right)=0, \\
\dot{\rho}_{R}+4 H \rho_{R}-\Gamma_{\phi} \rho_{\phi}\left(1+w_{\phi}^{1}\right)=0,
\end{gathered}
$$

where the inflaton field $\phi$ decays into radiation with the decay rate $\Gamma_{\phi} . \omega_{\phi}^{1}$ represents the inflaton equation of state during perturbative reheating. It is important to note that the inflaton equation of state during phase $\mathrm{I}, \omega_{\phi}$, is taken to be different than that of phase II, $\omega_{\phi}$. This is where we will 
again consider lattice simulation results as another important input.

Now that we have identified the full reheating phase in terms of two distinct stages, we will numerically solve all those equations self-consistently. With the appropriate dimensionless rescaled variables for the inflaton and radiation energy densities,

$$
\Phi=\frac{\rho_{\phi} a^{3\left(1+w_{\phi}\right)}}{m_{\phi}^{\left(1-3 w_{\phi}\right)}}, \quad R(t)=\rho_{R} a^{4},
$$

the governing equations for the effective dynamical preheating phase turn into the following form:

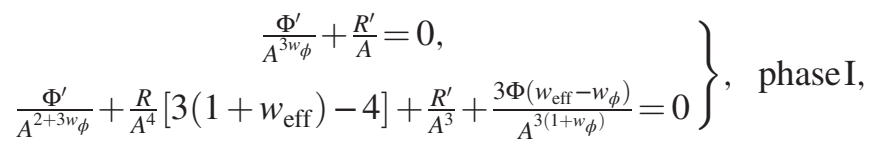

and the associated governing equations for the subsequent perturbative phase will reduce into

$$
\left.\begin{array}{c}
\Phi^{\prime}+C_{1}\left(1+w_{\phi}^{1}\right) \frac{A^{1 / 2} \Phi}{X}=0, \\
R^{\prime}-C_{1}\left(1+w_{\phi}^{1}\right) \frac{A^{\frac{3\left(1-2 w_{\phi}^{1}\right)}{2}}}{X}=0
\end{array}\right\}, \quad \text { phase II. }
$$

The rescaled scale factor is defined as $A=\frac{a}{a_{\text {end }}}$. A prime represents derivative with respect to $A$. The constant $C_{1}$ and redefined variables are

$$
X=\frac{\Phi}{A^{3 w_{\phi}^{1}}}+\frac{R}{A} ; \quad C_{1}=\frac{\sqrt{3} M_{p} \Gamma_{\phi}}{m_{\phi}^{2}} .
$$

$m_{\phi}$ is the mass of the inflaton. In next section, we will describe the methodology for solving the above set of equations numerically.

\section{PROCEDURE FOR NUMERICAL ANALYSIS AND BOUNDARY CONDITIONS}

Let us describe the strategy of our numerical study. We first identify the inflation model-dependent input parameters as $N_{k}, H_{k}$, and $V_{\text {end }}$ for a particular CMB scale $k$. For a given a canonical inflaton potential $V(\phi)$, the inflationary $e$-folding number $N_{k}$ and Hubble constant $H_{k}$ can be expressed as

$$
\begin{aligned}
& N_{k}=\log \left(\frac{a_{\mathrm{end}}}{a_{k}}\right)=\int_{\phi_{\text {end }}}^{\phi_{k}} \frac{3}{2} \frac{V(\phi)}{V^{\prime}(\phi)} d \phi=\int_{\phi_{k}}^{\phi_{\text {end }}} \frac{|d \phi|}{\sqrt{2 \epsilon_{v}} M_{p}}, \\
& H_{k}=\frac{1}{3 M_{p}^{2}} V\left(\phi_{k}\right)=\frac{\pi M_{p} \sqrt{r_{k} A_{s}}}{\sqrt{2}},
\end{aligned}
$$

where the field values at a particular scale $k,\left(\phi_{\text {end }}, \phi_{k}\right)$ are computed from the condition of the end of inflation:

$$
\epsilon\left(\phi_{\text {end }}\right)=\frac{1}{2 M_{p}^{2}}\left(\frac{V^{\prime}\left(\phi_{\mathrm{end}}\right)}{V\left(\phi_{\mathrm{end}}\right)}\right)^{2}=1
$$

and equating a particular value of scalar spectral index with $n_{s}\left(\phi_{k}\right)$. Therefore, we will get explicit relations between $\left(N_{k}, n_{s}^{k}\right)$ and $\left(H_{k}, n_{s}^{k}\right)$. The well-known inflationary input parameters can be found out from the following equations:

$$
n_{s}^{k}=1-6 \epsilon\left(\phi_{k}\right)+2 \eta\left(\phi_{k}\right), \quad r_{k}=16 \epsilon\left(\phi_{k}\right),
$$

which are expressed in terms of slow-roll parameters

$$
\epsilon_{v}=\frac{M_{p}^{2}}{2}\left(\frac{V^{\prime}}{V}\right)^{2} ; \quad\left|\eta_{v}\right|=M_{p}^{2} \frac{\left|V^{\prime \prime}\right|}{V} .
$$

The reheating parameters $N_{\text {re }}$ and $T_{\text {rad }}$ will implicitly depend upon the scalar spectral index $n_{s}^{k}$ for a given scale. The above expression can be inverted to find $\phi_{k}$ in terms of the scalar spectral index. After identifying all required parameters from inflation, we will set the initial conditions for subsequent reheating dynamics. Using all these relations among those parameters, one can establish the connection between $\mathrm{CMB}$ anisotropy and reheating through inflation.

\section{A. Phase-I initial condition}

The initial conditions for phase I of the reheating dynamics (effective nonperturbative era) are set by the end of inflation at $A=1$ and Eq. (38). Those are as follows:

$$
\begin{aligned}
& \Phi(A=1)=\frac{3}{2} \frac{V_{\mathrm{end}}(\phi)}{m_{\phi}^{4}}, \\
& R(A=1)=\frac{3\left(\omega_{\mathrm{eff}}-\omega_{\phi}\right)}{1-3 \omega_{\mathrm{eff}}} \Phi(A=1),
\end{aligned}
$$

where $V_{\text {end }}(\phi)$ is defined at the end of inflation, fixed by $\phi_{\text {end }}$. The initial Hubble expansion rate is expressed as $H_{I}=\rho_{\phi}^{\text {end }} / 3 M_{p}^{2}$.

Subsequent perturbative dynamics will now crucially depend on the end point of the first phase of reheating, namely, phase I. On this issue, we rely on the actual nonperturbative lattice simulation results $[37,41,60]$ considering a specific model of reheating where the inflaton field is assumed to couple with the reheating field. This system has been studied quite extensively $[62,63]$ in the literature by using the publicly available numerical package LATTICEEASY [64] and its parallelized version CLUSTEREASY [65]. The nonperturbative analysis for different inflationary models has been proved to yield some universal results which will be our important input for the numerical analysis. Extensive works on nonperturbative reheating analysis yields an important fact that only the $50 \%$ of the total comoving inflaton energy density is getting transferred into the daughter field. Additionally, 
the inflaton equation of state tends to achieve a steady-state value depending upon the power-law form of the inflaton potential near its minimum. For example, if one assumes the inflaton potential to be of power-law form $V \sim \phi^{n}$, for a chaotic-type model, namely, $n=2$, the nonperturbative phase ends with a steady value of the equation of state $\sim 0.2$. However, for other value of $n \geq 4$, the equation of state approaches $\omega=\frac{1}{3}$ at the end point of the nonperturbative reheating. These are the crucial quantitative results from nonperturbative preheating dynamics we will be utilizing in our analysis for the phase-II dynamics.

\section{B. Phase-II initial condition}

After the phase-I dynamics, the perturbative dynamics will automatically follow. However, an important point would be to identify the appropriate boundary conditions. The starting moment of phase II will be set by the normalized scale factor $A_{\text {npre }}=a_{\text {npre }} / a_{\text {end }}$, which is the ratio between the scale factor at the end of the effective nonperturbative epoch, namely, phase I $a_{\text {npre }}$, and the end of the inflation. The initial conditions for the dimensionless comoving densities are

$$
\Phi=\Phi\left(A_{\text {npre }}\right) ; \quad \frac{R\left(A_{\text {npre }}\right)}{R\left(A_{\text {npre }}\right)+\Phi\left(A_{\text {npre }}\right)} \simeq \frac{1}{2} .
$$

It is important to realize that the initial condition is determined by $50 \%$ decay of the total comoving energy density $\rho_{T}$. Furthermore, we have numerically checked that our results do not seem to depend both qualitatively as well as quantitatively much on the amount of decay within $40 \%-60 \%$ of the total energy at the end of phase I. For the analysis, we further assume the inflaton equation of state $\omega_{\phi}^{1} \simeq 0.2$ irrespective of the models under consideration. This approximate value is again another important input from the lattice simulation. For comparison, we also consider the cases where either phase-I or phase-II evolution completely governs the reheating dynamics.

\section{Determining the reheating parameters}

Once we numerically solve the reheating dynamics, we define one of the important parameters called reheating temperature $T_{\text {re }}$, which is generically identified as the radiation temperature $T_{\text {rad }}$ when the condition $H(t)=\Gamma_{\phi}$ is satisfied:

$H\left(A_{\mathrm{re}}\right)^{2}=\left(\frac{\dot{A}_{\mathrm{re}}}{A_{\mathrm{re}}}\right)^{2}=\frac{\rho_{\phi}\left(\Gamma_{\phi}, A_{\mathrm{re}}, n_{s}^{k}\right)+\rho_{R}\left(\Gamma_{\phi}, A_{\mathrm{re}}, n_{s}^{k}\right)}{3 M_{p}^{2}}=\Gamma_{\phi}^{2}$,

where $A_{\text {re }}$ is the normalized scale factor at the end of the reheating. Accordingly, the reheating temperature in terms of the radiation temperature $\left(T_{\mathrm{rad}}\right)$ is expressed as

$$
T_{\mathrm{re}}=T_{\mathrm{rad}}^{\mathrm{end}}=\left(\frac{30}{\pi^{2} g_{*}(T)}\right)^{1 / 4} \rho_{R}\left(\Gamma_{\phi}, A_{\mathrm{re}}, n_{s}^{k}\right)^{1 / 4} .
$$

Furthermore, the $e$-folding number during reheating $N_{\text {re }}$ consists of two contributions born out of two distinct phases as

$$
\begin{gathered}
N_{\text {re }}=\log \left(\frac{a_{\text {re }}}{a_{\text {end }}}\right)=\log \left(\frac{a_{\text {re }}}{a_{\text {npre }}} \frac{a_{\text {npre }}}{a_{\text {end }}}\right)=N_{\text {pre }}+N_{\text {npre }}, \\
N_{\text {pre }}=\log \left(\frac{a_{\text {re }}}{a_{\text {npre }}}\right), \quad N_{\text {npre }}=\log \left(\frac{a_{\text {npre }}}{a_{\text {end }}}\right),
\end{gathered}
$$

where $N_{\text {pre }}$ and $N_{\text {npre }}$ are the $e$-folding number during the perturbative and effective nonperturbative region, respectively. Combining Eqs. (8) and (53), we obtain the most important modification of Eq. (8) relating the reheating and inflationary parameters:

$$
T_{\text {re }}=\left(\frac{43}{11 g_{\mathrm{re}}}\right)^{1 / 3}\left(\frac{a_{0} T_{0}}{k}\right) H_{k} e^{-N_{k}} e^{-N_{\text {npre }}} e^{-N_{\text {pre }}} .
$$

Now connecting Eqs. (51), (52), and (55), we can establish one-to-one correspondence between $T_{\text {re }}$ and $\Gamma_{\phi}$.

As described before, we will consider three possible cases and compare the results:

$$
\begin{array}{llll}
\text { case I } & N_{\text {npre }} \neq 0, & N_{\text {pre }} \neq 0 & \text { phase I }+ \text { phase II }, \\
\text { case II } & N_{\text {npre }} \neq 0, & N_{\text {pre }}=0 & \text { Kamionkowski } \text { et al. } .6], \\
\text { case III } & N_{\text {npre }}=0, & N_{\text {pre }} \neq 0 & \text { phase II, discussed in the previous section. }
\end{array}
$$

To this end, let us specifically mention case II, when perturbative dynamics ceases to exist. This particular procedure, proposed in Ref. [11], has been studied quite extensively in the literature [38]. In this particular phase, dynamics is solely governed by the effective equation of state $\omega_{\text {eff }}$. The explicit decay of inflaton does not appear in the computation. However, information about the decay constant $\Gamma_{\phi}$ is extracted from the equilibrium condition 
$\Gamma_{\phi}=H$, where the reheating temperature $\left(T_{\mathrm{re}}\right)$ is defined as $T_{\mathrm{re}}=0.2\left(\frac{200}{g_{*}}\right)^{1 / 4}\left(\Gamma_{\phi} M_{p l}\right)^{1 / 2}$, with $g_{*}$ being the effective number of relativistic degrees of freedom. However, not to ignore an important difference between the phase I described before and the approach devised in Ref. [11] or case II for the present study is the additional conservation equation (38). This essentially differentiates the regime of applicability of these two approaches. Phase-I dynamics is assumed to be applicable in the early nonperturbative regime. Whereas, since condition (38) does not exist, the original Kamionkowski et al. [11] approach is effectively applicable throughout the full period of reheating without any microscopic details. Furthermore, the value of $\omega_{\text {eff }}$ is no longer constrained to be very close to the inflaton equation of state during phase I. This relation essentially helps us to compare the results for various scenarios we consider. To avoid symbol confusion whenever we study case II, we use the symbol $\omega_{\text {eff }}^{K}$ instead of $\omega_{\text {eff }}$, which we reserve for twophase reheating dynamics.

\section{MAXIMUM RADIATION TEMPERATURE AND REHEATING TEMPERATURE: ANALYTIC STUDY}

Before moving on to a particular model, let us analytically estimate the maximum reheating temperature and its dependence upon the initial condition following the same line as before. Considering the standard definition of the radiation temperature $T_{\text {rad }}=\left(\frac{30}{\pi^{2} g_{*}} \rho_{R}\right)^{1 / 4}$ and computing the radiation energy density during phase II following Eqs. (36), (37), (39), and (40), the approximate radiation temperature assumes the following form (see Appendix A for details of the calculation):

$$
T_{\mathrm{rad}}=\left(\frac{\rho_{\phi}^{\mathrm{in}} \Gamma_{\phi}\left(1+\omega_{\phi}^{1}\right)}{\beta x^{4} H_{\mathrm{in}}}\left[\frac{2}{5-c}\left(x^{(5-c) / 2}-1\right)+\frac{\rho_{R}^{\mathrm{in}}}{\rho_{\phi}^{\mathrm{in}}}\left(\frac{1-x^{(c+3) / 2}}{c+3}+\frac{H_{\mathrm{in}}}{\Gamma_{\phi}\left(1+\omega_{\phi}^{1}\right)}\right)\right]\right)^{1 / 4},
$$

where $x, \beta, c$, and $H_{\text {in }}$ are expressed as

$$
x=\frac{a}{a_{\text {npre }}}, \quad \beta=\frac{\pi^{2} g_{*}(T)}{30}, \quad c=3 \omega_{\phi}^{1}, \quad H_{\text {in }}=\frac{\sqrt{\rho_{\phi}^{\text {in }}}}{\sqrt{3} M_{P}} .
$$

In the above expression, $\rho_{\phi}^{\text {in }}$ and $\rho_{R}^{\text {in }}$ represent inflaton and radiation energy density, respectively, at the end of phase I or the beginning of phase II:

$$
\rho_{\phi}^{\text {in }}=\rho_{\phi}\left(a=a_{\text {npre }}\right), \quad \rho_{R}^{\text {in }}=\rho_{R}\left(a=a_{\text {npre }}\right) .
$$

The maximum radiation temperature is defined at the point $x_{\max }=a_{\max } / a_{\text {npre }}$, where $\frac{d T_{\text {rad }}}{d x}=0$, which gives us the maximum radiation temperature for two-phase reheating expressed in terms of dimensionless comoving densities:

$$
\begin{gathered}
T_{\mathrm{rad}}^{\max } \simeq D^{1 / 4}\left[1+\frac{(3+c) R\left(A_{\mathrm{npre}}\right)}{8 \Phi\left(A_{\mathrm{npre}}\right) A_{\mathrm{npre}}^{1-c}}\left(\frac{1-x_{\mathrm{max}, p}^{(c+3) / 2}}{c+3}+\frac{\sqrt{\Phi\left(A_{\mathrm{npre}}\right) A_{\mathrm{npre}}^{-3\left(1+\omega_{\phi}^{1}\right)} m_{\phi}^{4}}}{\sqrt{3} M_{p} \Gamma_{\phi}\left(1+\omega_{\phi}^{1}\right)}\right)\right] \\
D=\left(\frac{2 \Gamma_{\phi} \sqrt{3 M_{p}^{2} \Phi\left(A_{\mathrm{npre}}\right) A_{\mathrm{npre}}^{-3\left(1+\omega_{\phi}^{1}\right)} m_{\phi}^{4}}}{(3+c) \beta x_{\max , p}^{4}}\right)^{1 / 4}, \quad x_{\max , p}=\left(\frac{8}{3+c}\right)^{2 /(5-c)}
\end{gathered}
$$

One particularly notices the correction term in the maximum radiation temperature due to initial comoving radiation density $R\left(A_{\text {npre }}\right)$ at the beginning of phase II. It boils down to well-known expression $T_{\mathrm{re}}^{\max }=D^{1 / 4}$ in the $R\left(A_{\text {npre }}\right)=0$ limit the same as Eq. [46]. In the above expression, we ignored the contribution of dark matter. However, generically during the reheating period, dark matter is not the dominant component; therefore, the numerical value of the reheating temperature will not be affected. The analytic expression for the dimensionless comoving density during phase II related to the density at the end of inflation will be

$$
\Phi\left(A_{\text {npre }}\right)=\left(1-3 \omega_{\text {eff }}\right) \Phi(A=1) A_{\text {npre }}^{-3 \omega_{\text {eff }}}
$$

where $A_{\text {npre }}$ is the normalized scale factor at the end of the effective dynamics (phase I): 


$$
A_{\text {npre }}=\frac{1-3 \omega_{\mathrm{eff}}}{3 \omega_{\mathrm{eff}}} .
$$

One of our important results from the above expression for the maximum reheating temperature is the highest radiation temperature, which is defined at $T_{\mathrm{rad}}^{\max }=T_{\mathrm{re}}^{\max }$ corresponding to a given $n_{s}^{\max }$. As we change the value of $\omega_{\text {eff }}=\left(10^{-3} \rightarrow 10^{-6}\right)$, the maximum reheating temperature changes as $T_{\mathrm{re}}^{\max }=\left(10^{13} \rightarrow 10^{10}\right) \mathrm{GeV}$. Once we set $R\left(A_{\text {npre }}\right)=0$, the maximum reheating temperature becomes $T_{\mathrm{re}}^{\max } \sim 10^{15} \mathrm{GeV}$ as expected [see Eqs. [46] and (18)]. Proceeding further, we can also obtain the approximation expression for the reheating temperature itself. Utilizing the expression of the Hubble constant at the equilibrium point $\left(H_{\mathrm{re}}=\Gamma_{\phi}\right)$ and subsequent entropy conservation, one arrives at the following expression:

$T_{\mathrm{re}}^{4} \simeq \frac{x_{\mathrm{re}}^{-4} \rho_{\phi}^{\text {in }}}{\beta}\left[\frac{G^{4} \beta}{\rho_{\phi}^{\text {in }}}+\frac{5-c}{2(c+3)} \frac{\rho_{R}^{\text {in }}}{\rho_{\phi}^{\text {in }}}\left(\frac{G^{4} \beta}{\rho_{\phi}^{\text {in }}}-\frac{\rho_{R}^{\text {in }}}{\rho_{\phi}^{\text {in }}}\right) x_{\mathrm{re}}^{c-1}\right]$,

where $x_{\mathrm{re}}=a_{\mathrm{re}} / a_{\mathrm{npre}}$ can be recognized as

$$
x_{\mathrm{re}}=\left(\frac{\alpha}{\eta}\right)^{1 /(c-1)} .
$$

Here

$$
\begin{aligned}
\alpha= & \frac{G^{4} \beta}{\rho_{\phi}^{\text {in }}}, \\
\eta= & \frac{5-c}{2}\left(\frac{G^{4} \beta}{\rho_{\phi}^{\text {in }}}-\frac{\rho_{R}^{\text {in }}}{\rho_{\phi}^{\text {in }}}\right)\left[\frac{\rho_{R}^{\text {in }}}{(c+3) \rho_{\phi}^{\text {in }}}+\frac{5-c}{2} \frac{3 M_{p}^{2} H_{\text {in }}^{2}}{\rho_{\phi}^{\text {in }}\left(1+\omega_{\phi}^{1}\right)^{2}}\right. \\
& \left.\times\left(\frac{G^{4} \beta}{\rho_{\phi}^{\text {in }}}-\frac{\rho_{R}^{\text {in }}}{\rho_{\phi}^{\text {in }}}\right)\right] .
\end{aligned}
$$

The detailed derivation of all the aforementioned equations for the reheating temperature is in Appendix B. Now we will consider a class of inflationary models of inflation and analyze our proposal of two-phase reheating scenario.

\section{INFLATION MODELS AND NUMERICAL RESULTS}

Based on our methodology discussed above, we will now consider a class of inflationary models for which the inflaton potentials assume quadratic form. We will also point out the regime of validity of the effective nonperturbative and perturbative era for the different inflationary models. After the inflation, the inflaton field generically oscillates around the minimum of its potential $V(\phi)$. Reheating fields coupled with the oscillating inflaton is generically prone to nonperturbative particle production. Our objective is to replace this nonperturbative dynamics by an effective dynamical equation, which is solely governed by the effective equation of state, $\omega_{\text {eff }}$ supplemented with the additional constraint relation Eq. (38). We have already observed that, during phase I, $\omega_{\text {eff }}$ is close to that of the inflaton equation of state, $\omega_{\phi}$. Near the minimum of the potential, if the form is taken to be power law as $\propto \phi^{n}$, over multiple oscillations, the average inflaton equation of state is expressed as [66]

$$
\omega_{\phi}=\frac{P_{\phi}}{\rho_{\phi}} \approx \frac{\left\langle\phi V^{\prime}(\phi)-2 V(\phi)\right\rangle}{\left\langle\phi V^{\prime}(\phi)+2 V(\phi)\right\rangle}=\frac{n-2}{n+2} .
$$

For the $n=2$ model, $\omega_{\phi}$ assumes a dustlike equation of state $\left(\omega_{\phi}=0\right)$. Throughout the subsequent study, we consider those inflationary models which have quadratic potential near their minimum. Therefore, during phase I of reheating, we set $\omega_{\phi}=0$. To this end, let us emphasize again that during phase II, when the reheating dynamics enter into the perturbative phase, we assume the inflaton equation of state $\omega_{\phi}^{1} \simeq 0.2$, which is one of the important lattice simulation results mentioned earlier. Furthermore, we analyze phase-I dynamics considering two specific choices of the effective equation of state $\omega_{\text {eff }}=\left(\omega_{\phi}+10^{-3}, \omega_{\phi}+10^{-6}\right)$ which are close to $\omega_{\phi}$.

\section{A. Plots and important model-independent observations}

Before we go into a detailed discussion on various inflationary models, let us first illustrate different plots and important model-independent observations. For each model, we have drawn two different plots: one in $\left(\Gamma_{\phi}\right.$ vs $\left.N\right)$ space where it shows the variation of reheating $e$-folding number $N$ depending upon the inflaton decay constant. As noted earlier, we have considered different scenarios. For our proposed two-phase reheating scenario (case I), we have studied two possible values of phase-I effective equation of state, $\omega_{\text {eff }}=10^{-3}$, corresponding to the solid green and solid black curves, and $\omega_{\text {eff }}=10^{-6}$, corresponding to the dotted green and dotted black curves. For all cases, $\omega_{\phi}=0$. One of the most important outcomes of our analysis is the emergence of a critical inflaton decay constant $\Gamma_{\phi}=\Gamma_{\phi}^{\text {cri }}$ denoted by red dots associated with each particular $\omega_{\text {eff. }}$ This indicates the fact that, for $\Gamma_{\phi}>\Gamma_{\phi}^{\mathrm{cri}}$, the reheating period will be dominated by phase-I, effective nonperturbative dynamics; otherwise, it is perturbative dominated. The critical value of the inflaton decay constant increases with the decreasing $\omega_{\text {eff }}$. This can be understood from several interconnecting physical effects. First of all, most importantly, Eq. (38):

$$
R(A=1)=\frac{\left(\omega_{\mathrm{eff}}-\omega_{\phi}\right)}{1-3 \omega_{\mathrm{eff}}} \Phi(A=1),
$$

which not only fixes the approximate value of $\omega_{\text {eff }}$ but also sets the initial condition for phase-I dynamics. Furthermore, the larger the value of $\omega_{\text {eff }}$, the higher will 
be initial radiation density $R(A=1)$, which automatically leads to a smaller value of phase-I $e$-folding number $N_{\text {npre }}$. Therefore, a particular $\Gamma_{\phi}$ will naturally lead to larger $N_{\text {pre }}$, as associated with each $\Gamma_{\phi}$ there exists a reheating temperature which follows from $T_{\mathrm{re}} \propto e^{-N_{\mathrm{re}}}=e^{-\left(N_{\mathrm{pre}}+N_{\text {npre }}\right)}$. On the other hand, critical $\Gamma_{\phi}^{\text {cri }}$ is a point where $N_{\text {pre }}=N_{\text {npre }}$ in $N$ vs $\Gamma_{\phi}$ space. From these two conditions, one can argue that a transition from perturbative to nonperturbative reheating phase would occur for a larger critical value $\Gamma_{\phi}^{\text {cri }}$ for a larger $\omega_{\text {eff }}$ value. Given a reheating model with specific inflaton-daughter field interaction, we have also discussed the existence of critical inflation decay constant $\Gamma_{\phi}^{\mathrm{cri}}($ model$)$, which were shown by vertical red lines in the plots. From the theoretical values of the critical inflaton decay constant, $\left(\Gamma_{\phi}\right.$ vs $\left.N_{k}\right)$ plots indicate that the value of $\omega_{\text {eff }}$ must lie within $\left(10^{-3}, 10^{-6}\right)$ irrespective of the inflationary models considered. At this point, let us understand the physical meaning of nonvanishing initial radiation density $\rho_{R}(A=1) \simeq\left(10^{-3}, 10^{-6}\right) \rho_{\phi}^{\text {in }}$ considering $\omega_{\text {eff }}=$ $\left(10^{-3}, 10^{-6}\right)$. We replace the full nonperturbative dynamics by an effective dynamics, which naturally does not capture the complete picture. Typically, the nonperturbative phase contains three distinct phases: the parametric resonance phase, thermalization phase, and steady-state phase. And this is the initial parametric resonance phase, where explosive particle production can naturally give rise to required initial radiation density $\rho_{R}(A=1) \simeq 10^{-6}-10^{-3}$ in units of total density $\rho_{\phi}$ almost instantly.

To see whether our proposed phase-I dynamics is justified or not, we compare our result with actual nonperturbative results. In order to do that, we use nonperturbative lattice simulation during the preheating, considering a specific inflaton-reheating field interaction $\frac{1}{2} g^{2} \phi^{2} \chi^{2}$. In all the lattice simulation results, the initial radiation density typically assumes $\rho_{R}(A=1) \simeq 10^{-4}$ in units of initial inflaton energy density, which essentially lies within what we have considered. Once the preheating phase reaches the steady-state condition, we again solve perturbative dynamics and found that the reheating ends at around the same value of $A_{\text {re }}$ (shown by the dashed black line) where our two-phase reheating ends for $\omega_{\text {eff }}=10^{-3}$ (solid pink line) and for $\omega_{\text {eff }}=10^{-6}$ (dashed pink line) accordingly. Therefore, our effective two-phase reheating approach seems to capture the essential properties of nonperturbative lattice results, except the nonperturbative $e$-folding number, which will be taken up in the future.

Nevertheless, for comparison, in the same plot, we also have drawn the total reheating $e$-folding number for the other two cases: dotted pink lines for case II and solid blue lines for case III mentioned before. It turns out that the total number of reheating $e$-folding number for case II, case III, and case I, $N_{\text {re }}=\left(N_{\text {npre }}+n_{\text {pre }}\right)$, is almost the same for all different values of the equation of state.
In another class of plots in ( $n_{s}$ vs $\left.T_{\text {re }}\right)$ space, we describe the variation of reheating temperature $T_{\text {re }}$ with respect to the scalar spectral index $n_{s}$. From these plots, we can read that the two-phase reheating process (case I) is crucially dependent upon the value of $\omega_{\text {eff }}$. Furthermore, case-I results are qualitatively similar to that of case II $\omega_{\text {eff }}^{K}=$ 0.212 (equation of state at the starting point of phase II in two-phase analysis). On the other hand, perturbative reheating (case-III) results are qualitatively similar to that of case II for $\omega_{\mathrm{eff}}^{K}=0$. For the usual perturbative reheating scenario (case III), the semianalytic approach discussed before reveals the existence of maximum possible reheating temperature $\sim 10^{15} \mathrm{GeV}$. Our numerical computation also indicates the same through solid blue lines. Furthermore, the case-II scenario also has the same prediction of modelindependent maximum reheating temperature $T_{\mathrm{re}}^{\max }$ irrespective of the value of its effective equation of state $\omega_{\mathrm{eff}}^{K}=$ $(0,0.212)$ shown through solid pink lines and dotted pink lines, respectively. For conventional reheating dynamics (case II and case III), the maximum reheating temperature directly corresponds to instantaneous reheating with total $e$-folding number $N_{\text {re }} \rightarrow 0$. This can also be straightforwardly connected with the maximum possible scalar spectral index $n_{s}^{\max }$. The proposed two-stage reheating dynamics (case I) instead predicts very different results in this regard. First of all, instantaneous reheating ceases to exist in this scenario because of its underlying assumptions. As $N_{\text {pre }} \rightarrow 0, N_{\text {re }} \rightarrow N_{\text {npre }}$, which automatically leads to different values of $\left(T_{\mathrm{re}}^{\max }, n_{s}^{\max }\right)$ followed from the condition $N_{\text {re }}=N_{\text {npre }}$, which naturally assumes model-independent values such as $N_{\text {npre }} \sim 6$ for $\omega_{\text {eff }}=10^{-3}$ and $N_{\text {npre }} \sim 12$ for $\omega_{\text {eff }}=10^{-6}$. The smaller the effective equation of state during phase I, the larger will be its duration $N_{\text {npre }}$, and, consequently, $T_{\mathrm{re}}^{\max }$ will be reduced. As expected from our earlier analytical calculation, the important results are the values of maximum reheating temperature $T_{\mathrm{re}}^{\max } \sim$ $\left(10^{13}, 10^{10}\right) \mathrm{GeV}$ for $\omega_{\text {eff }}=\left(10^{-3}, 10^{-6}\right)$, respectively. Physical origin of these two different limiting temperatures is clear from the fact that an increase of $T_{\text {re }}$ is directly connected with the increase of $\Gamma_{\phi}$. Hence, with the increasing temperature, reheating dynamics undergoes a transition from perturbative to nonperturbative regime at particular critical temperature $T_{\mathrm{re}}^{\text {cri }}$ associated with $\Gamma_{\phi}^{\text {cri }}$, leading to a distinct value of $N_{\text {npre }}$ which is different for different $\omega_{\text {eff }}$ values. This leads to different $T_{\mathrm{re}}^{\max }$. Therefore, an important conclusion we can arrive at is that, given the approximate estimates of model-specific critical decay width $\Gamma_{\phi}^{\mathrm{cri}}($ model $)$, the maximum reheating temperature $T_{\mathrm{re}}^{\max }$ should be within $\left(10^{10}-10^{13}\right) \mathrm{GeV}$, irrespective of the dynamics of the second phase-II and inflationary model under consideration. However, we must note that the associated maximum values of $n_{s}^{\max }$ are model dependent, which will be discussed for each model. 


\section{B. Chaotic inflation [67]}

Even though chaotic inflation is observationally disfavored, we consider this potential for its simple nature. For usual chaotic inflation, the potential looks like

$$
V(\phi)=\frac{1}{2} m^{4-n} \phi^{n},
$$

where $n=2,4,6, \ldots$. If we consider only the absolute value of the field, $n=3,5, \ldots$ can also be included. $m$ is a parameter of mass dimension. For the purpose of our study, we consider only $n=2$ mainly because $\omega_{\phi}=0$.

\section{Initial conditions for phase I}

The initial densities to solve dynamical equation during phase I can be calculated as

$$
\begin{aligned}
& \Phi(A=1)=\frac{3}{4} \frac{m^{4-n}}{m_{\phi}^{4}}\left(\frac{n M_{p}}{\sqrt{2}}\right)^{n}, \\
& R(A=1)=\frac{3\left(\omega_{\mathrm{eff}}-\omega_{\phi}\right)}{1-3 \omega_{\phi}} \Phi(A=1),
\end{aligned}
$$

where

$$
m=M_{p}\left(3 \pi^{2} r_{k} A_{\delta \phi}\right)^{1 /(4-n)}\left(\frac{1-n_{s}^{k}}{n(n+2)}\right)^{n /[2(4-n)]} .
$$

$A_{\delta \phi} \sim 10^{-9}$ is the amplitude of the inflaton fluctuation which is measured from CMB observation. $m_{\phi}$ is defined as the second derivative of the inflaton potential. To establish the connection among inflationary and reheating parameters, the inflationary $e$-folding number $N_{k}$ and tensor-toscalar ratio $r_{k}$ are similarly calculated as

$$
N_{k}=\frac{n+2}{2\left(1-n_{s}^{k}\right)}-\frac{n}{4}, \quad r_{k}=\frac{8 n}{n+2}\left(1-n_{s}^{k}\right) .
$$

\section{Initial conditions for phase II}

Additionally, the initial conditions for phase II will be set at the normalized scale factor $A_{\text {npre }}$ where phase I ends. The conditions are

$$
\Phi=\Phi\left(A_{\text {npre }}\right), \quad \frac{R\left(A_{\text {npre }}\right)}{R\left(A_{\text {npre }}\right)+\Phi\left(A_{\text {npre }}\right)} \simeq \frac{1}{2}
$$

To establish the relation between the reheating temperature $\left(T_{\text {re }}\right)$ and inflationary index $\left(n_{s}^{k}\right)$, we follow the methodology explained in the previous section.

\section{Observations}

Important results for chaotic inflation are depicted in Fig. 4. As stated at length, the initial effective equation of state $\omega_{\text {eff }}$ plays a crucial role in driving the whole reheating dynamics. For our purpose, we took two sample values $\left(10^{-3}, 10^{-6}\right)$. According to these two values, the critical values of the inflaton decay constants are found to be $\Gamma_{\phi}^{\mathrm{cri}}=\left(2.46 \times 10^{3}, 2.73 \times 10^{-7}\right) \mathrm{GeV}$. Similarly, we can address critical values (transition from perturbative to nonperturbative reheating) in terms of the reheating temperature. For this model, the critical values of the reheating temperature are set to be $T_{\mathrm{re}}^{\mathrm{cri}} \simeq\left(2.7 \times 10^{10}, 3 \times 10^{5}\right) \mathrm{GeV}$ for the equation of state $\omega_{\text {eff }}=\left(10^{-3}, 10^{-6}\right)$. This entails the fact that if $\Gamma_{\phi}>\Gamma_{\phi}^{\mathrm{cri}}\left(T_{\mathrm{re}}>T_{\mathrm{re}}^{\mathrm{cri}}\right)$, the reheating phase will be dominated by the nonperturbative process.

For concreteness, let us bring specific reheating models into consideration. We have discussed three different interaction models with associated nonperturbative constraints equations (30), (26), and (34). Associated with those, we have theoretical values of the critical inflaton decay constants $\Gamma_{\phi}^{\text {cri }}($ model $)=(0.003,0.5,11.8) \mathrm{GeV}$, respectively. The first two values correspond to inflaton decaying into the scalar particle, and the third one corresponds to decaying into a pair of fermionic particles. Interestingly, comparing those numerical and theoretical values of $\Gamma_{\phi}^{\text {cri }}$, one can observe that the initial effective equation of state $\omega_{\text {eff }}$ during phase I must lie within $\left(10^{-3}, 10^{-6}\right)$. This essentially suggests that all three models of inflaton interaction will lead to initial radiation density within the value $\left(10^{-3}, 10^{-6}\right)$ instantaneously, which we can immediately read off from Fig. 3 .

In all the reheating scenarios discussed and proposed so far, there exists a model-independent maximum reheating temperature. However, the associated maximum value of the spectral index $n_{s}^{\max }$ turned out to be model dependent. In the conventional perturbative reheating discussed before, and also the constraints from the reheating (case-II) scenario, $N_{\text {re }} \rightarrow 0$ provides the condition for $n_{s}^{\max }$. For the two-phase reheating scenario (case I), the phase-I effective dynamics is inevitable, which leads to different condition $N_{\text {re }} \approx N_{\text {npre }}$ for the maximum possible $n_{s}^{\max }$ compatible with CMB observation. Furthermore, for each model, one can define minimum spectral index $n_{s}^{\min }$ which can be associated with the minimum possible reheating temperature set by BBN constraints [68-71], which is $T_{\mathrm{re}}^{\min }=10^{-2} \mathrm{GeV}$. Taking into account both the possibilities, for case I, we obtain the possible bound on the spectral index $0.9628 \leq n_{s} \leq 0.9653$ and $0.9628 \leq n_{s} \leq 0.9649$ for $\omega_{\text {eff }}=\left(10^{-3}, 10^{-6}\right)$, respectively. For case II [11], the bound is $0.955 \leq n_{s} \leq 0.9654$ and $0.9629 \leq n_{s} \leq 0.9654$ for $\omega_{\mathrm{eff}}^{K}=(0,0.212)$, respectively. Additionally, for purely perturbative dynamics case III, one obtains $0.9555 \leq n_{s} \leq$ 0.9657. Important to recall at this point, all these bounds are consistent with $\mathrm{CMB}$ within $2 \sigma$ error of $n_{s}$. From the maximum $n_{s}^{\max }$, the maximum value of the inflationary $e$-folding number $\left(N_{k}^{\max }\right)$ can be obtained. For example, for the case-I scenario we have $N_{k}^{\max } \simeq(57,56)$ with effective 


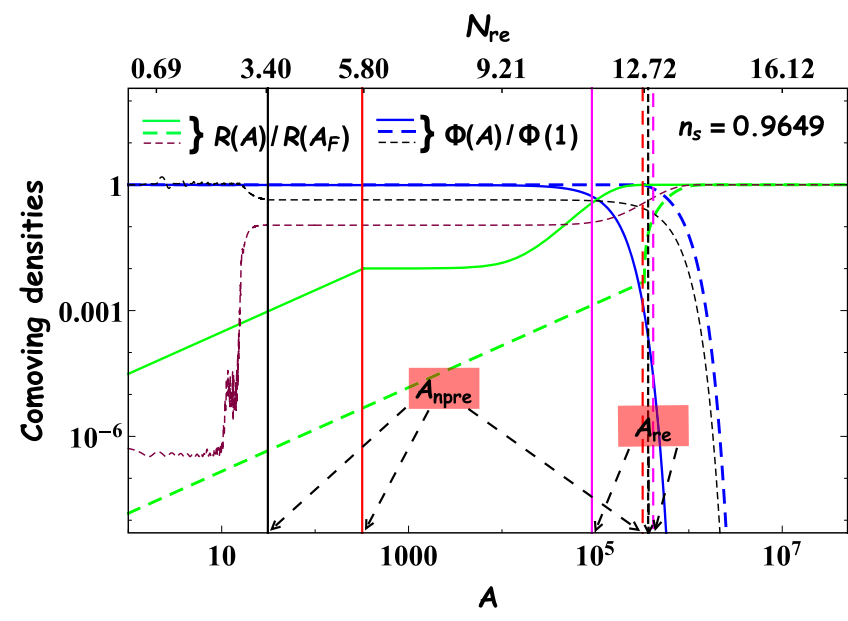

FIG. 3. We plot the evolution of the different energy components (inflaton and radiation) with the normalized scale factor for chaotic inflation model with $n=2$. The blue and green curves indicate the variation of comoving densities, the inflaton, and radiation density, respectively, for our proposed two-phase dynamics (case I). The red and pink lines represent the normalized scale factor at the ending of phase I and II accordingly. Furthermore, the solid and dashed curves correspond to the two different values of the effective equation of state $\omega_{\text {eff }}=$ $\left(10^{-3}, 10^{-6}\right)$, whereas the result for considering the standard nonperturbative lattice simulation, during phase I, is shown by the dashed black and brown lines.

equation of state $\omega_{\text {eff }}=\left(10^{-3}, 10^{-6}\right)$, respectively, whereas, for case II, $N_{k}^{\max } \simeq 57$ and, for case III, $N_{k}^{\max } \simeq 58$.

The variation of the reheating temperature as a function of the spectral index for the different reheating mechanism is shown in Fig. 4. The behavior of the reheating temperature with respect to $n_{s}$ appears to be model independent.

\section{Axion inflation $[72,73]$}

The potential for the axion or natural inflation is

$$
V(\phi)=\Lambda^{4}\left[1-\cos \left(\frac{\phi}{f}\right)\right]
$$

where $(\Lambda, f)$ are the scale of inflation and axion decay constant of this present model. By tuning the value of the decay constant, this model is marginally consistent with the recent observation [74]. To be consistent with CMB data, we consider two sample super-Planckian values of the axion decay constant, $f=(10,50) M_{p}$. The scale of this inflation, $\Lambda$, is fixed by the CMB normalization.

\section{Initial conditions for phase I}

The initial conditions to solve the differential equations for the effective nonperturbative era are set at the end of inflation to be

$$
\begin{aligned}
& \Phi(A=1)=\frac{3}{2} \frac{2 \Lambda^{4} M_{p}^{2}}{\left(2 f^{2}+M_{p}^{2}\right) m_{\phi}^{4}}, \\
& R(A=1)=\frac{3\left(\omega_{\mathrm{eff}}-\omega_{\phi}\right)}{1-3 \omega_{\mathrm{eff}}} \Phi(A=1),
\end{aligned}
$$

where

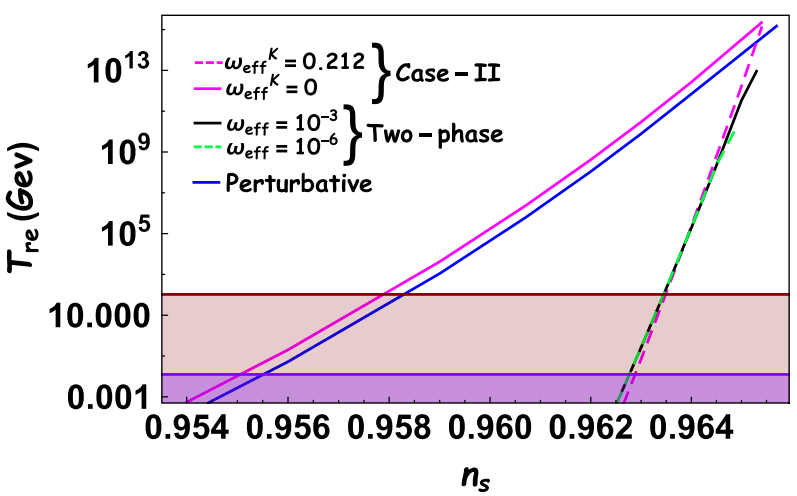

FIG. 4. We plot, on the left side, the variation of the $e$-folding number as a function of the inflation decay width $\left(\Gamma_{\phi}\right)$ and, on the right side, variation of the reheating temperature $\left(T_{\text {re }}\right)$ as a function of $n_{s}$ for the chaotic inflation model with $n=2$. The plot on the left side, variations of $N_{\text {npre }}$ ( $e$-folding number during first phase of reheating) and $N_{\text {pre }}(e$-folding number during perturbative reheating) are shown by black and green lines (solid and dashed), respectively, for two different values of $\omega_{\text {eff }}=\left(10^{-3}, 10^{-6}\right)$. The intersection points of $N_{\text {npre }}$ and $N_{\text {pre }}$ for different values of $\omega_{\text {eff }}$ are shown by a red circle. The blue and dashed pink lines indicate the variation of the $e$-folding number during reheating for purely perturbative and the analysis given by Kamionkowski et al. [11] with $\omega_{\text {eff }}^{K}=0$, respectively. The thick dashed, thin dashed, and solid red lines correspond to the three different values of the decay constant at the transition point of nonperturbative to the perturbative era from the theoretical point of view provided by Eqs. (34), (26), and (30), respectively. All plots are drawn within $2 \sigma$ range of $n_{s}$ [3]. The light brown region is below the electroweak scale $T_{\text {ew }} \sim 100 \mathrm{GeV}$, and the violet region below $10^{-2} \mathrm{GeV}$ would ruin the predictions of $\mathrm{BBN}$. 
$\Lambda=\left(\frac{3 \pi^{2} M_{p}^{2} A_{s}\left(f^{4}\left(1-n_{s}\right)^{2}-M_{p}^{4}\right)}{2 f^{2}}\right)^{1 / 4}, \quad m_{\phi}=\frac{\Lambda^{2}}{f}$.

In addition, the inflationary $e$-folding number $N_{k}$ and tensor-to-scalar ratio $r_{k}$ for the natural inflation model are expressed in terms of scalar spectral index and model parameters as

$$
\begin{aligned}
N_{k} & =\frac{f^{2}}{M_{p}^{2}} \ln \left(\frac{2 f^{2}\left(f^{2}\left(1-n_{s}\right)+M_{p}^{2}\right)}{\left(2 f^{2}+M_{p}^{2}\right)\left(f^{2}\left(1-n_{s}\right)-M_{p}^{2}\right)}\right), \\
r_{k} & =4\left(\frac{f^{2}\left(1-n_{s}\right)-M_{p}^{2}}{f^{2}}\right) .
\end{aligned}
$$

The initial condition for the phase-II dynamics will be the same as chaotic inflation has given in Eq. (73).

\section{Observations}

The main results of the axion inflationary model are depicted in Fig. 5. As has been mentioned earlier, we have considered two sample values of the axion decay constant $f=(10,50) M_{p}$. For a fixed value of axion decay constant $f=10 M_{p}$, the critical values of the inflaton decay constant assume $\Gamma_{\phi}^{\mathrm{cri}} \simeq\left(3.7 \times 10^{4}, 1.1 \times 10^{-7}\right) \mathrm{GeV}$, and those of the reheating temperatures are $T_{\mathrm{re}}^{\mathrm{cri}} \simeq\left(4 \times 10^{10}, 1.8 \times 10^{5}\right) \mathrm{GeV}$. Similarly, for $f=50 M_{p}, \Gamma_{\phi}^{\text {cri }} \simeq\left(2.7 \times 10^{4}, 1.1 \times 10^{-7}\right) \mathrm{GeV}$
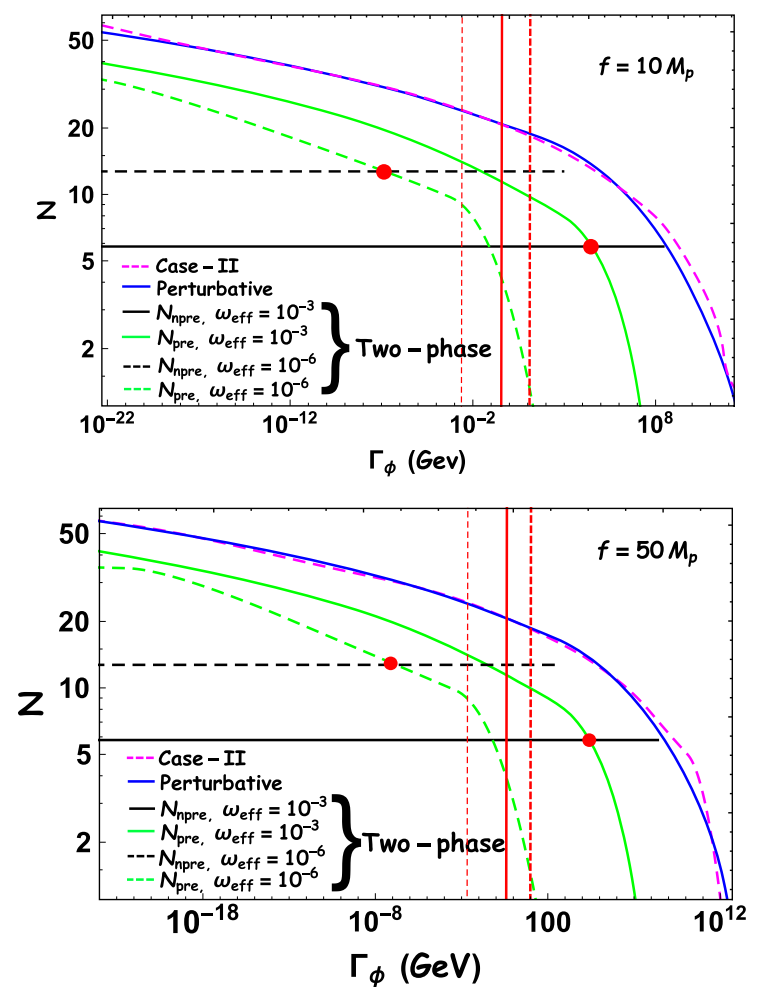

and $T_{\mathrm{re}}^{\mathrm{cri}} \simeq\left(9 \times 10^{10}, 1.9 \times 10^{5}\right) \mathrm{GeV}$. For both the cases, the effective equations of state are taken to be $\omega_{\text {eff }}=\left(10^{-3}, 10^{-6}\right)$. On the other hand, the theoretical value of the critical inflaton decay constants for three different interacting reheating models are calculated to be $\Gamma_{\phi}^{\text {cri }}($ model $)=\left(2.8 \times 10^{-3}, 0.45,10.8\right) \mathrm{GeV}$ for $f=10 M_{P}$ and $\Gamma_{\phi}^{\text {cri }}($ model $)=\left(3.2 \times 10^{-3}, 0.51,12.3\right) \mathrm{GeV}$ for $f=50 M_{P}$. Those values of decay constants are determined from Eqs. (30), (26), and (34) accordingly. Let us point out again that the first two values correspond to inflaton decaying into the scalar particle, and the third one corresponds to decaying into a pair of fermionic particles. Here again, from the left panel in Fig. 5, one concludes that if the Universe undergoes two-phase reheating, considering the specific interaction during reheating, the initial $\omega_{\text {eff }}$ during phase I must lie within $\left(10^{-3}, 10^{-6}\right)$.

The lower limit of $n_{s}$ has been set by the minimum possible reheating temperature due to the $\mathrm{BBN}$ constraint. With increasing the spectral index from its minimum value $n_{s}^{\min }$ along with decay width, the perturbative $e$-folding number $N_{\text {pre }}$ depreciates toward zero, and the total $e$-folding number $N_{\text {re }}$ approaches toward $N_{\text {npre }}$, which is identified as the point of $T_{\mathrm{re}}^{\max }$ and $n_{s}^{\max }$. Following the discussion of the chaotic inflation model, in Table I, we provide a possible limiting value of the inflationary parameters $\left(n_{s}^{\min }, n_{s}^{\max }, N_{k}^{\max }\right)$ parameters for three different reheating scenarios. These limiting values, in turn, will
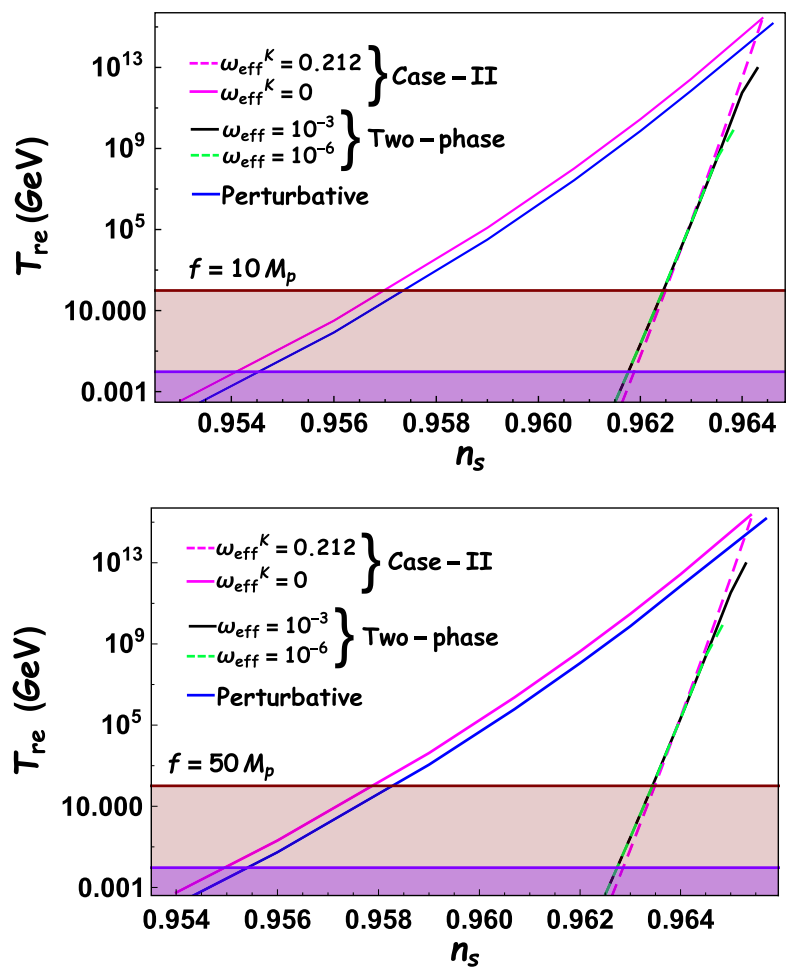

FIG. 5. All plots are same as in the previous Fig. 4. The main difference is that, here, we have plotted for the natural inflation model for $f=(10,50) M_{p}$. 
TABLE I. Reheating models and their associated bound on inflationary parameters (axion inflation).

\begin{tabular}{|c|c|c|c|c|c|}
\hline \multicolumn{6}{|c|}{$f=10 M_{p}$} \\
\hline & \multicolumn{2}{|c|}{ Case I (two-phase) } & \multicolumn{2}{|c|}{ Case II } & \multirow{2}{*}{$\begin{array}{c}\text { Case III (perturbative) } \\
\omega_{\phi}=0\end{array}$} \\
\hline Inflationary parameter & $\omega_{\text {eff }}=10^{-3}$ & $\omega_{\text {eff }}=10^{-6}$ & $\omega_{\mathrm{eff}}^{K}=0$ & $\omega_{\mathrm{eff}}^{K}=0.212$ & \\
\hline$n_{s}^{\min }$ & 0.9618 & 0.9618 & 0.9541 & 0.9619 & 0.9545 \\
\hline$n_{s}^{\max }$ & 0.9643 & 0.9639 & 0.9644 & 0.9644 & 0.9646 \\
\hline$N_{k}^{\max }$ & 57.06 & 56.39 & 57.23 & 57.23 & 57.58 \\
\hline \multicolumn{6}{|c|}{$f=50 M_{p}$} \\
\hline & \multicolumn{2}{|c|}{ Case I (two-phase) } & \multicolumn{2}{|c|}{ Case II } & Case III (perturbative) \\
\hline Inflationary parameter & $\omega_{\text {eff }}=10^{-3}$ & $\omega_{\text {eff }}=10^{-6}$ & $\omega_{\mathrm{eff}}^{K}=0$ & $\omega_{\mathrm{eff}}^{K}=0.212$ & $\omega_{\phi}=0$ \\
\hline$n_{s}^{\min }$ & 0.96275 & 0.96275 & 0.9549 & 0.9629 & 0.9554 \\
\hline$n_{s}^{\max }$ & 0.9653 & 0.9649 & 0.9654 & 0.9654 & 0.9657 \\
\hline$N_{k}^{\max }$ & 57.14 & 56.48 & 57.31 & 57.31 & 57.81 \\
\hline
\end{tabular}

restrict the possible values of reheating parameters. Therefore, the more we decrease the error of the inflationary parameter more precisely, we will be able to fix the reheating parameters.

\section{D. $\alpha$-attractor model [75]}

This is a new class of model that unifies many of the existing inflationary models in a single framework and was first proposed in Ref. [75]. This is currently the most favored model from the observational point of view. A class of $\alpha$-attractor potential, known as the $E$ model, is given as

$$
V(\phi)=\Lambda^{4}\left[1-e^{-\sqrt{2 / 3 \alpha}\left(\phi / M_{p}\right)}\right]^{2 n}
$$

where the mass scale $\Lambda$ is fixed from the CMB power spectrum. An important feature of this class of potential is a large plateau region for the large field value. It also predicts a very low value of the scalar-to-tensor ratio for different $n$ and $\alpha$. However, it is worth noting that, for $n=1, \alpha=1$, this model reduces to the Higgs-Starobinsky model [76]. So the form of the potential for the Higgs-Starobinsky model is as follows:

$$
V(\phi)=\beta\left(1-e^{-\sqrt{2 / 3}\left(\phi / M_{p}\right)}\right)^{2}
$$

where the dimension full parameter $\beta$ takes the following forms:

$$
\beta_{S}=\frac{1}{4 \alpha}, \quad \beta_{H}=\frac{\lambda M_{p}^{4}}{\xi^{2}} .
$$

Prefixes $S$ and $H$ stand for Starobinsky and Higgs model, respectively. The aforementioned coupling parameters appear in the noncanonical Lagrangian as follows:

$$
\begin{aligned}
\mathcal{L}_{S} & =\frac{M_{p}^{2}}{2} R_{J}\left(1+\alpha R_{J}\right)+\cdots, \\
\mathcal{L}_{H} & =\frac{M_{p}^{2}}{2} R_{J}+\frac{2 \xi R_{J}}{M_{p}^{2}} h^{2}-\frac{1}{2} \partial_{\mu} h \partial^{\mu} h-\frac{\lambda}{4} h^{4}+\cdots,
\end{aligned}
$$

where $R_{J}$ is the Ricci scalar in the Jordan frame. For the Higgs inflation model, one assumes $\left(\xi>1, h / M_{p}>1\right)$ during inflation. The inflaton degrees of freedom $\phi$ in Eq. (79) are expressed as

$$
\phi_{S}=\sqrt{\frac{2}{3}} \ln \left(1+2 \alpha R_{J}\right), \quad \phi_{H}=\sqrt{\frac{2}{3}} \ln \left(1+\frac{\xi h^{2}}{M_{p}^{2}}\right),
$$

in units of $M_{p}$. For our purpose, we have taken two values of $\alpha(1,100)$ with $n=1$ and compare their outcomes.

\section{Initial conditions for phase I}

Initial conditions to solve the differential equations for the effective nonperturbative era in the context of present model can be expressed as

$$
\begin{aligned}
& \Phi(A=1)=\frac{3}{2} \Lambda^{4}\left(\frac{2 n}{2 n+\sqrt{3 \alpha}}\right)^{2 n}, \\
& R(A=1)=\frac{3\left(\omega_{\mathrm{eff}}-\omega_{\phi}\right)}{1-3 \omega_{\mathrm{eff}}} \Phi(A=1),
\end{aligned}
$$

where

$$
\begin{aligned}
\Lambda= & M_{p}\left(\frac{3 \pi^{2} r A_{s}}{2}\right) \\
& \times\left[\frac{2 n(1+2 n)+\sqrt{4 n^{2}+6 \alpha(1+n)\left(1-n_{s}\right)}}{4 n(1+n)}\right]^{n / 2} .
\end{aligned}
$$

The inflationary $e$-folding number $N_{k}$ and tensor-toscalar ratio $r_{k}$ can be written in terms of the inflationary spectral index $\left(n_{s}\right)$ as 


$$
\begin{aligned}
N_{k} & =\frac{3 \alpha}{4 n}\left[e^{\sqrt{2 / 3 \alpha}\left(\Phi_{k} / M_{p}\right)}-e^{\sqrt{2 / 3 \alpha}\left(\Phi_{\mathrm{end}} / M_{p}\right)}-\sqrt{\frac{2}{3 \alpha}} \frac{\left(\Phi_{k}-\Phi_{\mathrm{end}}\right)}{M_{p}}\right], \\
r_{k} & =\frac{64 n^{2}}{3 \alpha\left(e^{\sqrt{2 / 3 \alpha}\left(\Phi_{k} / M_{p}\right)}-1\right)^{2}} .
\end{aligned}
$$

Furthermore, the initial conditions to solve the Boltzmann equations for different energy components during the perturbative epoch are determined by the spectral index at the ending point of the effective dynamics, $A_{\text {npre }}$. All the initial conditions for phase II will be the same as before, provided in Eq. (73).

\section{Observations}

We have chosen two sample values of $\alpha=(1,100)$. With these two values, the model-dependent critical values of the inflaton decay constant assume $\Gamma_{\phi}^{\text {cri }}($ model $)=$ $(0.069,5.03,260.3)$ and $(0.01,1.6,42.2) \mathrm{GeV}$ for three different kinds of the decay processes, whereas our numerical analysis predicts the critical decay constant to be $\Gamma_{\phi}^{\text {cri }}=\left(3.44 \times 10^{3}, 1.37 \times 10^{-7}\right) \mathrm{GeV}$ for $\alpha=1$ and $\Gamma_{\phi}^{\mathrm{cri}}=\left(1.27 \times 10^{4}, 3.90 \times 10^{-6}\right) \mathrm{GeV}$ for $\alpha=100$ with $\omega_{\text {eff }}=\left(10^{-3}, 10^{-6}\right)$, respectively. Within these values, all the model-dependent critical decay constants must lie (see Fig. 6). In addition to that, the reheating temperature connected with the critical value of the inflaton decay constant turns out to be $T_{\mathrm{re}}^{\text {cri }} \simeq\left(2.3 \times 10^{5}, 3.5 \times 10^{10}\right) \mathrm{GeV}$ for $\alpha=1$ and for $\alpha=100, T_{\mathrm{re}}^{\text {cri }} \simeq\left(1.2 \times 10^{6}, 7.2 \times\right.$ $\left.10^{10}\right) \mathrm{GeV}$ with $\omega_{\text {eff }}=\left(10^{-6}, 10^{-3}\right)$ accordingly. Similar to the other inflation model discussed above, in Table II the possible constraints on the inflationary parameters can be obtained.

\section{E. Minimal plateau inflation model [77]}

The minimal plateau inflationary model is a nonpolynomial modification of the power-law chaotic potential. The potential for this inflation is given by

$$
V_{\min }=\Lambda \frac{m^{4-n} \phi^{n}}{1+\left(\frac{\phi}{\phi_{*}}\right)^{n}}
$$
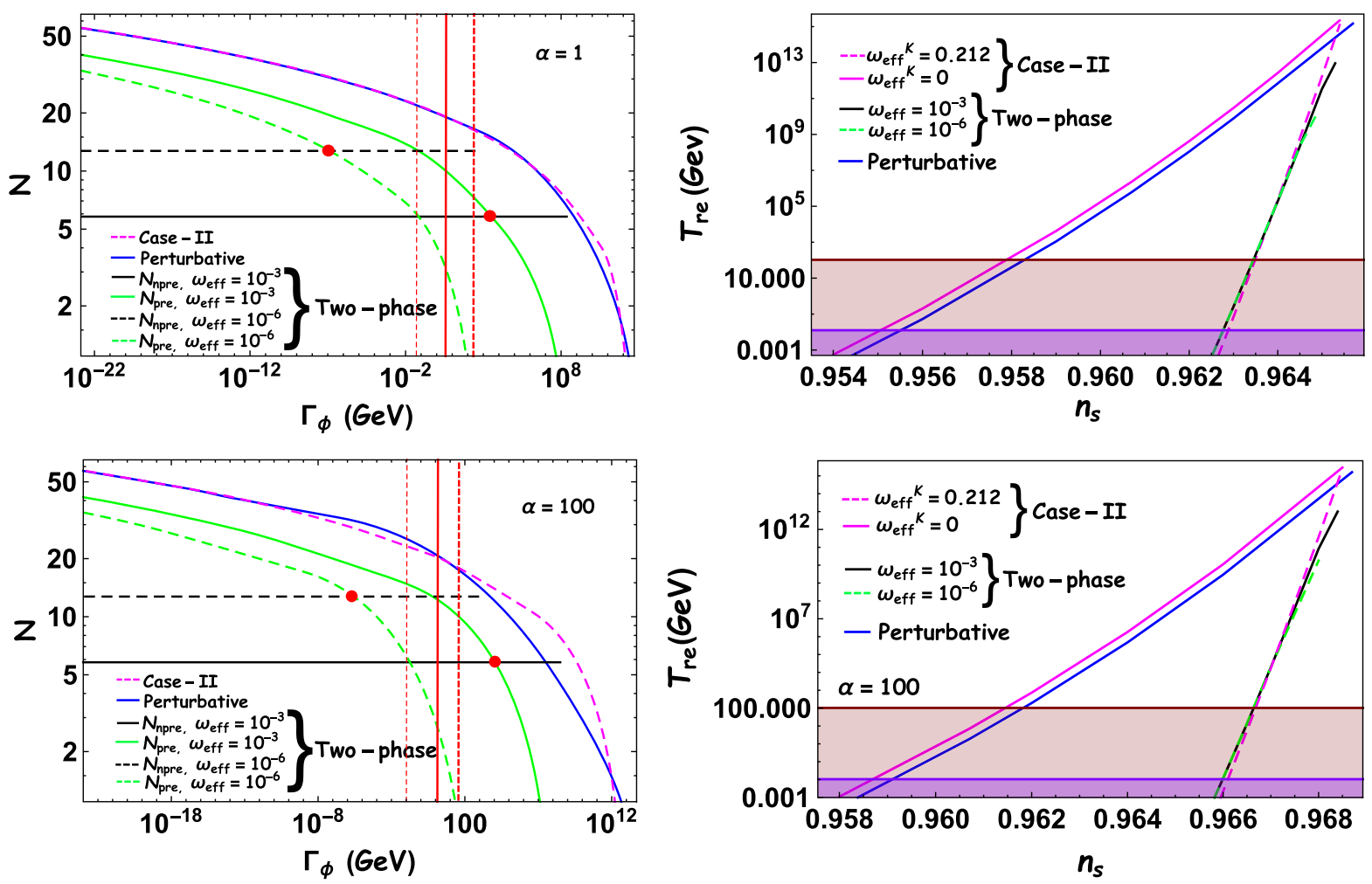

FIG. 6. All plots are same as in the previous Fig. 4. The main difference is that, here, we have plotted for the $\alpha$-attractor model for $\alpha=(1,100)$ with $n=1$. However, the plot for $\alpha=1$ and $n=1$ is for the Higgs-Starobinsky model. 
TABLE II. Reheating models and their associated bound on inflationary parameters ( $\alpha$-attractor model).

\begin{tabular}{|c|c|c|c|c|c|}
\hline \multicolumn{6}{|c|}{ Higgs-Starobinsky model $(\alpha=1)$} \\
\hline \multirow[b]{2}{*}{ Inflationary parameter } & \multicolumn{2}{|c|}{ Case I (two-phase) } & \multicolumn{2}{|c|}{ Case II } & \multirow{2}{*}{$\begin{array}{c}\text { Case III (perturbative) } \\
\omega_{\phi}=0\end{array}$} \\
\hline & $\omega_{\text {eff }}=10^{-3}$ & $\omega_{\mathrm{eff}}=10^{-6}$ & $\overline{\omega_{\text {eff }}^{K}}=0$ & $\omega_{\text {eff }}^{K}=0.212$ & \\
\hline$n_{s}^{\min }$ & 0.9626 & 0.9626 & 0.9548 & 0.9628 & 0.9552 \\
\hline$n_{s}^{\max }$ & 0.9652 & 0.9648 & 0.9653 & 0.9653 & 0.9656 \\
\hline$N_{k}^{\max }$ & 55.36 & 54.72 & 55.52 & 55.52 & 56.02 \\
\hline \multicolumn{6}{|c|}{$\alpha=100$} \\
\hline & \multicolumn{2}{|c|}{ Case I (two-phase) } & \multicolumn{2}{|c|}{ Case II } & Case III (perturbative) \\
\hline Inflationary parameter & $\omega_{\text {eff }}=10^{-3}$ & $\omega_{\text {eff }}=10^{-6}$ & $\omega_{\mathrm{eff}}^{K}=0$ & $\omega_{\mathrm{eff}}^{K}=0.212$ & $\omega_{\phi}=0$ \\
\hline$n_{s}^{\min }$ & 0.966 & 0.966 & 0.9587 & 0.9661 & 0.959 \\
\hline$n_{s}^{\max }$ & 0.9684 & 0.968 & 0.9685 & 0.9685 & 0.9657 \\
\hline$N_{k}^{\max }$ & 56.73 & 56.03 & 56.91 & 56.91 & 57.27 \\
\hline
\end{tabular}

where $n, \Lambda$, and $m$ have the same role as in the power-law chaotic inflation model and their values are fixed from WMAP normalization [78]. Only even values of $n$ are taken, as in the case of the chaotic inflation model. The new scale of $\phi_{*}$ controls the shape of the potential. For a wide range of $\phi_{*}$, this model predicts lower values of scalar-totensor ratio for different values of $n$ and satisfies the latest Planck data [3]. For numerical purpose, we consider $\phi_{*}=$ $(0.001,0.1) M_{p}$ with $n=2$.

\section{Initial conditions for phase I}

The initial conditions are set as

$\Phi(A=1)=\frac{3}{2} \frac{V_{\mathrm{end}}}{m_{\phi}^{4}}, \quad R(A=1)=\frac{3\left(\omega_{\mathrm{eff}}-\omega_{\phi}\right)}{1-3 \omega_{\mathrm{eff}}} \Phi(A=1)$,

where

$$
\begin{aligned}
V_{\text {end }} & =\frac{m^{4-n} \phi_{\text {end }}^{n}}{1+\left(\frac{\phi_{\text {end }}}{\phi_{*}}\right)^{n}} \\
m & =\left(\frac{3 \pi^{2} M_{p}^{4} r_{k} A_{s}}{2 \Lambda \phi_{k}^{n}}\left(1+\left(\frac{\phi_{k}}{\phi_{*}}\right)^{n}\right)\right)^{1 /(4-n)} .
\end{aligned}
$$

We set $\Lambda=1$, except for $n=4$. Constraining the parameter $\Lambda$ for $n=4$ has been studied in the context of minimal Higgs inflation in Ref. [79]. The inflationary parameters $N_{k}$ and $r_{k}$ can be written as

$r_{k}=\frac{8 M_{p}^{2} n^{2}}{\phi^{2}\left(1+\left(\frac{\phi}{\phi_{*}}\right)^{n}\right)^{2}}, \quad N_{k}=\int_{\phi_{k}}^{\phi_{\text {end }}}-\frac{\phi\left(\phi_{*}^{n}+\phi^{n}\right)}{n M_{p}^{2} \phi_{*}^{n}} d \phi$.

Similar to the other inflationary models, the initial condition of the second phase boundary condition is set at the normalized scale factor at $A_{\text {npre }}$ through Eq. (73). As we mentioned earlier, our main intention is to see the modification in reheating parameters $\left(T_{\text {re }}, N_{\text {re }}\right)$ in comparison with the usual analysis.

\section{Observations}

The important results of the minimal plateau inflationary model are depicted in Fig. 7. In this model, the values of $\Gamma_{\phi}^{\text {cri }}($ model $)$ for three different decay processes assume $\Gamma_{\phi}^{\text {theo }}=(0.7,34.2,2749.2) \mathrm{GeV}$ for $\phi_{*}=0.01$ and $\left(15.3,231.7,5.8 \times 10^{4}\right) \mathrm{GeV}$ for $\phi_{*}=0.001 M_{p}$. As usual, those values are obtained from Eqs. (30), (26), and (34) with $\phi_{*}=(0.01,0.001) M_{p}$ accordingly. On the other hand, our numerical analysis estimates the value of $\Gamma_{\phi}^{\text {cri }}=$ $\left(2.3 \times 10^{3}, 4.8 \times 10^{-7}\right) \mathrm{GeV}$ for $\phi_{*}=0.01 M_{p}$ and $\Gamma_{\phi}^{\mathrm{cri}}=$ $\left(394.7,2.7 \times 10^{-8}\right) \mathrm{GeV}$ for $\phi_{*}=0.001 M_{p}$. As discussed for other inflationary scenarios, for each model parameter value of $\phi_{*}$, two bracketed values of $\Gamma_{\phi}^{\mathrm{cri}}$ are calculated for $\omega_{\text {eff }}=\left(10^{-3}, 10^{-6}\right)$, respectively. The reheating temperature linked with the decay width $\Gamma_{\phi}^{\text {cri }}$ can be found to be $T_{\mathrm{re}}^{\mathrm{cri}} \simeq\left(2.80 \times 10^{5}, 2.25 \times 10^{10}\right) \mathrm{GeV}$ considering $\phi_{*}=0.01 M_{p}$, and, for $\phi_{*}=0.001 M_{p}, T_{\mathrm{re}}^{\mathrm{cri}} \simeq(5.80 \times$ $\left.10^{4}, 8.76 \times 10^{9}\right) \mathrm{GeV}$ with $\omega_{\text {eff }}=\left(10^{-6}, 10^{-3}\right)$ accordingly. Interestingly, for this minimal inflation scenario, a specific choice of $\phi_{*}=0.01 M_{p}, \omega_{\mathrm{eff}}=10^{-3}, \Gamma_{\phi}^{\mathrm{cri}}$ approximately matches with that of $\Gamma_{\phi}^{\mathrm{cri}}($ model $)$ for a specific reheating scenario when inflaton decaying into a pair of fermionic particles with the interaction $\phi \bar{\psi} \psi$. Similarly, for $\phi_{*}=0.001 M_{p}, \omega_{\text {eff }}=10^{-3}$, we found $\Gamma_{\phi}^{\mathrm{cri}} \simeq \Gamma_{\phi}^{\mathrm{cri}}($ model $)$ when reheating dynamics is governed by the inflaton decaying into a pair of scalar particles with the interaction $\phi \chi^{2}$. Associated with the reheating temperature, the bound on the inflationary parameters are given in Table III. 

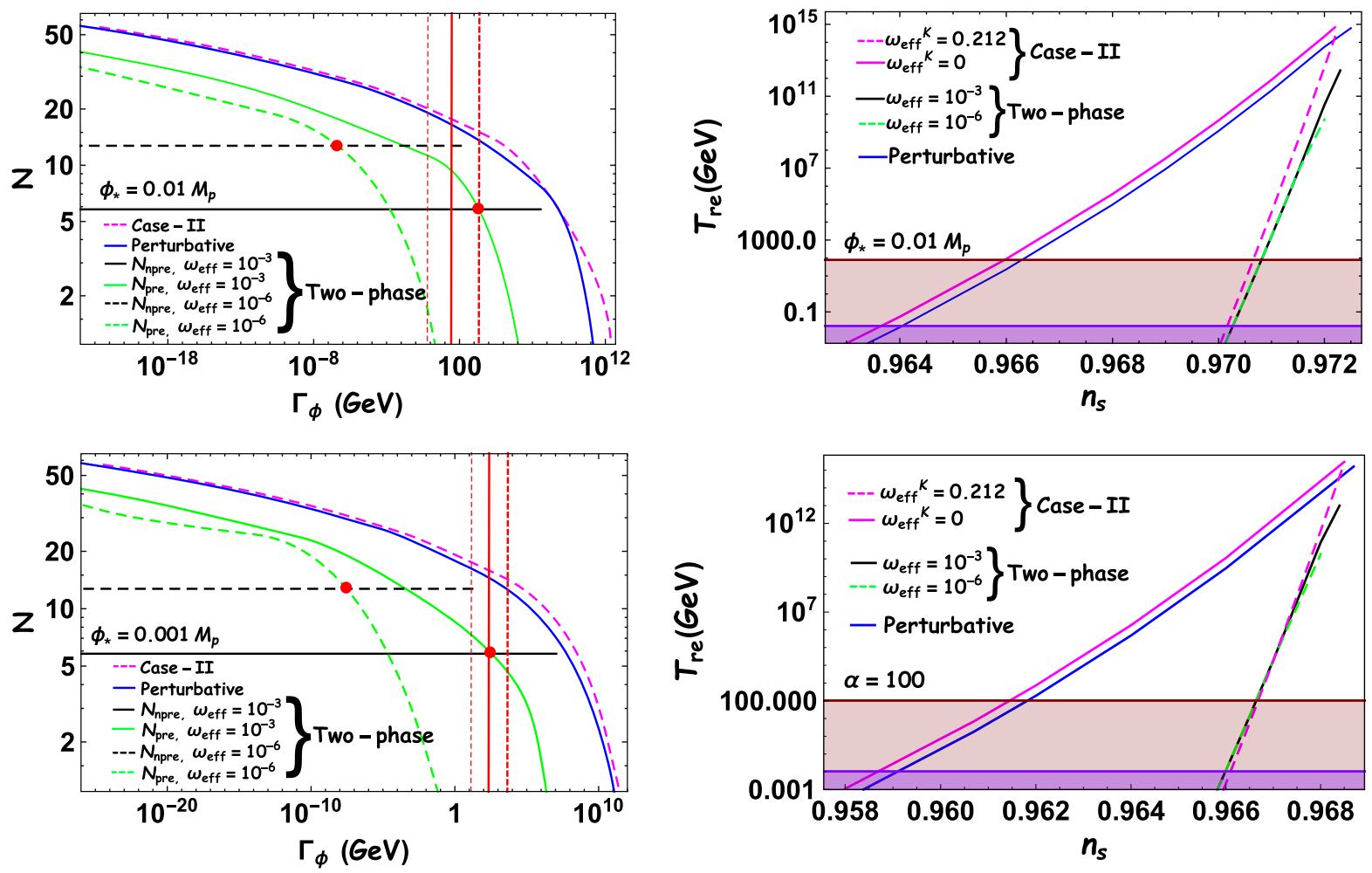

FIG. 7. All plots are the same as in the previous Fig. 4. The main difference is that, here, we have plotted for the minimal inflation model with $\phi_{*}=(0.01,0.001) M_{p}, n=2$.

\section{CONSTRAINING THE INFLATON COUPLING PARAMETERS}

So far, we have discussed mainly understanding the reheating parameters and their constraints from reheating. In this section, we qualitatively translate those results into constraints on coupling parameters $\left(\tilde{g}=g / m_{\phi}, y, h\right)$ corresponding to specific inflaton-scalar interactions $\tilde{g} m_{\phi} \phi \chi^{2}$, $y \phi \chi^{3}$ and inflaton-fermion interaction $h \phi \bar{\psi} \psi$, respectively.
So far, our analysis was independent of the specific inflaton interaction model. Therefore, the inflaton decay width was a free parameter with one-to-one correspondence with the reheating temperature. Constraining reheating models is very challenging from the perspective of its observational limitations. Therefore, indirect constraints on the inflaton coupling parameters through reheating dynamics would be significant from the model building point of view. The reheating temperature directly estimates the allowed ranges

TABLE III. Reheating models and their associated bound on inflationary parameters (minimal plateau model).

\begin{tabular}{|c|c|c|c|c|c|}
\hline \multicolumn{6}{|c|}{$\phi_{*}=0.01 M_{p}$} \\
\hline \multirow[b]{2}{*}{ Inflationary parameter } & \multicolumn{2}{|c|}{ Case I (two-phase) } & \multicolumn{2}{|c|}{ Case II } & \multirow{2}{*}{$\frac{\text { Case III (perturbative) }}{\omega_{\phi}=0}$} \\
\hline & $\omega_{\text {eff }}=10^{-3}$ & $\omega_{\text {eff }}=10^{-6}$ & $\omega_{\mathrm{eff}}^{K}=0$ & $\omega_{\mathrm{eff}}^{K}=0.212$ & \\
\hline$n_{s}^{\min }$ & 0.9703 & 0.9703 & 0.9637 & 0.9702 & 0.9640 \\
\hline$n_{s}^{\max }$ & 0.9723 & 0.972 & 0.9722 & 0.9722 & 0.9725 \\
\hline$N_{k}^{\max }$ & 54.16 & 53.58 & 53.96 & 53.96 & 54.55 \\
\hline \multicolumn{6}{|c|}{$\phi_{*}=0.001 M_{p}$} \\
\hline & \multicolumn{2}{|c|}{ Case I (two-phase) } & \multicolumn{2}{|c|}{ Case II } & Case III (perturbative) \\
\hline Inflationary parameter & $\omega_{\text {eff }}=10^{-3}$ & $\omega_{\text {eff }}=10^{-6}$ & $\omega_{\mathrm{eff}}^{K}=0$ & $\omega_{\mathrm{eff}}^{K}=0.212$ & $\omega_{\phi}=0$ \\
\hline$n_{s}^{\min }$ & 0.9700 & 0.9700 & 0.9632 & 0.9698 & 0.9636 \\
\hline$n_{s}^{\max }$ & 0.9720 & 0.9717 & 0.9719 & 0.9719 & 0.9722 \\
\hline$N_{k}^{\max }$ & 53.57 & 53.00 & 53.38 & 53.38 & 53.96 \\
\hline
\end{tabular}



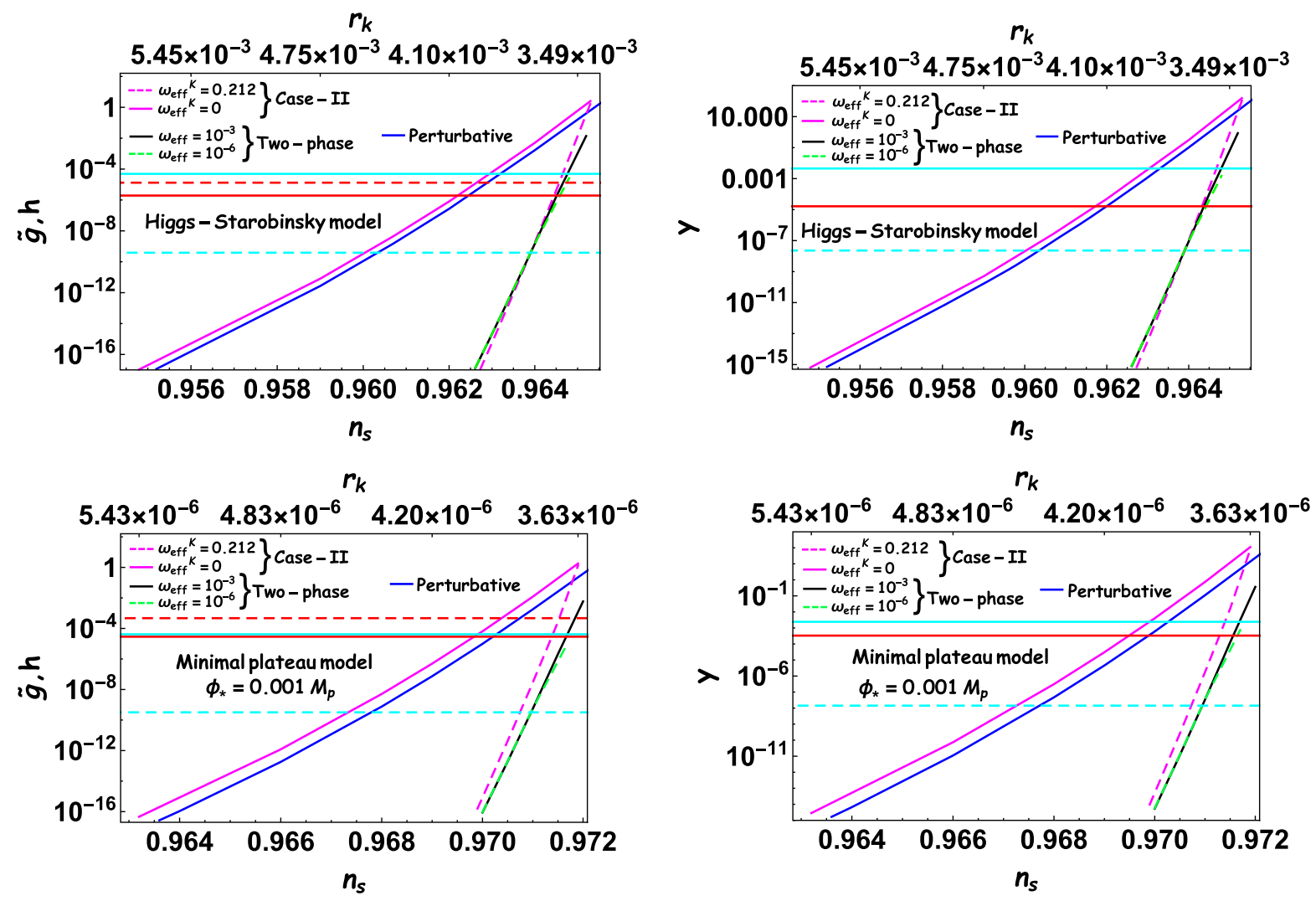

FIG. 8. We have plotted the spectral index dependence of the dimensionless coupling constant $\tilde{g}=\frac{g}{m_{\phi}}$ with $g \phi \chi^{2}$ interaction, $y$ with three-body $y \phi \chi^{3}$ interaction, and Yukawa coupling with $y \phi \psi \bar{\psi}$ interaction. The upper two plots are for the Higgs-Starobinsky inflation model, and the lower two plots are for the minimal plateau inflation model with $\phi_{*}=0.001 M_{p}, n=2$. The solid and dashed pink lines correspond to the usual reheating dynamics given by Kamionkowski et al. [11] for $\omega_{\text {eff }}^{K}=(0,0.212)$, respectively. The solid blue line indicate the results for perturbative analysis. The results for our developed two-phase reheating mechanism are represented by the solid black line and dashed green line for $\omega_{\text {eff }}=\left(10^{-3}, 10^{-6}\right)$ accordingly. In the first and third plots, the solid and dashed red lines imply the transition point from parametric resonance to perturbative dynamics for two different kinds of interaction, $g \phi \chi^{2}$ and $y \phi \psi \bar{\psi}$, respectively, measured from theoretical constraints provided by Eqs. (25) and (33). Similarly, in the second and fourth plots, the solid red line corresponds to the three-body $y \phi \chi^{3}$ interaction. Additionally, the solid and dashed sky blue lines indicate the coupling constant at the intersection points of the $e$-folding numbers, $N_{\text {npre }}$ and $N_{\text {pre }}$, respectively, above which value the effective dynamics start dominating over perturbative dynamics for $\omega_{\text {eff }}=\left(10^{-3}, 10^{-6}\right)$, respectively. All the plots are drawn within the minimum and maximum values of the spectral index. The minimum value of the spectral index $\left(n_{s}^{\mathrm{min}}\right)$ corresponds to $T_{\mathrm{re}} \approx 10^{-2} \mathrm{GeV}$ and for maximum values of spectral index $\left(n_{s}^{\max }\right), N_{\text {re }} \approx N_{\text {npre }}$ in our analysis and $N_{\text {re }} \rightarrow 0$ in conventional reheating dynamics.

of dimensionless coupling parameter via the inflaton decay constant $\Gamma_{\phi}$. In this section, for illustration only, we consider two observationally viable inflationary models: Higgs-Starobinsky and minimal plateau models with $n=2$, which are consistent with the current observational bound on $r<0.064$ [3].

\section{A. Bounds on couplings}

The constraints on different coupling constants are shown in Fig. 8. Plots show how the dimensionless coupling parameters $\tilde{g}, h$, and $y$ are intimately linked with CMB anisotropy via the inflationary observables such as $\left(n_{s}, r_{k}\right)$ for different types of reheating dynamics. The mapping $T_{\mathrm{re}} \rightarrow \Gamma_{\phi} \rightarrow(\tilde{g}, y, h)$ directly follows from Eqs. (22), (28), (32), and (52). From these equations, we obtain the constraints on the coupling parameters with respect to the inflationary parameters. Any realistic scenario of reheating should include all possible inflaton coupling based on underlying symmetry. Therefore, the assumption of a specific inflaton coupling's contribution to be the dominant one throughout the entire reheating period may not be relevant. Hence, a more pragmatic approach would be to construct particle physics motivated models which we left for our future study. However, as a toy model analysis, the present study may guide us in building scenarios that include all the standard model fields. Nevertheless, based on our reheating discussions so far, we compare the constraints for all the cases. To this end, let us point out that, in terms of mathematical expression, the decay widths $\Gamma_{\phi}$ associated with the coupling parameters $\tilde{g}$ 
TABLE IV. Reheating models and their associated bound on coupling parameters.

Higgs-Starobinsky model

\begin{tabular}{|c|c|c|c|c|c|}
\hline \multirow[b]{2}{*}{ Coupling parameter } & \multicolumn{2}{|c|}{ Case I (two-phase) } & \multicolumn{2}{|c|}{ Case II } & \multirow{2}{*}{$\frac{\text { Case III (perturbative) }}{\omega_{\phi}=0}$} \\
\hline & $\omega_{\mathrm{eff}}=10^{-3}$ & $\omega_{\text {eff }}=10^{-6}$ & $\omega_{\mathrm{eff}}^{K}=0$ & $\omega_{\mathrm{eff}}^{K}=0.212$ & \\
\hline $\bar{g}_{\min }, h_{\min }$ & $1.31 \times 10^{-17}$ & $1.38 \times 10^{-17}$ & $1.06 \times 10^{-17}$ & $1.13 \times 10^{-17}$ & $1.15 \times 10^{-17}$ \\
\hline$\tilde{g}_{\max }, h_{\max }$ & 0.01 & $2.55 \times 10^{-5}$ & 2.52 & 1.71 & 2.48 \\
\hline$y_{\min }$ & $8.07 \times 10^{-16}$ & $8.47 \times 10^{-16}$ & $6.51 \times 10^{-16}$ & $6.98 \times 10^{-16}$ & $7.10 \times 10^{-16}$ \\
\hline$y_{\max }$ & 0.85 & $1.50 \times 10^{-3}$ & 155.10 & 105.31 & 152.76 \\
\hline
\end{tabular}

\begin{tabular}{|c|c|c|c|c|c|}
\hline \multicolumn{6}{|c|}{ Minimal plateau model $\left(\phi_{*}=0.001 M_{p}\right)$} \\
\hline \multirow[b]{2}{*}{ Coupling parameter } & \multicolumn{2}{|c|}{ Case I (two-phase) } & \multicolumn{2}{|c|}{ Case II } & \multirow{2}{*}{$\begin{array}{c}\text { Case III (perturbative) } \\
\omega_{\phi}=0\end{array}$} \\
\hline & $\omega_{\text {eff }}=10^{-3}$ & $\omega_{\mathrm{eff}}=10^{-6}$ & $\omega_{\mathrm{eff}}^{K}=0$ & $\omega_{\mathrm{eff}}^{K}=0.212$ & \\
\hline$\tilde{g}_{\min }, h_{\min }$ & $8.74 \times 10^{-17}$ & $9.27 \times 10^{-17}$ & $4.62 \times 10^{-17}$ & $1.58 \times 10^{-16}$ & $2.70 \times 10^{-17}$ \\
\hline$\tilde{g}_{\max }, h_{\max }$ & $5.90 \times 10^{-3}$ & $1.19 \times 10^{-5}$ & 1.79 & 1.30 & 1.12 \\
\hline$y_{\min }$ & $5.38 \times 10^{-15}$ & $5.71 \times 10^{-15}$ & $2.84 \times 10^{-15}$ & $9.73 \times 10^{-15}$ & $1.66 \times 10^{-15}$ \\
\hline$y_{\max }$ & 0.36 & $7.37 \times 10^{-4}$ & 110.26 & 80.31 & 68.93 \\
\hline
\end{tabular}

and $h$ are the same. Therefore, for each model under consideration, we have two different figures in the $\left(\tilde{g} / h, n_{s}\right)$ and $\left(y, n_{s}\right)$ space. Given the observation from CMB temperature anisotropy, the coupling parameters for the Higgs-inflation model, which are assumed to be responsible for the entire reheating process, are found to be constrained within $1.31 \times 10^{-17} \leq(\tilde{g}, h) \leq 0.01 \quad$ (solid black curve) and $1.38 \times 10^{-17} \leq(\tilde{g}, h) \leq 2.55 \times 10^{-5}$ (dotted green curve) for two different values of effective equation state $\omega_{\text {eff }}=\left(10^{-3}, 10^{-6}\right)$, respectively, whereas, for the same values of the effective equation of state, the coupling constant $y$ for three-body interaction $\left(y \phi \chi^{3}\right)$ lies within $8.07 \times 10^{-16} \leq y \leq 0.85$ (solid black curve) and $8.47 \times 10^{-16} \leq y \leq 1.50 \times 10^{-3}$ (dotted green curve). It is important but straightforward to note that the largest values of the coupling constant $\tilde{g}_{\max } / h_{\max }=\left(0.01,2.55 \times 10^{-5}\right)$ and $y_{\max }=\left(0.85,1.50 \times 10^{-3}\right)$ correspond to maximum reheating temperature $T_{\mathrm{re}}^{\max }=\left(10^{10}, 10^{13}\right) \mathrm{GeV}$, respectively, reemphasizing the fact that two different limiting values of coupling constants are realizable only in the hightemperature limit for two different $\omega_{\text {eff }}$. All the above estimates are for the two-phase reheating process (case I). For the other two scenarios, the bounds on the coupling constant can be read from Table IV.

The interesting interplay among the inflationary theory parameters and the emergent reheating parameters governed by the CMB anisotropy gives important constraints on the theory itself. Apart from having the maximum possible values of the coupling constants, compatible with $\mathrm{CMB}$ observations, there exists a critical value of the same born out of $\Gamma_{\phi}^{\mathrm{cri}}$, which entails whether the reheating is perturbative or nonperturbative phase dominated. For the minimal plateau model, we found $\left(\tilde{g}_{\text {cri }} \approx 3.46 \times 10^{-5}\right)$ for $\omega_{\text {eff }}=10^{-3}$, which closely matches with the associated perturbative constraints $\tilde{g}_{\text {cri }}$ (model $) \approx 2.38 \times 10^{-5}$. For the Higgs-Starobinsky inflation model, $h_{\text {cri }} \simeq 5.3 \times 10^{-5}$ for $\omega_{\text {eff }}=10^{-3}$, and the associated perturbative constraint for Yukawa interaction is $h_{\text {cri }}($ model $) \simeq 1.33 \times 10^{-5}$. Therefore, we can infer from this observation that our two-phase reheating scenario essentially captures the necessary features of the nonperturbative phase.

So far, we have discussed the reheating dynamics considering inflaton and radiation as the two dynamical components. However, as we all know, dark matter is another important constituent of our present Universe. One of this component's important properties is that its coupling with the standard model fields must be very weak. Apart from this, not much is known about its other fundamental properties, such as charge, mass, and coupling. Experimental searches of this particle are going on across the globe without much success till now. The searches include both directly as well as indirectly observing the properties of this object and, finally, jointly constrain the parameter region. This paper will study the dark matter phenomenology based CMB parameter space following our previous work [36]. We essentially generalize our two-phase reheating formalism and include the dark matter as the third dynamical matter component.

\section{UNIFYING THE DARK SECTOR}

In the previous section, we discussed the two-phase reheating process, where inflaton decays only into radiation. In the present discussion, we add additional dark matter components and discuss the impact on dark matter phenomenology. The assumption is that inflaton decays into radiation and then radiation to dark matter. The methodology of the analysis will be the same as before, except the new additional dynamical equations for dark matter. 


\section{A. Phase I (effective nonperturbative phase)}

The dynamics is governed by

$$
\rho_{t}=\rho_{\phi}+\rho_{R}+\rho_{X}=\rho_{\text {end }}\left(\frac{a_{\text {end }}}{a}\right)^{3\left(1+w_{\text {eff }}\right)},
$$

where the new component $\rho_{X}$ is the energy density of the dark matter particle with mass $M_{X}$ and energy of the dark matter is expressed as $\left\langle E_{X}\right\rangle=\sqrt{M_{X}^{2}+9 T^{2}}$ [80]. $T$ is the temperature. The above equation can be written in differential form as

$\dot{\rho}_{\phi}+\dot{\rho_{R}}+\dot{\rho}_{X}+3 H\left(1+w_{\text {eff }}\right)\left(\rho_{\phi}+\rho_{R}+\rho_{X}\right)=0$.

Besides the above equation, we consider an additional conservation equation characterizing the dynamics of every individual energy components during this phase as

$$
\dot{\rho_{\phi}}+3 H\left(1+w_{\phi}\right) \rho_{\phi}+\dot{\rho_{R}}+4 H \rho_{R}+\dot{\rho}_{X}+3 H \rho_{X}=0 .
$$

To solve the above equations (90) and (91), we need one more condition. We define the ratio of the dark matter and the radiation energy density as $\gamma=\frac{\rho_{X}}{\rho_{R}}$. After combining the above two equations, one finds

$\frac{\rho_{R}}{\rho_{\phi}+\rho_{R}+\rho_{X}}=\frac{\rho_{R}}{\rho_{\phi}+\rho_{R}+\gamma \rho_{R}}=\frac{3\left(\omega_{\phi}-\omega_{\mathrm{eff}}\right)}{3 \omega_{\phi}(1+\gamma)-1}$.

At the initial stage of reheating, the radiation energy density must be very small $\rho_{R} \simeq 0$. Hence, as discussed extensively for the two-component reheating, here also $\omega_{\text {eff }}$ must assume the value very close to the inflaton equation of state $\omega_{\phi}$, at least near the beginning. In terms of dimensionless variable, this phase can be written as

$$
\begin{gathered}
\frac{\Phi^{\prime}}{A^{3 w_{\phi}}}+\frac{R^{\prime}}{A}+\frac{\left\langle E_{X}\right\rangle X^{\prime}}{m_{\phi}}=0, \\
\frac{\Phi^{\prime}}{A^{2+3 w_{\phi}}}+\frac{R}{A^{4}}\left[3\left(1+w_{\text {eff }}\right)-4\right]+\frac{3\left\langle E_{X}\right\rangle X}{m_{\phi} A^{3}} \omega_{\text {eff }}+\frac{R^{\prime}}{A^{3}} \\
+\frac{3 \Phi\left(w_{\text {eff }}-w_{\phi}\right)}{A^{3\left(1+w_{\phi}\right)}}+\frac{X^{\prime}\left\langle E_{X}\right\rangle}{m_{\phi} A^{2}}=0,
\end{gathered}
$$

where the dimensionless dark matter density $X=\frac{\rho_{X}}{\left\langle E_{X}\right\rangle} a^{3}$.

\section{B. Phase II (perturbative phase)}

The subsequent perturbative phase will now be governed by two more parameters related to the dark matter component. Apart from the inflaton equation of state $\omega_{\phi}^{1}$ and the inflaton decay constant $\Gamma_{\phi}$, we have a thermal average of dark matter annihilation cross section $\langle\sigma v\rangle$ and the dark matter mass $M_{X}$. The corresponding dimensionless comoving energy densities' dynamics will be governed by the Boltzmann equation [35]:

$$
\begin{gathered}
\Phi^{\prime}=-c_{1} \frac{A^{1 / 2} \Phi}{\sqrt{\frac{\Phi}{A^{3 \omega_{\phi}^{1}}}+\frac{R}{A}+\frac{X\left\langle E_{X}\right\rangle}{m_{\phi}}}}, \\
R^{\prime}=c_{1} \frac{A^{\left[3\left(1-2 \omega_{\phi}^{1}\right)\right] / 2} \Phi}{\sqrt{\frac{\Phi}{A^{3 \omega_{\phi}^{1}}}+\frac{R}{A}+\frac{X\left\langle E_{X}\right\rangle}{m_{\phi}}}} \\
+c_{2} \frac{A^{-3 / 2}\langle\sigma v\rangle 2\left\langle E_{X}\right\rangle M_{p l}}{\sqrt{\frac{\Phi}{A^{3 \omega_{\phi}^{1}}}+\frac{R}{A}+\frac{X\left\langle E_{X}\right\rangle}{m_{\phi}}}}\left(X^{2}-X_{\mathrm{eq}}^{2}\right), \\
X^{\prime}=-c_{2} \frac{A^{-5 / 2}\langle\sigma v\rangle M_{p l} m_{\phi}}{\sqrt{\frac{\Phi}{A^{3 \omega_{\phi}^{1}}}+\frac{R}{A}+\frac{X\left\langle E_{X}\right\rangle}{m_{\phi}}}}\left(X^{2}-X_{\mathrm{eq}}^{2}\right) .
\end{gathered}
$$

The equilibrium number density of the dark matter particle can be described in terms of the modified Bessel function of the second kind [80]

$$
n_{X}^{\mathrm{eq}}=\frac{g T^{3}}{2 \pi^{2}}\left(\frac{M_{X}}{T}\right)^{2} K_{2}\left(\frac{M_{X}}{T}\right),
$$

and the constants $c_{1}$ and $c_{2}$ are delineated as

$$
c_{1}=\frac{\sqrt{\frac{3}{8 \pi}} M_{p l} \Gamma_{\phi}}{m_{\phi}^{2}}, \quad c_{2}=\sqrt{\frac{3}{8 \pi}} .
$$

We consider fermionic-type dark matter particles with internal degrees of freedom $g$.

\section{Initial conditions}

The general form of the initial conditions during the first phase of reheating (phase I) are

$$
\begin{aligned}
\Phi(1) & =\frac{3}{2} \frac{V\left(\phi_{\mathrm{end}}\right)}{m_{\phi}^{4}}, \quad R(1)=\frac{3\left(\omega_{\mathrm{eff}}-\omega_{\phi}\right)}{1-3 \omega_{\mathrm{eff}}(1+\gamma)} \Phi(1), \\
X(1) & =\frac{\gamma m_{\phi}}{\left\langle E_{X}\right\rangle} R(1) .
\end{aligned}
$$

The initial values of the energy densities for phase II will be set at the normalized scale factor $A_{\text {npre }}$ as

$$
\begin{aligned}
\Phi & =\Phi\left(A_{\text {npre }}\right) ; \quad \frac{R\left(A_{\text {npre }}\right)}{R\left(A_{\text {npre }}\right)+\Phi\left(A_{\text {npre }}\right)+X\left(A_{\text {npre }}\right)} \simeq \frac{1}{2} ; \\
X\left(A_{\text {npre }}\right) & =\frac{\gamma m_{\phi}}{\left\langle E_{X}\right\rangle} R\left(A_{\text {npre }}\right) .
\end{aligned}
$$


As described in detail in Sec. V, radiation energy density is again assumed to be $50 \%$ of the total comoving energy density right after the completion of phase I. Therefore, the dark matter number density will automatically be fixed for a given $\gamma$ value. All the required equations of state for two different phases are assumed to take the same approximate values $\omega_{\phi}^{1} \simeq 0.2$ and $\omega_{\phi} \simeq 0$. The methodology of solving the dynamics will be the same as before except some additional constraints in the dark sector after the end of reheating.

\section{Boundary condition from observations}

The condition for ending the reheating dynamics is set by the following equation:

$$
\begin{aligned}
H^{2} & =\frac{\left.\rho_{\phi}\left(\Gamma_{\phi}, N_{\mathrm{re}}, n_{s}^{k}\right)+\rho_{R}\left(\Gamma_{\phi}, N_{\mathrm{re}}, n_{s}^{k}\right)\right)+\rho_{X}\left(\Gamma_{\phi}, N_{\mathrm{re}}, n_{s}^{k}\right)}{3 M_{p}^{2}} \\
& =\Gamma_{\phi}^{2},
\end{aligned}
$$

supplemented with the observational constraint, namely, the relation the between reheating temperature following from the above equation and present $\mathrm{CMB}$ temperature $T_{0}=2.7 \mathrm{~K} \simeq 2.35 \times 10^{-13} \mathrm{GeV}$ through the relation Eq. (55). A further additional observational constraint is the observed value of the dark matter abundance defined as $\Omega_{X}[81,82]$ :

$\Omega_{X} h^{2}=\left\langle E_{X}\right\rangle \frac{X\left(T_{F}\right) T_{F} A_{F}}{R\left(T_{F}\right) T_{0} m_{\phi}} \Omega_{R} h^{2}=0.1199 \pm 0.0022$,

which is expressed in terms of radiation abundance $\Omega_{R}$ $\left(\Omega_{R} h^{2}=4.3 \times 10^{-5}\right) . T_{F}$ is the temperature at very late time when both dark matter and radiation energy components become stationary. While solving the Boltzmann equations during perturbative reheating (phase II), these conditions will constrain the dark matter parameter $\langle\sigma v\rangle$ (thermal average of the cross section times velocity) for a fixed value of the dark matter mass $M_{X}$ and the inflaton decay constant in terms of the reheating temperature. The detailed analysis only on phase II has already been done in Ref. [35], including the dark matter phenomenology. Nevertheless, we consider only dark matter production via freeze-in mechanism. This mechanism indicates that the dark matter will never reach equilibrium with the thermal bath. This kind of dark matter is known as feebly interacting dark matter [83-94]. We can illustrate that the production of dark matter via freeze-in mechanism through the heavy mediator during reheating is sensitive to the early history of the Universe before the UV-dominated era [95-105].

\section{E. Physical constraints}

Further constraints on the dark matter parameter space will be inherited if one considers various theoretical limits on the scattering cross section. The cross section cannot be arbitrarily large. Perturbative unitarity usually limits the cross section $\langle\sigma v\rangle$ in term of mass, $\langle\sigma v\rangle_{\max }=\frac{8 \pi}{M_{X}^{2}}[106]$, which is shown by pink solid lines in Figs. 9 and 10. On the other hand, we will also have another bound on the cross sections coming from the fact that during reheating dark matter production peaks around the temperature of $T_{*}=\frac{M_{X}}{4}$ [80]. This provides a natural condition on the dark matter number density $n_{X}(T)<n_{X}^{\mathrm{eq}}\left(T_{*}\right)$, as for $T<T_{*}$ the dark matter production would be frozen, and it must be diluted subsequently due to the expansion of the Universe. The aforementioned condition on the dark matter number density sets an upper bound on the cross section $\langle\sigma v\rangle \approx$ $\langle\sigma v\rangle_{T=T_{*}}[80,107]$ :

$$
\begin{aligned}
\langle\sigma v\rangle_{*} \leq & 7 \times 10^{-14}\left(\frac{2}{g}\right)\left(\frac{g_{*}\left(T_{*}\right)}{10}\right)\left(\frac{10}{g_{*}\left(T_{\mathrm{re}}\right)}\right)^{1 / 2}\left(\frac{M_{X}}{10 \mathrm{GeV}}\right) \\
& \times\left(\frac{100 \mathrm{MeV}}{T_{\mathrm{re}}}\right)^{2} \mathrm{GeV}^{-2} .
\end{aligned}
$$
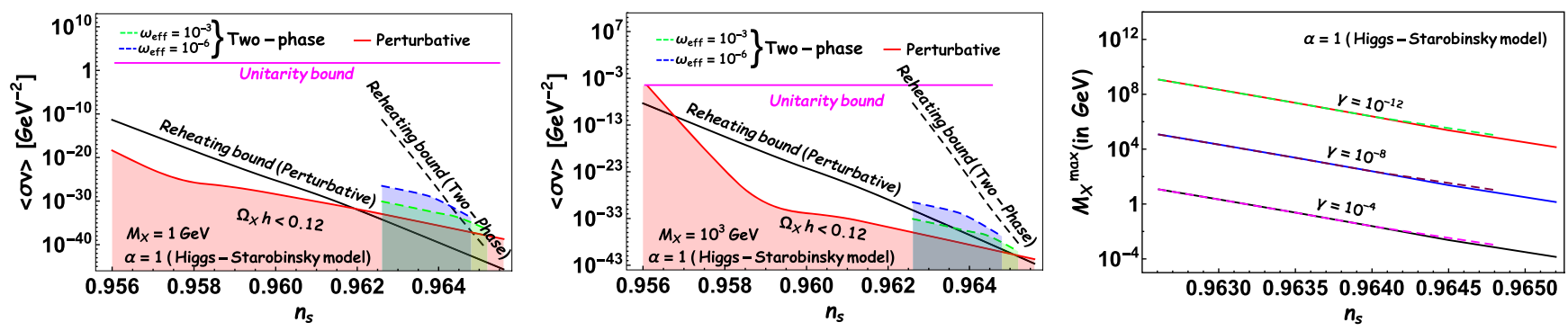

FIG. 9. In the first two plots, we have plotted the contour of $\Omega_{X} h^{2}=0.12$ in the $n_{s}-\langle\sigma v\rangle$ plane with a fixed value of dark matter mass within the minimum and maximum values of the reheating temperature for the Higgs-Starobinsky model. In the case of two-stage reheating, we have chosen a fixed value of $\gamma=10^{-11}$ (ratio of the dark matter energy density to the radiation density) during the first stage of reheating. The allowed parameter space is shown by the shaded region below the contour line. The pink horizontal line corresponds to the unitarity bound. The solid and dashed black lines correspond to the reheating bound for two different reheating processes. On the right-hand side, we have plotted maximum permitted values of dark matter mass as a function of the spectral index for three different values of $\gamma$. Here the solid and dashed lines are for $\omega_{\text {eff }}=\left(10^{-3}, 10^{-6}\right)$, respectively. 

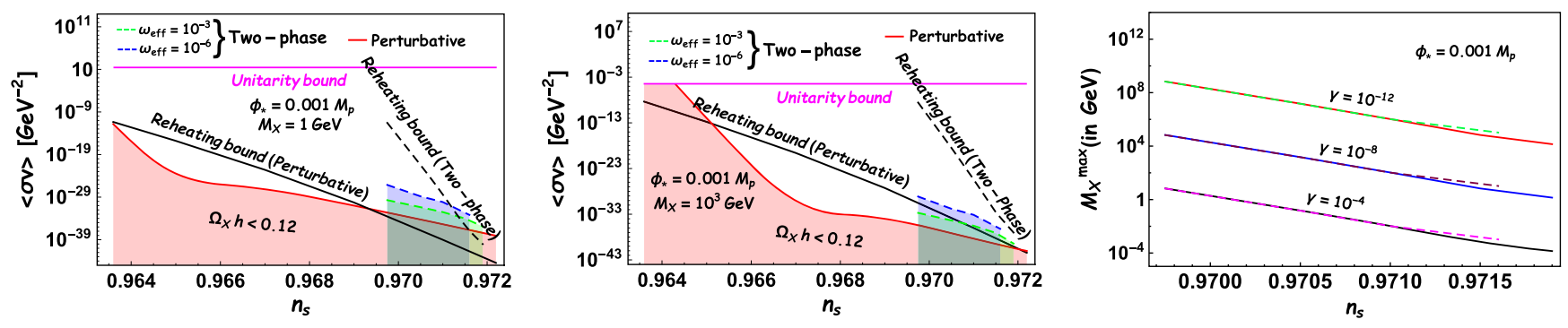

FIG. 10. All plots are same as in the previous Fig. 9. The main difference is that, here, we have plotted for the minimal plateau inflation model with $\phi_{*}=0.001 M_{p}$ and $n=2$.

We call it the reheating bound in the plot. This condition is depicted by black solid lines and black dotted lines for perturbative and two-phase reheating scenarios, respectively, in Figs. 9 and 10. We have shown both of these bounds in the subsequent plots for different inflation model once we fixed the dark matter mass. An interesting observation that can be made from this theoretical constraint is that, given a dark matter mass, the perturbative unitarity bound and the dynamical condition Eq. (104) modify the possible range of allowed $n_{s}$ values obtained from the previous analysis. This can directly shed light on the inflationary model building. Conversely, one can state that, for a given inflationary model, CMB can shed light on the possible nature of the dark matter candidate via the reheating phase.

Nevertheless, unifying the dark sector into a single reheating framework is the primary motivation of this section. The basic philosophy is to look into further constraints on the dark matter parameter space in a more realistic framework of two-phase reheating dynamics and compare with that of the usual perturbative reheating analysis [35]. An important outcome is the constraints due to CMB temperature anisotropy. Particularly, constraints imparted on the dark matter and inflationary parameter space $\left(\langle\sigma v\rangle-n_{s}\right)$ by the CMB anisotropy could enable us to constrain the viable inflationary models through a dark matter observable. Conversely, given a viable inflationary model, $\mathrm{CMB}$ can potentially shed light on the possible properties of dark matter. Keeping this in mind, we study dark matter phenomenology considering two observationally viable inflationary models: Higgs-Starobinsky and minimal plateau models, which are consistent with the current observational bound on $r<0.064$ [3].

\section{F. Higgs-Starobinsky model $[2,108]$ and dark matter phenomenology}

We have already discussed the model in the previous Sec. VII D and the constraints on the reheating parameters $\left(N_{\text {re }}, T_{\text {re }}\right)$ in terms of spectral index $\left(n_{s}\right)$. The inclusion of dark matter does not affect much those parameters. Therefore, the main constraints will be on the thermally averaged cross section times velocity $(\langle\sigma v\rangle)$ and the dark matter mass $M_{X}$. The first two plots of Fig. 9 depict the variation of the annihilation cross section as a function of the spectral index for two different values of dark matter mass $M_{X}=\left(1,10^{3}\right) \mathrm{GeV}$. The range of $n_{s}$ is taken to be within $\left(n_{s}^{\min }, n_{s}^{\max }\right)$ depending upon the model of reheating. For comparison, we include the perturbative reheating scenario [35] as well. Since the viable range of scalar spectral index $n_{s}$ is reduced for the two-phase reheating than that of the perturbative case, consequently the allowed range of $\langle\sigma v\rangle$ is shrunk as shown by green dotted and blue dotted lines. Because of the larger allowed range of $n_{s}$ $\left(n_{s}^{\min } \simeq 0.956, n_{s}^{\max } \simeq 0.9655\right)$, the perturbative reheating [35] widens the allowed range of dark matter annihilation cross section as $2.25 \times 10^{-39} \leq\langle\sigma v\rangle \leq 4.2 \times 10^{-19}$ for $M_{X}=1 \mathrm{GeV}$ and $2.3 \times 10^{-42} \leq\langle\sigma v\rangle \leq 2.51 \times 10^{-5}$ for $M_{X}=10^{3} \mathrm{GeV}$, whereas for the two-phase reheating scenario, for both values of dark matter mass, we can observe the narrower range $\left(n_{s}^{\min } \simeq 0.9626, n_{s}^{\max } \simeq 0.9652\right)$ for $\omega_{\text {eff }}=10^{-3}$ and $\left(n_{s}^{\min } \simeq 0.9626, n_{s}^{\max } \simeq 0.9648\right)$ for $\omega_{\text {eff }}=10^{-6}$. These ranges of $n_{s}$ are well within the $1 \sigma$ range of spectral index, $n_{s}=0.9649 \pm 0.0042$ (68\% C.L., Planck TT, TE, EE + lowE + lensing) from Planck [3]. Detailed constraints on the annihilation cross section for the Higgs inflation model are proved in Table V. Therefore, one can observe the significant differences on the allowed range of dark matter annihilation cross section for two different reheating scenarios (perturbative and two-phase). It is important to note that the dark matter parameter space is constrained by the CMB anisotropy through the inflationary models, or, alternatively, one can state how various dark matter experimental observations can have the potential to constrain the inflationary model through our unified reheating analysis.

The inclusion of dark matter dynamics and the associated theoretical constraints discussed in the previous section has put further limits on the range of $n_{s}$ compatible with the dark matter observation. For example, the perturbative reheating scenario modifies the highest possible value of the spectral index $n_{s}^{\max }$ as $\rightarrow 0.9619$, and for two-phase reheating dynamics $n_{s}^{\max }$ shifts as $(0.9652,0.9648) \rightarrow(0.9645$, $0.9643)$ with $\omega_{\text {eff }}=\left(10^{-3}, 10^{-6}\right)$ accordingly for $M_{X}=$ $1 \mathrm{GeV}$. This modified maximum $n_{s}$ condition leads to the 
TABLE V. Model parameters and associated constraints on the dark matter parameters for different reheating dynamics: HiggsStarobinsky model.

\begin{tabular}{|c|c|c|c|c|c|c|}
\hline \multicolumn{7}{|c|}{$M_{X}=1 \mathrm{GeV}$} \\
\hline \multirow[b]{3}{*}{ Parameters } & & & \multirow{3}{*}{$\begin{array}{c}\text { Perturbative } \\
\omega_{\phi}=0\end{array}$} & \multicolumn{3}{|c|}{ Constraints due to reheating bound } \\
\hline & \multicolumn{2}{|c|}{ Case I (two-phase) } & & \multicolumn{2}{|c|}{ Case I (two-phase) } & \multirow{2}{*}{$\begin{array}{c}\text { perturbative } \\
\omega_{\phi}=0\end{array}$} \\
\hline & $\omega_{\mathrm{eff}}=10^{-3}$ & $\omega_{\mathrm{eff}}=10^{-6}$ & & $\omega_{\mathrm{eff}}=10^{-3}$ & $\omega_{\mathrm{eff}}=10^{-6}$ & \\
\hline$n_{s}^{\min }$ & 0.9626 & 0.9626 & 0.9560 & 0.9626 & 0.9626 & 0.9560 \\
\hline$n_{s}^{\max }$ & 0.9652 & 0.9648 & 0.9655 & 0.9645 & 0.9643 & 0.9619 \\
\hline$\langle\sigma v\rangle_{\min }\left(\mathrm{GeV}^{-2}\right)$ & $3.20 \times 10^{-37}$ & $1.75 \times 10^{-34}$ & $2.25 \times 10^{-39}$ & $1.65 \times 10^{-34}$ & $7.75 \times 10^{-32}$ & $1.66 \times 10^{-32}$ \\
\hline$\langle\sigma v\rangle_{\max }\left(\mathrm{GeV}^{-2}\right)$ & $8.15 \times 10^{-31}$ & $3.10 \times 10^{-27}$ & $4.20 \times 10^{-19}$ & $8.15 \times 10^{-31}$ & $3.10 \times 10^{-27}$ & $4.20 \times 10^{-19}$ \\
\hline \multicolumn{7}{|c|}{$M_{X}=10^{3} \mathrm{GeV}$} \\
\hline & & & & \multicolumn{3}{|c|}{ Constraints due to reheating bound } \\
\hline & \multicolumn{2}{|c|}{ Case I (two-phase) } & Perturbative & \multicolumn{2}{|c|}{ Case I (two-phase) } & Perturbative \\
\hline Parameters & $\omega_{\text {eff }}=10^{-3}$ & $\omega_{\mathrm{eff}}=10^{-6}$ & $\omega_{\phi}=0$ & $\omega_{\mathrm{eff}}=10^{-3}$ & $\omega_{\mathrm{eff}}=10^{-6}$ & $\omega_{\phi}=0$ \\
\hline$n_{s}^{\min }$ & 0.9626 & 0.9626 & 0.9561 & 0.9626 & 0.9626 & 0.9568 \\
\hline$n_{s}^{\max }$ & 0.9652 & 0.9648 & 0.9655 & 0.9652 & 0.9648 & 0.9650 \\
\hline$\langle\sigma v\rangle_{\min }\left(\mathrm{GeV}^{-2}\right)$ & $9.50 \times 10^{-41}$ & $1.60 \times 10^{-37}$ & $2.30 \times 10^{-42}$ & $9.50 \times 10^{-41}$ & $1.60 \times 10^{-37}$ & $1.98 \times 10^{-41}$ \\
\hline$\langle\sigma v\rangle_{\max }\left(\mathrm{GeV}^{-2}\right)$ & $8.10 \times 10^{-34}$ & $3.10 \times 10^{-30}$ & $2.51 \times 10^{-5}$ & $8.10 \times 10^{-34}$ & $3.10 \times 10^{-30}$ & $8.50 \times 10^{-12}$ \\
\hline
\end{tabular}

minimum values of the dark matter cross section $\langle\sigma v\rangle_{\min } \approx 1.66 \times 10^{-32} \mathrm{GeV}^{-2}$ for the perturbative case and $\langle\sigma v\rangle_{\min } \approx\left(1.65 \times 10^{-34}, 7.75 \times 10^{-32}\right) \mathrm{GeV}^{-2}$ for the two-phase reheating case with two different values of $\omega_{\text {eff }}=\left(10^{-3}, 10^{-6}\right)$. For $M_{X}=10^{3} \mathrm{GeV}$, instead, the unitary bound puts stringent constraints on $n_{s}^{\min }$, only for the perturbative process. Furthermore, dynamics during reheating (reheating bound) bounds the cross section within $1.98 \times 10^{-41} \leq\langle\sigma v\rangle \leq 8.5 \times 10^{-12}$ for the perturbative scenario. However, for $M_{X}=10^{3} \mathrm{GeV}$, there is no effect of theoretical constraints on the bound of dark matter annihilation cross section obtained from two-phase reheating analysis.

From the first two plots in Fig. 9, we read the variation of the cross section for two different effective equations of state $\omega_{\text {eff }}$. As we decrease the value of the $\omega_{\text {eff }}$ from $10^{-3} \rightarrow 10^{-6}$, the $e$-folding number $N_{\text {npre }}$, which is nearly independent of the inflationary parameter, changes from $5.8 \rightarrow 12.2$. Another interesting consequence of the phase-I dynamics is the maximum possible value of dark matter mass $M_{X}^{\max }$. To understand the underlying reason behind the origin of $M_{X}^{\max }$, we have computed analytic expressions considering relativistic dark matter. The dark matter number density at the point of freeze-out $n_{X}^{f}$ (see Appendix C) is expressed as

$$
n_{X}^{f} x_{f}^{3}=n_{X}^{\text {in }}+\langle\sigma v\rangle f\left(x_{f}\right),
$$

where expressions of various symbols are given in the Appendix C. $x_{f}=A_{f} / A_{\text {npre }}$ and $A_{f}$ is the normalized scale factor when both comoving dark matter and radiation component become constant. By using the above expression, we can obtain dark matter abundance as

$$
\begin{aligned}
\Omega_{X} h^{2} & \simeq \frac{\left\langle E_{X}\right\rangle_{f} x_{f}^{-3}}{\rho_{R}\left(x_{f}\right)} \frac{T\left(x_{f}\right)}{T_{\text {now }}}\left(n_{X}^{\text {in }}+\langle\sigma v\rangle f\left(x_{f}\right)\right) \Omega_{R} h^{2} \\
& =\frac{\sqrt{M_{X}^{2}+9 T\left(x_{f}\right)^{2}} x_{f}^{-3}}{\rho_{R}\left(x_{f}\right)} \frac{T\left(x_{f}\right)}{T_{\text {now }}}\left(n_{X}^{\text {in }}+\langle\sigma v\rangle f\left(x_{f}\right)\right) \Omega_{R} h^{2} .
\end{aligned}
$$

The above expression indicates that the dark matter abundance increases with increasing dark matter mass. Moreover, at a particular value of the dark matter mass, the dark matter component's initial number density $\left(n_{X}^{\text {in }}\right)$ will also play in the final value of the observed dark matter abundance $\Omega_{X} h^{2}=0.12$. It can be observed from Eq. (106), if $M_{X}>M_{X}^{\max }$, then $\Omega_{X} h^{2}$ always $\geq 0.12$. Therefore, the maximum possible dark matter mass can be obtained from the above equation considering $\Omega_{X} h^{2}=0.12$ as

$$
M_{X}^{\max }=T\left(x_{f}\right) \sqrt{\left(0.12 \frac{\beta}{n_{X}^{\text {in }}} \frac{T_{\text {now }} T\left(x_{f}\right)^{2}}{\Omega_{R} h^{2} x_{f}^{-3}}\right)^{2}-9},
$$

which is dependent on the initial dark matter number density for the phase-II evolution: 


$$
n_{X}^{\text {in }}==\frac{\gamma m_{\phi}}{\left\langle E_{X}\right\rangle} \frac{3\left(\omega_{\text {eff }}-\omega_{\phi}\right)}{1-3 \omega_{\text {eff }}(1+\gamma)} \Phi\left(A_{\text {npre }}\right) A_{\text {npre }}^{-3} m_{\phi}^{3} .
$$

For a given $\gamma$, the initial value of $X$ is clearly set by the value of $\omega_{\text {eff }}$. Therefore, for a given value of phase-I dynamics parameters $\omega_{\text {eff }}$ and $\gamma$, a particular value of the dark matter mass exists above which the present value of the dark matter abundance, $\Omega_{X} h^{2} \approx 0.12$, cannot be achieved irrespective of the cross section values. Equations (107) and (108) illustrate the behavior of $M_{X}^{\max }$, inversely proportional to $\gamma$ as for a fixed value of the spectral index $\left(n_{s}\right)$. From the third plot in Fig. 9, we can also observe the same. Likewise for $n_{s}=0.9635, M_{X}^{\max }=\left(2.38 \times 10^{7}, 2.38 \times 10^{3}\right.$, $0.238) \mathrm{GeV}$ with $\gamma=\left(10^{-12}, 10^{-8}, 10^{-4}\right)$ accordingly once we fixed $\omega_{\text {eff }}=10^{-3}$. Furthermore, from the third plot in Fig. 9, one can observe that $M_{X}^{\max }$ is nearly independent of the choice of $\omega_{\text {eff }}$ except a small deviation as one approaches toward $n_{s}^{\max }$. The straightforward answer could be that $M_{X}^{\max }$ is proportional to the freeze-out radiation temperature $T\left(x_{f}\right)$, which remains invariant with the choice of $\omega_{\text {eff }}$ value.

\section{G. Minimal plateau inflation model [77]}

The details of this model are discussed in Sec. VII E. As was already the case for Higgs inflation, for the minimal inflation model also the reheating parameters such as $\left(T_{\text {re }}, N_{\text {re }}\right)$ will not be modified much because of dark matter dynamics. The reason being, the contribution of dark matter in the background evolution during the reheating phase is insignificant. Throughout this analysis, we consider $\phi_{*}=0.001 M_{p}$, which satisfies the CMB observation.
Another motivation is that, as one increases $\phi^{*}$ value, the models assume a simple power law. Details constraints on the dark matter parameter space can be read off from Fig. 10. Furthermore, the numerical values are provided in Table VI. From the figure, we observed a similar behavior of dark matter annihilation cross section as a function of the spectral index for two different values of $\omega_{\text {eff }}=$ $\left(10^{-3}, 10^{-6}\right)$ with dark matter mass $M_{X}=\left(1,10^{3}\right) \mathrm{GeV}$. For the minimal model, we again identify the maximum allowed values of dark matter mass $M_{X}^{\max }$ followed by Eqs. (107) and (108). Furthermore, for a given value of $n_{s}$, the $M_{X}^{\max }$ turns out to be linearly varying with $\gamma$. Like, for $n_{s}=0.9705, \quad M_{X}^{\max } \simeq\left(1.5 \times 10^{7}, 1.5 \times 10^{3}, 0.15\right) \mathrm{GeV}$ with $\gamma=\left(10^{-12}, 10^{-8}, 10^{-4}\right)$.

\section{SUMMARY AND DISCUSSION}

In this paper, we propose an effective two-phase reheating scenario. After inflation, reheating has been studied extensively in the literature, through either a perturbative or nonperturbative approach. However, it is believed that both approaches independently should not capture the complete picture of the complicated dynamics. In this paper, we, for the first time, study this phase to the best of our knowledge, taking into account both the approaches together motivated by our previous work [37]. However, instead of considering explicit nonperturbative decay of the inflaton field through parametric resonance, we model the initial phase by effective dynamics governed by the standard conservation laws and parametrized by a constant effective equation of state $\left(\omega_{\text {eff }}\right)$. The combined form of conservation laws and the initial condition of the reheating dynamics puts

TABLE VI. Model parameters and associated constraints on the dark matter parameters for different reheating dynamics: minimal plateau model.

\begin{tabular}{|c|c|c|c|c|c|c|}
\hline \multicolumn{7}{|c|}{$\phi_{*}=0.001 M_{p}, M_{X}=1 \mathrm{GeV}$} \\
\hline \multirow[b]{3}{*}{ Parameters } & & & \multirow{3}{*}{$\begin{array}{c}\text { Perturbative } \\
\omega_{\phi}=0\end{array}$} & \multicolumn{3}{|c|}{ Constraints from reheating bound } \\
\hline & \multicolumn{2}{|c|}{ Case I (two-phase) } & & \multicolumn{2}{|c|}{ Case I (two-phase) } & \multirow{2}{*}{$\begin{array}{c}\text { Perturbative } \\
\omega_{\phi}=0\end{array}$} \\
\hline & $\omega_{\mathrm{eff}}=10^{-3}$ & $\omega_{\mathrm{eff}}=10^{-6}$ & & $\omega_{\mathrm{eff}}=10^{-3}$ & $\omega_{\mathrm{eff}}=10^{-6}$ & \\
\hline$n_{s}^{\min }$ & 0.96975 & 0.96975 & 0.9636 & 0.96975 & 0.96975 & 0.9636 \\
\hline$n_{s}^{\max }$ & 0.9719 & 0.9716 & 0.9722 & 0.97136 & 0.9712 & 0.9692 \\
\hline$\langle\sigma v\rangle_{\min }\left(\mathrm{GeV}^{-2}\right)$ & $1.29 \times 10^{-36}$ & $6.80 \times 10^{-34}$ & $8.60 \times 10^{-39}$ & $3.76 \times 10^{-34}$ & $7.31 \times 10^{-32}$ & $2.89 \times 10^{-32}$ \\
\hline$\langle\sigma v\rangle_{\max }\left(\mathrm{GeV}^{-2}\right)$ & $1.95 \times 10^{-30}$ & $7.35 \times 10^{-27}$ & $1.40 \times 10^{-12}$ & $1.95 \times 10^{-30}$ & $7.35 \times 10^{-27}$ & $1.40 \times 10^{-12}$ \\
\hline \multicolumn{7}{|c|}{$M_{X}=10^{3} \mathrm{GeV}$} \\
\hline
\end{tabular}

\begin{tabular}{|c|c|c|c|c|c|c|}
\hline \multirow[b]{3}{*}{ Parameters } & & & \multirow{3}{*}{$\begin{array}{c}\text { Perturbative } \\
\omega_{\phi}=0\end{array}$} & \multicolumn{3}{|c|}{ Constraints from reheating bound } \\
\hline & \multicolumn{2}{|c|}{ Case I (two-phase) } & & \multicolumn{2}{|c|}{ Case I (two-phase) } & \multirow{2}{*}{$\begin{array}{c}\text { Perturbative } \\
\omega_{\phi}=0\end{array}$} \\
\hline & $\omega_{\text {eff }}=10^{-3}$ & $\omega_{\text {eff }}=10^{-6}$ & & $\omega_{\text {eff }}=10^{-3}$ & $\omega_{\text {eff }}=10^{-6}$ & \\
\hline$n_{s}^{\min }$ & 0.96975 & 0.96975 & 0.9643 & 0.96975 & 0.96975 & 0.9651 \\
\hline$n_{s}^{\max }$ & 0.9719 & 0.9716 & 0.9722 & 0.9719 & 0.9716 & 0.9720 \\
\hline$\langle\sigma v\rangle_{\min }\left(\mathrm{GeV}^{-2}\right)$ & $3.80 \times 10^{-40}$ & $6.30 \times 10^{-37}$ & $9.10 \times 10^{-42}$ & $3.80 \times 10^{-40}$ & $6.30 \times 10^{-37}$ & $1.82 \times 10^{-41}$ \\
\hline$\langle\sigma v\rangle_{\max }\left(\mathrm{GeV}^{-2}\right)$ & $1.95 \times 10^{-33}$ & $7.32 \times 10^{-30}$ & $2.51 \times 10^{-5}$ & $1.95 \times 10^{-33}$ & $7.32 \times 10^{-30}$ & $7.42 \times 10^{-14}$ \\
\hline
\end{tabular}


constraints on the effective equation of state during the effective nonperturbative process calling it as phase I. However, during perturbative analysis due to explicit decay of the inflaton field into radiation, we obtain the nontrivial time-dependent effective equation of state. At this stage, let us remind the reader that in all the Planck analysis [109-111] on constraining the inflationary models $w_{\text {eff }}$ is assumed to be a constant free parameter during reheating, which follows from the proposal described in Ref. [11]. What we argue is that those assumptions should not be correct. After inflation, every inflationary model has its own characteristic oscillatory period, which contributes to the equation of state during reheating. Therefore, considering $w_{\text {eff }}$ as a free parameter loses some of the fundamental characteristic properties of the inflaton potential itself. Furthermore, if reheating occurs for a longer period of time, the timedependent $w_{\text {eff }}$ during the perturbative process should also be very important to get precise constraints on any inflationary model. This is where our analysis not only can play an important role in better understanding the inflationary models but also opens up the possibility of understanding the microphysics of the reheating process through $\mathrm{CMB}$ physics. As we can clearly see, the CMB power spectrum constrains the value of inflation-radiation coupling parametrized by $\Gamma_{\phi}$ through reheating temperature $T_{\text {re }}$. The usual connection between $\Gamma_{\phi}$ and $T_{\text {re }}$ will not be correct any more once we consider the decaying inflaton, as it is a well-known fact that during the reheating process, even at the end of reheating time, $\Gamma_{\phi}=H$, inflaton does not decay into radiation completely. Therefore, one certainly needs to take into account this fact while calculating $T_{\mathrm{re}}$ and its connection with the scalar power spectrum $n_{s}$ in the analysis. However, in all the previous theoretical as well as Planck analysis, complete decay of inflaton is assumed while relating the cosmological scales exiting and reentering the horizon at two different timescales. Therefore, based on the two-phase reheating scenario, our prediction of the reheating temperature corresponding to the inflationary power spectrum is more accurate than the previous analysis.

At first, we analyzed the viable constraints on the decay width as well as reheating parameters $\left(N_{\text {re }}, T_{\text {re }}\right)$ considering the decay of the inflaton field in the perturbative Boltzmann framework. Perturbative dynamics have been shown to give rise to a maximum reheating temperature $T_{\mathrm{re}}^{\max } \simeq 10^{15}$ naturally, which essentially corresponds to almost instantaneous reheating. As long as the decay width is in the perturbative regime, the result from the only perturbative process is trustworthy. However, because of the straightforward relation between $T_{\text {re }}$ and $\Gamma_{\phi}$, a high reheating temperature limit can correspond to nonperturbative phenomena. This fact motivates us to include nonperturbative aspects of reheating through effective dynamics. In our present scenario, the Universe passes through two distinct phases during reheating. Combining the inflation and subsequent standard big bang evolution with the intermediate two-phase reheating, our approach predicts the critical value of the inflaton decay constant $\Gamma_{\phi}^{\text {cri }}$ depending upon the phase-I equation of state $\omega_{\text {eff }}$.

The critical point naturally defined at $N_{\text {npre }}=N_{\text {pre }}$. Therefore, if $\Gamma_{\phi}<\Gamma_{\phi}^{\text {cri }}$, the reheating phase will be dominated by the perturbative one and vice versa. We also compare our numerical results of $\Gamma_{\phi}^{\mathrm{cri}}$ with the critical decay width obtained from the theoretical consideration for different types of inflaton-reheating field interactions $g \phi \chi^{2}, y \phi \chi^{3}$, and $h \phi \psi \bar{\psi}$. It turns out that all the theoretical values of $\Gamma_{\text {cri }}$ (model) correspond to an effective phase-I equation of state $\omega_{\text {eff }}$ within $10^{-3}-10^{-6}$. Our actual lattice simulation results also appeared to be compatible with this conclusion (see Fig. 3). A summary Table VII for $\Gamma_{\phi}^{\mathrm{cri}}$ is given for three observationally viable models.

The inclusion of the initial nonperturbative phase naturally changes the maximum reheating temperature value because of its perturbative definition. $T_{\mathrm{re}}^{\max }$ is no longer defined at the point of instantaneous reheating $N_{\text {re }} \simeq 0$; rather, it is defined at $N_{\text {re }} \approx N_{\text {npre }}$, which is equivalent to saying the phase-II $e$-folding number $N_{\text {pre }} \simeq 0$. At the end of phase I, approximately $50 \%$ of the total comoving energy density remains in the form of the inflaton, which naturally leads to different $T_{\mathrm{re}}^{\max }$ defined in the perturbative phase-II dynamics. This phase further sets the final equation of the state of the system to $1 / 3$. All these results have

TABLE VII. Different inflationary models and associated values of $\Gamma_{\phi}^{\mathrm{cri}}\left(T_{\mathrm{re}}^{\mathrm{cri}}\right)$, measured in units of GeV.

\begin{tabular}{|c|c|c|c|c|c|c|}
\hline & \multicolumn{2}{|c|}{$\alpha$-attractor } & \multicolumn{2}{|c|}{ Axion } & \multicolumn{2}{|c|}{ Minimal plateau } \\
\hline & $\alpha=1$ & $\alpha=100$ & $f=10 M_{p}$ & $f=50 M_{p}$ & $\phi_{*}=0.01 M_{p}$ & $\phi_{*}=0.001 M_{p}$ \\
\hline$T_{\mathrm{rr}}^{\mathrm{cri}}\left(\omega_{\mathrm{eff}}=10^{-3}\right)$ & $3.5 \times 10^{10}$ & $7.2 \times 10^{10}$ & $4.0 \times 10^{10}$ & $9.0 \times 10^{10}$ & $2.2 \times 10^{10}$ & $8.8 \times 10^{9}$ \\
\hline$T_{\mathrm{re}}^{\mathrm{cri}}\left(\omega_{\mathrm{eff}}=10^{-6}\right)$ & $2.3 \times 10^{5}$ & $1.2 \times 10^{6}$ & $1.8 \times 10^{5}$ & $1.9 \times 10^{5}$ & $2.8 \times 10^{5}$ & $5.8 \times 10^{4}$ \\
\hline$\Gamma_{\phi}^{\mathrm{cri}}\left(\omega_{\mathrm{eff}}=10^{-3}\right)$ & 960.0 & $1.3 \times 10^{4}$ & $3.7 \times 10^{4}$ & $2.7 \times 10^{4}$ & $2.3 \times 10^{3}$ & 394.7 \\
\hline$\Gamma_{\phi}^{\mathrm{cri}}\left(\omega_{\mathrm{eff}}=10^{-6}\right)$ & $1.4 \times 10^{-7}$ & $3.9 \times 10^{-6}$ & $1.1 \times 10^{-7}$ & $1.1 \times 10^{-7}$ & $4.8 \times 10^{-7}$ & $2.7 * 10^{-8}$ \\
\hline$\Gamma_{\phi}^{\mathrm{cri}}($ model $)(\phi \rightarrow \chi \chi \chi)$ & 0.07 & 0.01 & $2.8 \times 10^{-3}$ & $3.2 \times 10^{-3}$ & 0.70 & 15.30 \\
\hline$\Gamma_{\phi}^{\mathrm{cri}}($ model $)(\phi \rightarrow \chi \chi)$ & 5.03 & 1.60 & 0.45 & 0.51 & 34.20 & 231.70 \\
\hline$\Gamma_{\phi}^{\mathrm{cri}}(\operatorname{model})(\phi \rightarrow \bar{\psi} \psi)$ & 260.30 & 42.20 & 10.80 & 12.30 & $2.7 \times 10^{3}$ & $5.8 \times 10^{4}$ \\
\hline
\end{tabular}


TABLE VIII. Models and their associated values of $M_{X}^{\max }$, measured in units of GeV.

\begin{tabular}{|c|c|c|c|c|c|c|}
\hline \multicolumn{7}{|c|}{ Higgs-Starobinsky model } \\
\hline & \multicolumn{2}{|c|}{$\gamma=10^{-12}$} & \multicolumn{2}{|c|}{$\gamma=10^{-8}$} & \multicolumn{2}{|c|}{$\gamma=10^{-4}$} \\
\hline & $\omega_{\text {eff }}=10^{-3}$ & $\omega_{\text {eff }}=10^{-6}$ & $\omega_{\mathrm{eff}}=10^{-3}$ & $\omega_{\text {eff }}=10^{-6}$ & $\omega_{\text {eff }}=10^{-3}$ & $\omega_{\text {eff }}=10^{-6}$ \\
\hline$M_{X}^{\max }(\operatorname{minimum})$ & $1.4 \times 10^{4}$ & $1.1 \times 10^{5}$ & 1.4 & 10.6 & $1.4 \times 10^{-4}$ & $1.1 \times 10^{-3}$ \\
\hline$M_{X}^{\max }(\operatorname{maximum})$ & $11.7 \times 10^{8}$ & $11.6 \times 10^{8}$ & $11.7 \times 10^{4}$ & $11.6 \times 10^{4}$ & 11.7 & 11.6 \\
\hline \multicolumn{7}{|c|}{ Minimal plateau model $\left(\phi_{*}=0.001 M_{p}\right)$} \\
\hline & \multicolumn{2}{|c|}{$\gamma=10^{-12}$} & \multicolumn{2}{|c|}{$\gamma=10^{-8}$} & \multicolumn{2}{|c|}{$\gamma=10^{-4}$} \\
\hline & $\omega_{\mathrm{eff}}=10^{-3}$ & $\omega_{\text {eff }}=10^{-6}$ & $\omega_{\text {eff }}=10^{-3}$ & $\omega_{\text {eff }}=10^{-6}$ & $\omega_{\text {eff }}=10^{-3}$ & $\omega_{\text {eff }}=10^{-6}$ \\
\hline$M_{X}^{\max }(\operatorname{minimum})$ & $1.4 \times 10^{4}$ & $1.0 \times 10^{5}$ & 1.4 & 10.4 & $1.4 \times 10^{-4}$ & $1.0 \times 10^{-3}$ \\
\hline$M_{X}^{\max }(\operatorname{maximum})$ & $6.8 \times 10^{8}$ & $6.7 \times 10^{8}$ & $6.8 \times 10^{4}$ & $6.7 \times 10^{4}$ & 6.8 & 6.7 \\
\hline
\end{tabular}

been shown to be crucially dependent upon the phase-I effective equation of state $\omega_{\text {eff }}$. As one changes the value of $\omega_{\text {eff }}$ from $10^{-3} \rightarrow 10^{-6}$, the phase-I $e$-folding number $N_{\text {npre }}$ changes from $6 \rightarrow 12$. The maximum reheating temperature $T_{\mathrm{re}}^{\max }$ accordingly changes from $\left(10^{13} \rightarrow 10^{10}\right) \mathrm{GeV}$. Therefore, the conclusion that can be emphasized is that the value of the reheating temperature may encode the information about the nonperturbative phase. Furthermore, all the inflationary models which are compatible with the observed CMB anisotropy predict the same maximum reheating temperature $\left(T_{\mathrm{re}}^{\max }\right)$ for a given $\omega_{\text {eff }}$. This is reminiscent of the maximum reheating temperature $T_{\mathrm{re}}^{\max }$ obtained for purely perturbative reheating dynamics irrespective of the inflation model. Keeping this point in mind, we have performed a comparative analysis of different existing reheating formalisms such as conventional reheating dynamics (case II) and purely perturbative analysis (case III) with our proposed two-phase (case I). For both cases II and III, the model-independent maximum value of the reheating temperature turns out to be $T_{\mathrm{re}}^{\max } \simeq 10^{15} \mathrm{GeV}$, which is reduced to $10^{13}-10^{10} \mathrm{GeV}$ when considering two-phase reheating for $\omega_{\text {eff }}=10^{-3}-10^{-6}$. Furthermore, the two-phase reheating scenario constrains the inflation model within a very narrow range of allowed scalar spectral index compatible with CMB anisotropy.

Further generalization has been analyzed by including the dark matter component as one of the decay products of the inflaton. Depending upon the mass dark matter annihilation cross section versus scalar spectral index parameter space has been shown to be reduced because of two-phase reheating as compared to that of standard reheating dynamics, which can be observed from Figs. 9 and 10 . Details of the allowed parameter space for various models can be obtained from Tables V and VI. Because of the nontrivial initial condition for two-phase dynamics, there exists a maximum possible mass $M_{X}^{\max }$ above which dark matter turned out to be overproduced no matter how small the annihilation cross section is assumed. In the summary
Table VIII, we provide numerical values of maximum possible dark matter mass allowed for different viable models under consideration. As just stated, the value of $M_{X}^{\max }$ is directly connected to $\gamma\left(\gamma=\frac{\rho_{X}}{\rho_{R}}\right)$, which is defined during phase I. Once we fixed the spectral index for a particular inflation model, these values of $M_{X}^{\max }$ are nearly independent of the choice of $\omega_{\text {eff }}$.

Nonetheless, one important point we should understand that the existing reheating scenarios, either perturbative or nonperturbative, are not the complete description of this phase. A unified description that connects both nonperturbative and perturbative dynamics is more appropriate. In our present study, we, for the first time, try to construct such a unified description. As a first attempt toward this goal, we describe nonperturbative preheating dynamics by effective dynamics. Our present formalism is particularly suited for the class of inflation models with a quadratic potential near its minimum. For an inflaton potential with a power greater than 2, lattice results generically predict the equation of state $\frac{1}{3}$ after the end of nonperturbative dynamics [37]. So for those models, our two-phase reheating is not applicable. We will be considering this case in our future work. Instead of considering an effective nonperturbative approach, actual nonperturbative dynamics integrated with perturbative one would be more appropriate. Recently, an interesting approach has been proposed to describe preheating phenomena in the Boltzmann framework [112]. In our present two-phase reheating dynamics, the aforementioned nonperturbative Boltzmann framework could be natural to integrate with the perturbative Boltzmann equations. Another important fact we have not considered is the temperature dependency of the effective numbers of relativistic degrees of freedom $\left(g_{*}\right)$. Constant effective degrees of freedom is a reasonably good approximation for a wide range of temperature $[113,114]$ till the QCD hadronic transition happens at around $10^{2} \mathrm{MeV}$ scale, around which the value of effective degrees of freedom changes as $g_{*}=$ $100 \rightarrow 10[115,116]$. So our eventual plan in the future is to 
calculate dark matter and reheating parameter space accurately by acknowledging the precise evolution of those degrees of freedom in the thermal bath [117-119].

\section{ACKNOWLEDGMENTS}

We thank the HEP and Gravity groups at IIT Guwahati for useful discussions. P. S. thanks the cluster computing facility at IIT Madras, where part of the numerical simulations were carried out. The research of P. S. is partly supported through the Core Research Grant No. CRG/ 2018/002200 from Science and Engineering Research Board, Department of Science and Technology, Government of India.

\section{APPENDIX A: TWO-PHASE REHEATING: ANALYTIC EXPRESSION OF $\boldsymbol{T}_{\max }$}

After the end of the effective nonperturbative dynamics, the usual perturbative analysis follows, and the governing Boltzmann equations are

$$
\dot{\rho_{\phi}}+3 H\left(1+\omega_{\phi}^{1}\right) \rho_{\phi}=-\Gamma_{\phi} \rho_{\phi}\left(1+\omega_{\phi}^{1}\right)
$$

$$
\dot{\rho}_{R}+4 H \rho_{R}=\Gamma_{\phi} \rho_{\phi}\left(1+\omega_{\phi}^{1}\right)+2\left\langle E_{X}\right\rangle\langle\sigma v\rangle\left(n_{X}^{2}-n_{X, \mathrm{eq}}^{2}\right),
$$

$$
\dot{n_{X}}+3 H n_{X}=-\langle\sigma v\rangle\left(n_{X}^{2}-n_{X, \mathrm{eq}}^{2}\right) .
$$

In order to solve analytically, we assume the inflaton energy density to follow the equation

$\rho_{\phi}=\rho_{\phi}^{\text {in }}\left(\frac{a}{a_{\text {in }}}\right)^{-3\left(1+\omega_{\phi}^{1}\right)} e^{-\Gamma_{\phi}\left(1+\omega_{\phi}^{1}\right)\left(t-t_{i}\right)} \simeq \rho_{\phi}^{\text {in }}\left(\frac{a}{a_{\text {in }}}\right)^{-3\left(1+\omega_{\phi}^{1}\right)}$.

Here $\Gamma_{\phi}$ is the time-independent inflaton decay constant. Notice that the effect of the decay constant is being ignored assuming the fact that at the initial stage of perturbative reheating inflaton energy is the dominant one. $\rho_{\phi}^{i}$ and $t_{i}$ are initial density and initial time during the perturbative era, respectively. Using the above equation, the radiation energy can be solved as follows:

$$
\begin{aligned}
d\left(\rho_{R} a^{4}\right) & =\left(\Gamma_{\phi} \rho_{\phi}\left(1+\omega_{\phi}^{1}\right) a^{4}+2\left\langle E_{X}\right\rangle\langle\sigma v\rangle\left(n_{X}^{2}-n_{X, \mathrm{eq}}^{2}\right) a^{4}\right) d t \\
& =\left(\Gamma_{\phi} \rho_{\phi}^{\mathrm{in}} e^{-\Gamma_{\phi}\left(t-t_{i}\right)} a_{\mathrm{in}}^{3\left(1+\omega_{\phi}^{1}\right)} a^{1-3 \omega_{\phi}^{1}}\left(1+\omega_{\phi}^{1}\right)+2\left\langle E_{X}\right\rangle\langle\sigma v\rangle\left(n_{X}^{2}-n_{X, \mathrm{eq}}^{2}\right) a^{4}\right) d t \\
& \simeq \Gamma_{\phi} \rho_{\phi}^{\mathrm{in}} a_{\mathrm{in}}^{3\left(1+\omega_{\phi}^{1}\right)} a^{-3 \omega_{\phi}^{1}} \frac{d a}{H}+2\left\langle E_{X}\right\rangle\langle\sigma v\rangle\left(n_{X}^{2}-n_{X, \mathrm{eq}}^{2}\right) a^{3} \frac{d a}{H} .
\end{aligned}
$$

We use the following expression for the Hubble parameter:

$$
H=\frac{\sqrt{\rho_{\phi}^{\mathrm{in}}\left(\frac{a}{a_{\mathrm{in}}}\right)^{-3\left(1+\omega_{\phi}^{\mathrm{1}}\right)}+\rho_{R}^{\mathrm{in}}\left(\frac{a}{a_{\mathrm{in}}}\right)^{-4}}}{\sqrt{3} M_{p}},
$$

where $\rho_{R}^{\text {in }}$ is the initial radiation density at the beginning of the perturbative phase. For the reheating temperature computation, we ignore the effect of dark matter whose contribution has been verified to be negligible in our full numerical computation. By solving Eq. (A5), we obtain

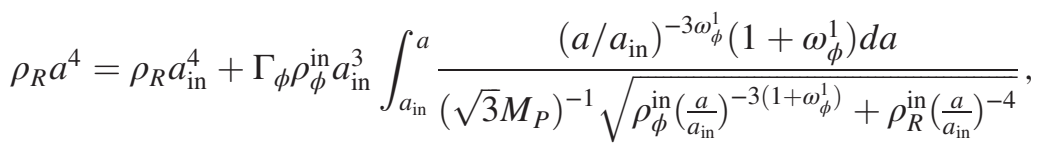

$$
\begin{aligned}
& \rho_{R} x^{4}=\rho_{R}^{\text {in }}+\Gamma_{\phi} \rho_{\phi}^{\text {in }}\left(1+\omega_{\phi}^{1}\right) \int_{1}^{x} \frac{x^{2-3 \omega_{\phi}^{1}} d x}{\left(\sqrt{3} M_{P}\right)^{-1} \sqrt{\rho_{\phi}^{\text {in }} x^{1-3 \omega_{\phi}^{1}}+\rho_{R}^{\text {in }}}} \\
& =\rho_{R}^{\text {in }}+\frac{\Gamma_{\phi} \rho_{\phi}^{\text {in }}\left(1+\omega_{\phi}^{1}\right)}{H_{\text {in }}} \int_{1}^{x} \frac{x^{(3-c) / 2} d x}{\sqrt{1+\frac{\rho_{R}^{\text {in }}}{\rho_{\phi}^{\text {in }}} x^{c-1}}} \simeq \rho_{R}^{\text {in }}+\frac{\Gamma_{\phi} \rho_{\phi}^{\text {in }}\left(1+\omega_{\phi}^{1}\right)}{H_{\text {in }}} \int_{1}^{x} x^{(3-c) / 2}\left(1-\frac{\rho_{R}^{\text {in }}}{2 \rho_{\phi}^{\text {in }}} x^{c-1}\right) d x \text {. }
\end{aligned}
$$

In the above expression, we neglected higher-order terms of $\rho_{R}^{\text {in }} / \rho_{\phi}^{\text {in }}$. Additionally, in terms of radiation temperature $T_{\text {rad }}=\left(\frac{30}{\pi^{2} g_{*}} \rho_{R}\right)^{1 / 4}$, the above equation transforms into the following expression: 


$$
\frac{\beta T^{4} x^{4}}{\rho_{\phi}^{\text {in }}}=\frac{\Gamma_{\phi}\left(1+\omega_{\phi}^{1}\right)}{H_{\text {in }}}\left[\frac{2}{5-c}\left(x^{(5-c) / 2}-1\right)+\frac{\rho_{R}^{\text {in }}}{\rho_{\phi}^{\text {in }}}\left(\frac{1-x^{(c+3) / 2}}{c+3}+\frac{H_{\text {in }}}{\Gamma_{\phi}\left(1+\omega_{\phi}^{1}\right)}\right)\right] .
$$

Here $x, \beta, c$, and $H_{\text {in }}$ are defined, respectively, as

$$
x=\frac{a}{a_{\text {in }}}, \quad \beta=\frac{\pi^{2} g_{*}(T)}{30}, \quad c=3 \omega_{\phi}^{1}, \quad H_{\text {in }}=\frac{\sqrt{\rho_{\phi}^{\text {in }}}}{\sqrt{3} M_{P}} .
$$

The maximum radiation temperature can be found by taking derivative of the above equation (A8) with respect to $x$ and setting it to zero:

$$
\frac{4 \beta T^{3}}{\rho_{\phi}^{\text {in }}} \frac{d T}{d x}=\frac{-4 \Gamma_{\phi}\left(1+\omega_{\phi}^{1}\right)}{H_{\text {in }} x^{5}}\left[\frac{\frac{3+c}{4} x^{(5-c) / 2}-2}{5-c}+\frac{\rho_{R}^{\text {in }}}{\rho_{\phi}^{\text {in }}}\left(\frac{\frac{c-5}{8} x^{(c+3) / 2}+1}{c+3}+\frac{H_{\text {in }}}{\Gamma_{\phi}\left(1+\omega_{\phi}^{1}\right)}\right)\right]=0 .
$$

In the limit of $\rho_{\phi} / \rho_{R} \ll 1$ (perturbative approximation), the values of $x$ at the point of maximum radiation temperature appear as

$$
x_{\max , p}=\left(\frac{8}{3+c}\right)^{2 /(5-c)}
$$

In our present analysis, the expression of $x$ associated with the maximum radiation temperature leads to the following relation:

$$
x_{\max } \simeq\left(\frac{8}{3+c}\right)^{2 /(5-c)}\left[1-\frac{\rho_{R}^{\mathrm{in}}}{\rho_{\phi}^{\mathrm{in}}}\left(\frac{c-5}{8(c+3)} x_{\max , p}^{(c+3) / 2}+\frac{1}{c+3}+\frac{H_{\mathrm{in}}}{\Gamma_{\phi}\left(1+\omega_{\phi}^{1}\right)}\right)\right]=x_{\max , p}[1-z],
$$

where $z=\frac{\rho_{R}^{\text {in }}}{\rho_{\phi}^{\text {in }}}\left(\frac{c-5}{8(c+3)} x_{\max , p}^{(c+3) / 2}+\frac{1}{c+3}+\frac{H_{\text {in }}}{\Gamma_{\phi}\left(1+\omega_{\phi}^{1}\right)}\right)$. Now after replacing the expression of $x_{\max }$ into the above Eq. (A8), the maximum radiation temperature turns out as

$$
\begin{aligned}
T_{\max } & \simeq\left(\frac{\Gamma_{\phi}\left(1+\omega_{\phi}^{1}\right) \rho_{\phi}^{\mathrm{in}}}{\beta H_{\mathrm{in}} x_{\max , p}^{4}} \frac{2}{3+c}\right)^{1 / 4}\left[1+\frac{3+c}{2} \frac{\rho_{R}^{\text {in }}}{\rho_{\phi}^{\text {in }}}\left(\frac{1-x_{\max , p}^{(c+3) / 2}}{c+3}+\frac{H_{\mathrm{in}}}{\Gamma_{\phi}\left(1+\omega_{\phi}^{1}\right)}\right)\right]^{1 / 4} \\
& \simeq\left(\frac{\Gamma_{\phi}\left(1+\omega_{\phi}^{1}\right) \rho_{\phi}^{\text {in }}}{\beta H_{\text {in }} x_{\max , p}^{4}} \frac{2}{3+c}\right)^{1 / 4}\left[1+\frac{3+c}{8} \frac{\rho_{R}^{\text {in }}}{\rho_{\phi}^{\text {in }}}\left(\frac{1-x_{\max , p}^{(c+3) / 2}}{c+3}+\frac{H_{\text {in }}}{\Gamma_{\phi}\left(1+\omega_{\phi}^{1}\right)}\right)\right] .
\end{aligned}
$$

In the above expression, we have neglected higher-order terms of $\rho_{R}^{\text {in }} / \rho_{\phi}^{\text {in }}$. Next, we will try to express all initial densities in terms of the inflaton energy density at the end of the inflation $\rho_{\phi}^{\text {end }}$. The effective nonperturbative phase-I dynamics solves the radiation and inflaton energy density in terms of $\rho_{\phi}^{\text {end }}$. Therefore, during phase $\mathrm{I}$, the dimensionless radiation energy density $R^{I}(A)$ can be correlated with inflaton energy density $\Phi^{I}(A)$ [using (36), (37), and (41)] as

$$
R^{I}(A)=\frac{3 \omega_{\mathrm{eff}}}{\left(1-3 \omega_{\mathrm{eff}}\right)} \Phi^{I}(A) A
$$

The initial densities during phase II (perturbative era) in terms of dimensionless comoving energy densities are identified as

$$
\rho_{\phi}^{\text {in }}=\Phi\left(A_{\text {npre }}\right) A_{\text {npre }}^{-3\left(1+\omega_{\phi}^{1}\right)} m_{\phi}^{4}, \quad \rho_{R}^{\text {in }}=R\left(A_{\text {npre }}\right) A_{\text {npre }}^{-4} m_{\phi}^{4} .
$$

Furthermore, we can relate the $\Phi\left(A_{\text {npre }}\right)$ in terms of $\Phi(A=1)$ as

$$
\Phi\left(A_{\text {npre }}\right)=\left(1-3 \omega_{\text {eff }}\right) \Phi(A=1) A_{\text {npre }}^{-3 \omega_{\text {eff }}}
$$


where $A_{\text {npre }}$ is the normalized scale factor at the end of the effective dynamics. $A_{\text {npre }}$ is defined when the dimensionless comoving radiation energy density becomes $50 \%$ of the total comoving energy density, $\frac{R\left(A_{\text {npre }}\right)}{\Phi\left(A_{\text {npre }}\right)+R\left(A_{\text {npre }}\right)} \simeq \frac{1}{2} \Rightarrow$ $\Phi\left(A_{\text {npre }}\right) \simeq R\left(A_{\text {npre }}\right)$. Using Eq. (A15), one can find $A_{\text {npre }}$ and corresponding $e$-folding number $N_{\text {npre }}$, respectively, as

$$
A_{\text {npre }}=\frac{1-3 \omega_{\text {eff }}}{3 \omega_{\text {eff }}}, \quad N_{\text {npre }}=\ln \left(A_{\text {npre }}\right) .
$$

From our analytic expression above, we obtain $N_{\text {npre }} \sim(5.8,12.7)$ for two values of $\omega_{\text {eff }}=\left(10^{-3}, 10^{-6}\right)$ accordingly. These values of the $e$-folding number during phase I almost exactly match with our numerical result.

The final expression for the maximum radiation temperature in terms of comoving energy densities is given by

$$
T_{\max } \simeq D^{1 / 4}\left[1+\frac{(3+c) R\left(A_{\text {npre }}\right)}{8 \Phi\left(A_{\text {npre }}\right) A_{\text {npre }}^{1-c}}\left(\frac{1-x_{\text {max }, p}^{(c+3) / 2}}{c+3}+\frac{\sqrt{\Phi\left(A_{\text {npre }}\right) A_{\text {npre }}^{-3\left(1+\omega_{\phi}^{1}\right)} m_{\phi}^{4}}}{\sqrt{3} M_{p} \Gamma_{\phi}\left(1+\omega_{\phi}^{1}\right)}\right)\right],
$$

where

$$
D=\left(\frac{2 \Gamma_{\phi}\left(1+\omega_{\phi}^{1}\right) \sqrt{3 M_{p}^{2} \Phi\left(A_{\text {npre }}\right) A_{\text {npre }}^{-3\left(1+\omega_{\phi}^{1}\right)} m_{\phi}^{4}}}{(3+c) \beta x_{\max , p}^{4}}\right)^{1 / 4} .
$$

Combining equations from (A15) to (A20), we obtain the maximum radiation temperature as a function of $\Phi(A=1)$ (dimensionless comoving inflaton energy density at the end of the inflation).

\section{APPENDIX B: TWO PHASE REHEATING: ANALYTIC EXPRESSION OF INFLATON DECAY WIDTH $\Gamma_{\phi}$ AND $T_{\text {re }}$}

Assuming the end point of reheating as $x_{\mathrm{re}}=a_{\mathrm{re}} / a_{\text {npre }}$ and considering Eq. (A8), the reheating temperature can be obtained as

$$
T_{\mathrm{re}}^{4}=\frac{\Gamma_{\phi} \rho_{\phi}^{\mathrm{in}}\left(1+\omega_{\phi}^{1}\right) x_{\mathrm{re}}^{-4}}{\beta H_{\mathrm{in}}}\left[\frac{2}{5-c}\left(x_{\mathrm{re}}^{(5-c) / 2}-1\right)+\frac{\rho_{R}^{\text {in }}}{\rho_{\phi}^{\text {in }}}\left(\frac{1-x_{\mathrm{re}}^{(c+3) / 2}}{c+3}+\frac{H_{\mathrm{in}}}{\Gamma_{\phi}\left(1+\omega_{\phi}^{1}\right)}\right)\right] .
$$

Using Eq. (55) (entropy conservation of thermal radiation), one arrives at the following relation:

$$
T_{\mathrm{re}}^{4}=\left(\frac{43}{11 g_{\mathrm{re}}}\right)^{4 / 3}\left(\frac{a_{0} T_{0}}{k}\right)^{4} H_{k}^{4} e^{-4 N_{k}} e^{-4 N_{\mathrm{npre}}} e^{-4 N_{\mathrm{pre}}}=G^{4}\left(\frac{a_{\mathrm{re}}}{a_{\mathrm{npre}}}\right)^{-4}=G^{4} x_{\mathrm{re}}^{-4}
$$

where

$$
G=\left(\frac{43}{11 g_{\mathrm{re}}}\right)^{1 / 3}\left(\frac{a_{0} T_{0}}{k}\right) H_{k} e^{-N_{k}} e^{-N_{\text {npre }}} .
$$

Comparing Eqs. (B1) and (B2), we obtain $\Gamma_{\phi}$ in terms of $x_{\mathrm{re}}$ :

$$
\begin{aligned}
\Gamma_{\phi} & =\left(\frac{G^{4} \beta}{\rho_{\phi}^{\text {in }}}-\frac{\rho_{R}^{\text {in }}}{\rho_{\phi}^{\text {in }}}\right) \frac{H_{\mathrm{in}}}{\left(1+\omega_{\phi}^{1}\right)}\left[\frac{2}{5-c}\left(x_{\mathrm{re}}^{(5-c) / 2}-1\right)+\frac{\rho_{R}^{\mathrm{in}}}{\rho_{\phi}^{\text {in }}}\left(\frac{1-x_{\mathrm{re}}^{(c+3) / 2}}{c+3}\right)\right]^{-1} \\
& \simeq\left(\frac{G^{4} \beta}{\rho_{\phi}^{\text {in }}}-\frac{\rho_{R}^{\text {in }}}{\rho_{\phi}^{\text {in }}}\right) \frac{H_{\mathrm{in}}}{\left(1+\omega_{\phi}^{1}\right)} \frac{5-c}{2} x_{\mathrm{re}}^{(c-5) / 2}\left[1+\frac{5-c}{2(c+3)} \frac{\rho_{R}^{\text {in }}}{\rho_{\phi}^{\text {in }}} x_{\mathrm{re}}^{c-1}\right] .
\end{aligned}
$$

The reheating temperature is defined when the inflaton field comes in thermal equilibrium with the radiation bath at the point 


$$
H\left(x_{\mathrm{re}}\right)^{2}=\frac{\rho_{\phi}\left(x_{\mathrm{re}}\right)+\rho_{R}\left(x_{\mathrm{re}}\right)}{3 M_{p}^{2}} \simeq \frac{\rho_{R}\left(x_{\mathrm{re}}\right)}{3 M_{p}^{2}}=\Gamma_{\phi}^{2}
$$

In the above equation, we ignore the contribution of inflaton energy density to be negligible. Using the expression for the radiation energy density, we can obtain the decay width as follows:

$$
\Gamma_{\phi}^{2} \simeq\left(\frac{G^{4} \beta}{\rho_{\phi}^{\mathrm{in}}}-\frac{\rho_{R}^{\mathrm{in}}}{\rho_{\phi}^{\mathrm{in}}}\right)^{2} \frac{H_{\mathrm{in}}^{2}}{\left(1+\omega_{\phi}^{1}\right)^{2}}\left(\frac{5-c}{2}\right)^{2} x_{\mathrm{re}}^{c-5}\left[1+\frac{5-c}{c+3} \frac{\rho_{R}^{\mathrm{in}}}{\rho_{\phi}^{\mathrm{in}}} x_{\mathrm{re}}^{c-1}\right] .
$$

In the earlier expression, we can ignore the second term in the third bracket, since $x_{\mathrm{re}} \gg 1$ for most of the values of the spectral index. As a result, the $\Gamma_{\phi}^{2}$ can now be written as

$$
\Gamma_{\phi}^{2} \simeq\left(\frac{G^{4} \beta}{\rho_{\phi}^{\text {in }}}-\frac{\rho_{R}^{\text {in }}}{\rho_{\phi}^{\text {in }}}\right)^{2} \frac{H_{\mathrm{in}}^{2}}{\left(1+\omega_{\phi}^{1}\right)^{2}}\left(\frac{5-c}{2}\right)^{2} x_{\mathrm{re}}^{c-5} .
$$

Furthermore, the radiation energy density at the ending point of reheating era $\rho_{R}\left(x_{\mathrm{re}}\right)$ can be expressed as

$$
\rho_{R}\left(x_{\mathrm{re}}\right) \simeq x_{\mathrm{re}}^{-4} \rho_{\phi}^{\text {in }}\left[\frac{2}{5-c} \frac{\Gamma_{\phi}\left(1+\omega_{\phi}^{1}\right)}{H_{\mathrm{in}}} x_{\mathrm{re}}^{(5-c) / 2}+\frac{\rho_{R}^{\text {in }}}{\rho_{\phi}^{\text {in }}}\left(1-\frac{\Gamma_{\phi}\left(1+\omega_{\phi}^{1}\right)}{H_{\mathrm{in}}} \frac{x_{\mathrm{re}}^{(c+3) / 2}}{c+3}\right)\right] .
$$

Combining Eqs. (B4) and (B8), one can find

$$
\rho_{R}\left(x_{\mathrm{re}}\right)=\beta T_{\mathrm{re}}^{4} \simeq x_{\mathrm{re}}^{-4} \rho_{\phi}^{\text {in }}\left[\frac{G^{4} \beta}{\rho_{\phi}^{\text {in }}}+\frac{5-c}{2(c+3)} \frac{\rho_{R}^{\text {in }}}{\rho_{\phi}^{\text {in }}}\left(\frac{G^{4} \beta}{\rho_{\phi}^{\text {in }}}-\frac{\rho_{R}^{\text {in }}}{\rho_{\phi}^{\text {in }}}\right) x_{\mathrm{re}}^{c-1}\right] .
$$

Now equating the above equation with $\Gamma_{\phi}^{2}$ [Eq. (B7)], one arrives at the following expression:

$$
x_{\mathrm{re}}=\left(\frac{\alpha}{\eta}\right)^{1 /(c-1)}
$$

Here

$$
\alpha=\frac{G^{4} \beta}{\rho_{\phi}^{\text {in }}}, \quad \eta=\frac{5-c}{2}\left(\frac{G^{4} \beta}{\rho_{\phi}^{\text {in }}}-\frac{\rho_{R}^{\text {in }}}{\rho_{\phi}^{\text {in }}}\right)\left[\frac{\rho_{R}^{\text {in }}}{(c+3) \rho_{\phi}^{\text {in }}}+\frac{5-c}{2} \frac{3 M_{p}^{2} H_{\text {in }}^{2}}{\rho_{\phi}^{\text {in }}\left(1+\omega_{\phi}^{1}\right)^{2}}\left(\frac{G^{4} \beta}{\rho_{\phi}^{\text {in }}}-\frac{\rho_{R}^{\text {in }}}{\rho_{\phi}^{\text {in }}}\right)\right] .
$$
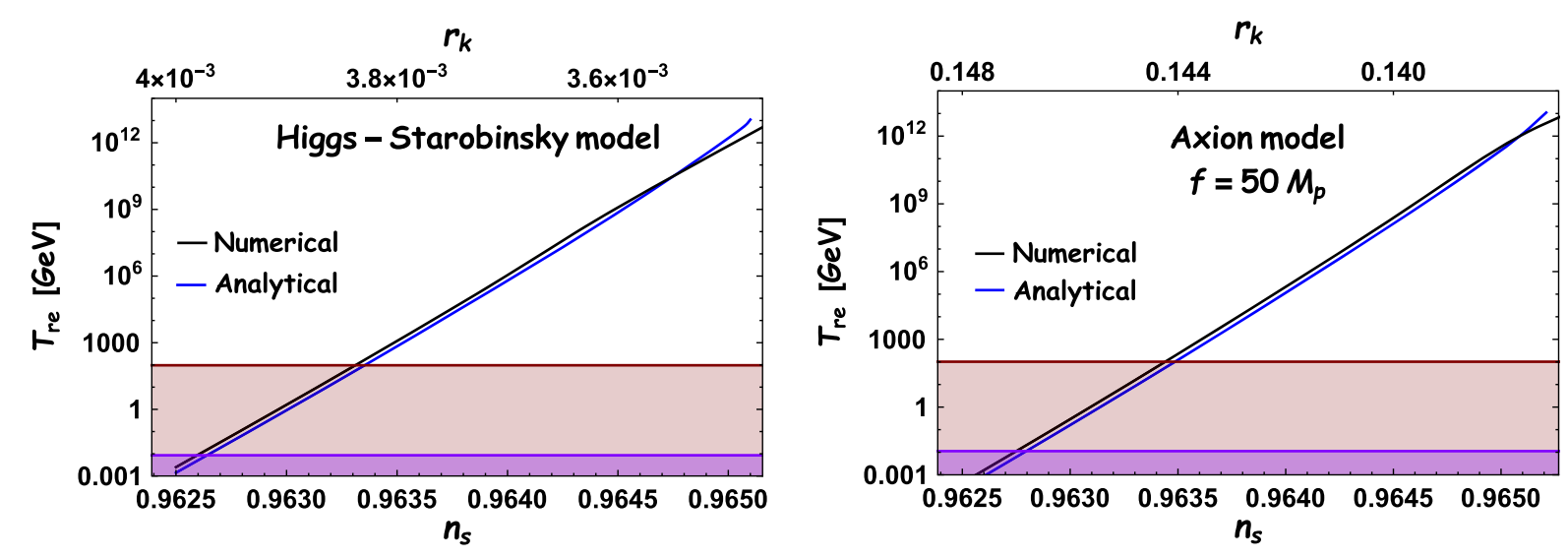

FIG. 11. Variation of reheating temperature $\left(T_{\text {re }}\right)$ as a function of $n_{s}$ for the Higgs-Starobinsky and axion inflation models with $\omega_{\text {eff }}=10^{-3}$ in the framework of two-phase analysis. The solid blue line indicates the result from approximate analytical expression [Eq. (B1)], whereas the solid black line shows results from numerical analysis. The light brown region is below the electroweak scale $T_{\text {ew }} \backsim 100 \mathrm{GeV}$, and the violet region below $10^{-2} \mathrm{GeV}$ would ruin the predictions of BBN. 
By utilizing the above equation, we can easily fix the decay width [Eq. (B4)] and reheating temperature [Eq. (B9)] as they are the functions of $x_{\mathrm{re}}$. Besides, the maximum reheating temperature and associated maximum possible value of the spectral index $\left(n_{s}^{\max }\right)$ can also be defined at the point $x_{\text {re }} \rightarrow 1\left(N_{\text {pre }} \rightarrow 0\right)$. To check whether our analytical calculations predict the correct result, we plot the reheating temperature as a function of the spectral index (Fig. 11) and compare with our numerical result.

\section{APPENDIX C: TWO-PHASE REHEATING: ANALYTICAL EXPRESSION OF DARK MATTER ABUNDANCE AND ORIGIN OF MAXIMUM DARK MATTER MASS $M_{X}^{\max }$}

The relevant Boltzmann equation for the evolution of dark matter during the perturbative reheating phase is expressed as

$d\left(n_{X} a^{3}\right)=-a^{3}\langle\sigma v\rangle\left[n_{X}^{2}-n_{X, \mathrm{eq}}^{2}\right] d t=-\frac{a^{3}\langle\sigma v\rangle\left[n_{X}^{2}-n_{X, \mathrm{eq}}^{2}\right] d a}{a H}$.

Throughout our calculation, we assume dark matter particles are always relativistic and never attain chemical equilibrium $\left(n_{X} \ll n_{X, \text { eq }}\right)$ with the radiation bath. Hence, in this freeze-in scenario, the dark matter density always remains subdominant compared to its thermal equilibrium values. Consequently, the above dark matter evolution equation can be approximated as

$$
d\left(n_{X} a^{3}\right)=\frac{a^{3}\langle\sigma v\rangle n_{X, \mathrm{eq}}^{2}}{a H} d a
$$

In the relativistic limit, the equilibrium distribution is given by

$$
n_{X, \mathrm{eq}}=\frac{g T^{3}}{\pi^{2}}
$$

where $g$ is the number of degrees of freedom of the dark matter species. Furthermore, considering the relativistic dark matter, the dark matter's mass must be less than the reheating temperature. So the freeze-in happens very late after the reheating. Therefore, we can approximate the Hubble parameter as

$$
H(a)=\sqrt{\frac{\rho_{\phi}(a)+\rho_{R}(a)}{3 M_{p}^{2}}} \simeq \sqrt{\frac{\rho_{R}}{3 M_{p}^{2}}} .
$$

Connecting Eqs. (C2)-(C4), one can solve for the dark matter component as

$$
\begin{aligned}
& n_{X}^{f} a_{f}^{3}=n_{X}^{\text {in }} a_{\mathrm{in}}^{3}+\int_{a_{\mathrm{in}}}^{a_{f}} \frac{a^{2}\langle\sigma v\rangle \frac{g^{2}}{\pi^{4}} \beta^{-3 / 2} \rho_{R}}{\left(\sqrt{3} M_{P}\right)^{-1}} d a, \\
& n_{X}^{f} x_{f}^{3} \simeq n_{X}^{\text {in }}+\int_{1}^{x_{f}} \frac{\langle\sigma v\rangle g^{2} \sqrt{3} M_{P}}{\pi^{4} \beta^{3 / 2}} \rho_{\phi}^{\text {in }}\left[\frac{2 \Gamma_{\phi}\left(1+\omega_{\phi}^{1}\right)}{H_{\mathrm{in}}(5-c)} x^{(1-c) / 2}+\frac{\rho_{R}^{\text {in }}}{\rho_{\phi}^{\text {in }}}\left(x^{-2}-\frac{\Gamma_{\phi}\left(1+\omega_{\phi}^{1}\right)}{H_{\mathrm{in}}(c+3)} x^{(c-1) / 2}\right)\right] d a,
\end{aligned}
$$

where $x_{f}=\frac{a_{f}}{a_{\text {in }}}$ and

$$
\rho_{R} \simeq \rho_{\phi}^{\mathrm{in}} x^{-4}\left[\frac{2 \Gamma_{\phi}\left(1+\omega_{\phi}^{1}\right)}{H_{\mathrm{in}}(5-c)} x^{(5-c) / 2}+\frac{\rho_{R}^{\mathrm{in}}}{\rho_{\phi}^{\mathrm{in}}}\left(1-\frac{\Gamma_{\phi}\left(1+\omega_{\phi}^{1}\right)}{H_{\mathrm{in}}(c+3)} x^{(c+3) / 2}\right)\right] .
$$

The scale factor at the point of freeze-in is defined as $a_{f}$, when both comoving dark matter and radiation component become constant. In the preceding expression, we ignore higher-order terms of $\rho_{R}^{\text {in }} / \rho_{\phi}^{\text {in }}$. With these assumptions the comoving number density $n_{X}^{f}$ is found to be

$$
n_{X}^{f} x_{f}^{3} \simeq n_{X}^{\text {in }}+\langle\sigma v\rangle f\left(x_{f}\right),
$$

where $f\left(x_{f}\right)$ can be expressed as

$$
f\left(x_{f}\right) \simeq \rho_{\phi}^{\text {in }}\left[\frac{4 \Gamma_{\phi}\left(1+\omega_{\phi}^{1}\right)}{H_{\text {in }}(5-c)(3-c)} x_{f}^{(3-c) / 2}+\frac{\rho_{R}^{\text {in }}}{\rho_{\phi}^{\text {in }}}\left(1-\frac{2 \Gamma_{\phi}\left(1+\omega_{\phi}^{1}\right)}{H_{\text {in }}(c+3)(c+1)} x_{f}^{(c+1) / 2}\right)\right] .
$$

The dark matter relic can be obtained in terms of radiation abundance $\Omega_{R}\left(\Omega_{R} h^{2}=4.3 \times 10^{-5}\right)$ as 


$$
\Omega_{X} h^{2}=\frac{\rho_{X}\left(x_{f}\right)}{\rho_{R}\left(x_{f}\right)} \frac{T\left(x_{f}\right)}{T_{\text {now }}} \Omega_{R} h^{2}=\frac{\left\langle E_{X}\right\rangle_{f} x_{f}^{-3} n_{X}^{f}\left(x_{f}\right) x_{f}^{3}}{\rho_{R}\left(x_{f}\right)} \frac{T\left(x_{f}\right)}{T_{\text {now }}} \Omega_{R} h^{2}=0.12
$$

Inserting the expression of $n_{X}^{f} x_{f}^{3}$ [Eq. (C7)] into the above equation, one can arrive at the following equation for the dark matter abundance:

$$
\Omega_{X} h^{2} \simeq \frac{\left\langle E_{X}\right\rangle_{f} x_{f}^{-3}}{\rho_{R}\left(x_{f}\right)} \frac{T\left(x_{f}\right)}{T_{\text {now }}}\left(n_{X}^{\text {in }}+\langle\sigma v\rangle f\left(x_{f}\right)\right) \Omega_{R} h^{2} .
$$

The average energy of the single-component dark matter at the point of freeze-in can be expressed as

$$
\left\langle E_{X}\right\rangle_{f} \simeq \sqrt{M_{X}^{2}+9 T\left(x_{f}\right)^{2}} \simeq 3 T\left(x_{f}\right)\left(1+\frac{M_{X}^{2}}{18 T\left(x_{f}\right)^{2}}\right) \quad \text { (relativistic approximation) } .
$$

Therefore, connecting the above two equations ( $\mathrm{C} 10)$ and $(\mathrm{C} 11)$, one arrives at the following expression:

$$
\Omega_{X} h^{2} \simeq \frac{3 x_{f}^{-3} \rho_{R}\left(x_{f}\right)^{-1 / 2}\left(1+\frac{M_{X}^{2} \beta^{1 / 2} \rho_{R}\left(x_{f}\right)^{-1 / 2}}{18}\right)}{\beta^{1 / 2} T_{\text {now }}}\left(n_{X}^{\text {in }}+\langle\sigma v\rangle f\left(x_{f}\right)\right) \Omega_{R} h^{2} .
$$

\section{Maximum possible dark matter mass $\left(M_{X}^{\max }\right)$}

The approximate analytical expression of dark matter abundance [Eq. (C12)] indicates that the dark matter abundance increases with increasing dark matter mass. Moreover, at a particular value of the dark matter mass, the dark matter component's initial number density $\left(n_{X}^{\text {in }}\right)$ is sufficient to produce the present observed value of the dark matter abundance $\Omega_{X} h^{2}=0.12$. We define this particular value of the dark matter mass as $M_{X}^{\max }$. We can clearly see from Eq. (C12), if the mass of the dark matter $M_{X}>M_{X}^{\max }$, the abundance $\Omega_{X} h^{2}$ is always $\geq 0.12$. Therefore, the condition for the maximum possible dark matter mass can be written as

$$
\Omega_{X} h^{2} \simeq \frac{\sqrt{M_{X}^{2}+9 T\left(x_{f}\right)^{2}} x_{f}^{-3}}{\rho_{R}\left(x_{f}\right)} \frac{T\left(x_{f}\right) n_{X}^{\text {in }}}{T_{\text {now }}} \Omega_{R} h^{2}=0.12 .
$$

The outcome of this equation is the maximum possible mass $M_{X}^{\max }$, which is determined to be

$$
M_{X}^{\max }=T\left(x_{f}\right) \sqrt{\left(0.12 \frac{\beta}{n_{X}^{\text {in }}} \frac{T_{\text {now }} T\left(x_{f}\right)^{2}}{\Omega_{R} h^{2} x_{f}^{-3}}\right)^{2}-9} .
$$

[1] A. H. Guth, Phys. Rev. D 23, 347 (1981); A. D. Linde, Phys. Lett. 108B, 389 (1982); A. Albrecht and P. J. Steinhardt, Phys. Rev. Lett. 48, 1220 (1982).

[2] A. A. Starobinsky, Adv. Ser. Astrophys. Cosmol. 3, 130 (1987).

[3] Y. Akrami et al. (Planck Collaboration), Astrophys. Space Sci. 364, 69 (2019).

[4] A. R. Liddle and D. H. Lyth, Cosmological Inflation and Large Scale Structure (Cambridge University Press, Cambridge, England, 2000).

[5] A. Berera, Phys. Rev. Lett. 75, 3218 (1995).

[6] A. Berera, Phys. Rev. D 55, 3346 (1997).
[7] M. A. Amin, M. P. Hertzberg, D. I. Kaiser, and J. Karouby, Int. J. Mod. Phys. 24, 1530003 (2014).

[8] A. D Linde, Lect. Notes Phys. 738, 1 (2008).

[9] B. A. Bassett, S. Tsujikawa, and D. Wands, Mod. Phys. 78, 537 (2006).

[10] J. Martin, T. Papanikolaou, L. Pinol, and V. Vennin, J. Cosmol. Astropart. Phys. 05 (2020) 003.

[11] J. Martin and C. Ringeval, Phys. Rev. D 82, 023511 (2010); J. Martin, C. Ringeval, and V. Vennin, Phys. Rev. Lett. 114, 081302 (2015); L. Dai, M. Kamionkowski, and J. Wang, Phys. Rev. Lett. 113, 041302 (2014).

[12] M. Drewes, J. Cosmol. Astropart. Phys. 03 (2016) 013. 
[13] I. Dalianis and Y. Watanabe, J. High Energy Phys. 02 (2018) 118.

[14] M. Drewes, arXiv:1903.09599.

[15] E. W. Kolb, A. D. Linde, and A. Riotto, Phys. Rev. Lett. 77, 4290 (1996).

[16] A. Dolgov, K. Freese, R. Rangarajan, and M. Srednicki, Phys. Rev. D 56, 6155 (1997).

[17] A. Riotto and M. Trodden, Annu. Rev. Nucl. Part. Sci. 49, 35 (1999).

[18] J. Garcia-Bellido, D. Y. Grigoriev, A. Kusenko, and M. E. Shaposhnikov, Phys. Rev. D 60, 123504 (1999).

[19] R. Allahverdi, B. A. Campbell, and J. R. Ellis, Nucl. Phys. B579, 355 (2000).

[20] S. Davidson, M. Losada, and A. Riotto, Phys. Rev. Lett. 84, 4284 (2000).

[21] A. Megevand, Phys. Rev. D 64, 027303 (2001).

[22] S. A. Gorbunov et al. (NEWSdm Collaboration), Phys. At. Nucl. 83, 83 (2020).

[23] M. S. Turner and F. Wilczek, Phys. Rev. Lett. 65, 3080 (1990).

[24] A. Kosowsky, M. S. Turner, and R. Watkins, Phys. Rev. Lett. 69, 2026 (1992).

[25] F. Finelli and R. H. Brandenberger, Phys. Rev. Lett. 82, 1362 (1999).

[26] R. Easther, J. T. Giblin, Jr., and E. A. Lim, Phys. Rev. Lett. 99, 221301 (2007).

[27] R. Easther and E. A. Lim, J. Cosmol. Astropart. Phys. 04 (2006) 010.

[28] J. Garcia-Bellido and D. G. Figueroa, Phys. Rev. Lett. 98, 061302 (2007).

[29] J. Garcia-Bellido, D. G. Figueroa, and A. Sastre, Phys. Rev. D 77, 043517 (2008).

[30] J. F. Dufaux, A. Bergman, G. N. Felder, L. Kofman, and J. Uzan, Phys. Rev. D 76, 123517 (2007).

[31] N. Bernal and F. Hajkarim, Phys. Rev. D 100, 063502 (2019).

[32] K. D. Lozanov and M. A. Amin, Phys. Rev. D 99, 123504 (2019).

[33] M. Yu. Khlopov and A. D. Linde, Phys. Lett. 138B, 265 (1984); V. Domcke and J. Heisig, Phys. Rev. D 92, 103515 (2015).

[34] P. Saha, S. Anand, and L. Sriramkumar, arXiv:2005 .01874 .

[35] D. Maity and P. Saha, Phys. Rev. D 98, 103525 (2018).

[36] D. Maity and P. Saha, Phys. Dark Universe 25, 100317 (2019).

[37] D. Maity and P. Saha, J. Cosmol. Astropart. Phys. 07 (2019) 018.

[38] L. Kofman, A. D. Linde, and A. A. Starobinsky, Phys. Rev. Lett. 73, 3195 (1994); Phys. Rev. D 56, 3258 (1997); Y. Shtanov, J. H. Traschen, and R. H. Brandenberger, Phys. Rev. D 51, 5438 (1995).

[39] K. Freese, E. I. Sfakianakis, P. Stengel, and L. Visinelli, J. Cosmol. Astropart. Phys. 05 (2018) 067.

[40] R. Easther, R. Flauger, and J. B. Gilmore, J. Cosmol. Astropart. Phys. 04 (2011) 027.

[41] D. G. Figueroa and F. Torrenti, J. Cosmol. Astropart. Phys. 02 (2017) 001.

[42] S. Antusch, D. G. Figueroa, K. Marschall, and F. Torrenti, arXiv:2005.07563.
[43] K. D. Lozanov and M. A. Amin, Phys. Rev. Lett. 119, 061301 (2017).

[44] K. D. Lozanov and M. A. Amin, Phys. Rev. D 97, 023533 (2018).

[45] P. A. R. Ade et al. (Planck Collaboration), Astron. Astrophys. 594, A20 (2016); P. A. R. Ade et al. (Keck Array and BICEP2 Collaborations), Phys. Rev. Lett. 116, 031302 (2016).

[46] G. F. Giudice, E. W. Kolb, and A. Riotto, Phys. Rev. D 64, 023508 (2001).

[47] L. Amendola et al. (Euclid Theory Working Group Collaboration), Living Rev. Relativity 16, 6 (2013).

[48] P. Andre et al. (PRISM Collaboration), arXiv:1306.2259.

[49] V.C. Rubin, High Energy Astrophysics (Gordon and Breach, New York, 1967), Vol. 1, pp. 133-152.

[50] V. C. Rubin and W. Ford, Jr., Astrophys. J. 159, 379 (1970).

[51] W. Hu and S. Dodelson, Annu. Rev. Astron. Astrophys. 40, 171 (2002).

[52] G. Bertone, D. Hooper, and J. Silk, Phys. Rep. 405, 279 (2005).

[53] L. F. Abbott, E. Farhi, and M. B. Wise, Phys. Lett. 117B, 29 (1982); A. Albrecht, P. J. Steinhardt, M. S. Turner, and F. Wilczek, Phys. Rev. Lett. 48, 1437 (1982).

[54] J. Garcia-Bellido, D. G. Figueroa, and J. Rubio, Phys. Rev. D 79, 063531 (2009).

[55] J. Repond and J. Rubio, J. Cosmol. Astropart. Phys. 07 (2016) 043.

[56] M. E. Peskin and D. V. Schroeder, An Introduction to Quantum Field Theory (Addison-Wesley, Reading, USA, 1995).

[57] M. Drewes, J. U. Kang, and U. R. Mun, J. High Energy Phys. 11 (2017) 072.

[58] C. Patrignani et al. (Particle Data Group), Chin. Phys. C 40, 100001 (2016).

[59] P. B. Greene and L. Kofman, Phys. Lett. B 448, 6 (1999).

[60] D. I. Podolsky, G. N. Felder, L. Kofman, and M. Peloso, Phys. Rev. D 73, 023501 (2006).

[61] Daniel J. H. Chung, Edward W. Kolb, and Antonio Riotto, Phys. Rev. D 60, 063504 (1999).

[62] R. Allahverdi, R. Brandenberger, F. Cyr-Racine, and A. Mazumdar, Annu. Rev. Nucl. Part. Sci. 60, 27 (2010).

[63] M. A. Amin, M. P. Hertzberg, D. I. Kaiser, and J. Karouby, Int. J. Mod. Phys. D 24, 1530003 (2014).

[64] G. N. Felder and I. Tkachev, Comput. Phys. Commun. 178, 929 (2008).

[65] G. N. Felder, Comput. Phys. Commun. 179, 604 (2008).

[66] V. Mukhanov, Physical Foundations of Cosmology (Cambridge University Press, Cambridge, England, 2005).

[67] A. D. Linde, Phys. Lett. 129B, 177 (1983).

[68] M. Kawasaki, K. Kohri, and N. Sugiyama, Phys. Rev. Lett. 82, 4168 (1999).

[69] M. Kawasaki, K. Kohri, and N. Sugiyama, Phys. Rev. D 62, 023506 (2000).

[70] G. Steigman, Annu. Rev. Nucl. Part. Sci. 57, 463 (2007).

[71] B. D. Fields, P. Molaro, and S. Sarkar, Chin. Phys. C 38, 339 (2014).

[72] K. Freese, J. A. Frieman, and A. V. Olinto, Phys. Rev. Lett. 65, 3233 (1990). 
[73] K. Freese and W. H. Kinney, J. Cosmol. Astropart. Phys. 03 (2015) 044.

[74] M. Gerbino, K. Freese, S. Vagnozzi, M. Lattanzi, O. Mena, E. Giusarma, and S. Ho, Phys. Rev. D 95, 043512 (2017).

[75] R. Kallosh and A. Linde, J. Cosmol. Astropart. Phys. 07 (2013) 002; R. Kallosh, A. Linde, and D. Roest, J. High Energy Phys. 11 (2013) 198.

[76] F. L. Bezrukov and M. Shaposhnikov, Phys. Lett. B 659, 703 (2008).

[77] D. Maity and P. Saha, Classical Quantum Gravity 36, 045010 (2019).

[78] E. Komatsu et al. (WMAP Collaboration), Astrophys. J. Suppl. Ser. 192, 18 (2011).

[79] D. Maity, Nucl. Phys. B919, 560 (2017).

[80] G. F. Giudice, E. W. Kolb, and A. Riotto, Phys. Rev. D 64, 023508 (2001).

[81] N. Jarosik et al. (WMAP Collaboration), Astrophys. J. Suppl. Ser. 192, 14 (2011).

[82] N. Aghanim et al. (Planck Collaboration), Astron. Astrophys. 641, A6 (2020).

[83] T. Tenkanen, J. High Energy Phys. 09 (2016) 049.

[84] M. Heikinheimo, T. Tenkanen, K. Tuominen, and V. Vaskonen, Proc. Sci. ICHEP2016 (2016) 825 [arXiv:1611 .04951].

[85] N. Bernal, M. Heikinheimo, T. Tenkanen, K. Tuominen, and V. Vaskonen, Int. J. Mod. Phys. A 32, 1730023 (2017).

[86] L. J. Hall, K. Jedamzik, J. March-Russell, and S. M. West, J. High Energy Phys. 03 (2010) 080.

[87] X. Chu, Y. Mambrini, J. Quevillon, and B. Zaldivar, J. Cosmol. Astropart. Phys. 01 (2014) 034.

[88] M. Blennow, E. Fernandez-Martinez, and B. Zaldivar, J. Cosmol. Astropart. Phys. 01 (2014) 003.

[89] F. Elahi, C. Kolda, and J. Unwin, J. High Energy Phys. 03 (2015) 048.

[90] Y. Mambrini, N. Nagata, K. A. Olive, J. Quevillon, and J. Zheng, Phys. Rev. D 91, 095010 (2015).

[91] N. Nagata, K. A. Olive, and J. Zheng, J. High Energy Phys. 10 (2015) 193.

[92] S. Chen and Z. Kang, J. Cosmol. Astropart. Phys. 05 (2018) 036.

[93] N. Bernal, M. Dutra, Y. Mambrini, K. Olive, M. Peloso, and M. Pierre, Phys. Rev. D 97, 115020 (2018).

[94] N. Bernal, C. Cosme, T. Tenkanen, and V. Vaskonen, Eur. Phys. J. C 79, 30 (2019).

[95] Y. Mambrini, K. A. Olive, J. Quevillon, and B. Zaldivar, Phys. Rev. Lett. 110, 241306 (2013).

[96] M. A. Garcia and M. A. Amin, Phys. Rev. D 98, 103504 (2018).

[97] R. Allahverdi, K. Dutta, and A. Maharana, J. Cosmol. Astropart. Phys. 10 (2018) 038.
[98] A. Ahmed, B. Grzadkowski, and A. Socha, J. High Energy Phys. 08 (2020) 059.

[99] P. Arias, N. Bernal, A. Herrera, and C. Maldonado, J. Cosmol. Astropart. Phys. 10 (2019) 047.

[100] N. Bernal, X. Chu, S. Kulkarni, and J. Pradler, Phys. Rev. D 101, 055044 (2020).

[101] N. Bernal, F. Elahi, C. Maldonado, and J. Unwin, J. Cosmol. Astropart. Phys. 11 (2019) 026.

[102] M. Dutra, M. Lindner, S. Profumo, F. S. Queiroz, W. Rodejohann, and C. Siqueira, J. Cosmol. Astropart. Phys. 03 (2018) 037.

[103] L. Heurtier and F. Huang, Phys. Rev. D 100, 043507 (2019).

[104] C. Miller, A. L. Erickcek, and R. Murgia, Phys. Rev. D 100, 123520 (2019).

[105] L. Heurtier, J. High Energy Phys. 12 (2017) 072.

[106] K. Griest and M. Kamionkowski, Phys. Rev. Lett. 64, 615 (1990).

[107] N. Fornengo, A. Riotto, and S. Scopel, Phys. Rev. D 67, 023514 (2003).

[108] F. L. Bezrukov and M. Shaposhnikov, Phys. Lett. B 659, 703 (2008).

[109] P. A. R. Ade et al. (Planck Collaboration), Astron. Astrophys. 571, A22 (2014); Astron. Astrophys. 594, A20 (2016).

[110] P. Adshead, R. Easther, J. Pritchard, and A. Loeb, J. Cosmol. Astropart. Phys. 02 (2011) 021; R. Easther and H. V. Peiris, Phys. Rev. D 85, 103533 (2012); J. B. Munoz and M. Kamionkowski, Phys. Rev. D 91, 043521 (2015); J. L. Cook, E. Dimastrogiovanni, D. A. Easson, and L. M. Krauss, J. Cosmol. Astropart. Phys. 04 (2015) 047.

[111] J. Ellis, M. A. G. Garcia, D. V. Nanopoulos, and K. A. Olive, J. Cosmol. Astropart. Phys. 07 (2015) 050.

[112] W. T. Emond, P. Millington, and P. M. Saffin, J. Cosmol. Astropart. Phys. 09 (2018) 041.

[113] E. W. Kolb and M.S. Turner, The Early Universe (Westview Press, 1990).

[114] P. Gondolo and G. Gelmini, Nucl. Phys. B360, 145 (1991).

[115] M. Hindmarsh and O. Philipsen, Phys. Rev. D 71, 087302 (2005).

[116] M. Laine and Y. Schroder, Phys. Rev. D 73, 085009 (2006).

[117] M. Drees, F. Hajkarim, and E. R. Schmitz, J. Cosmol. Astropart. Phys. 06 (2015) 025.

[118] M. Drees and F. Hajkarim, J. Cosmol. Astropart. Phys. 02 (2018) 057.

[119] M. Srednicki, R. Watkins, and K. A. Olive, Nucl. Phys. B310, 693 (1988). 